\title{
Multi-color fluorescent DNA analysis in an integrated optofluidic lab on a chip
}

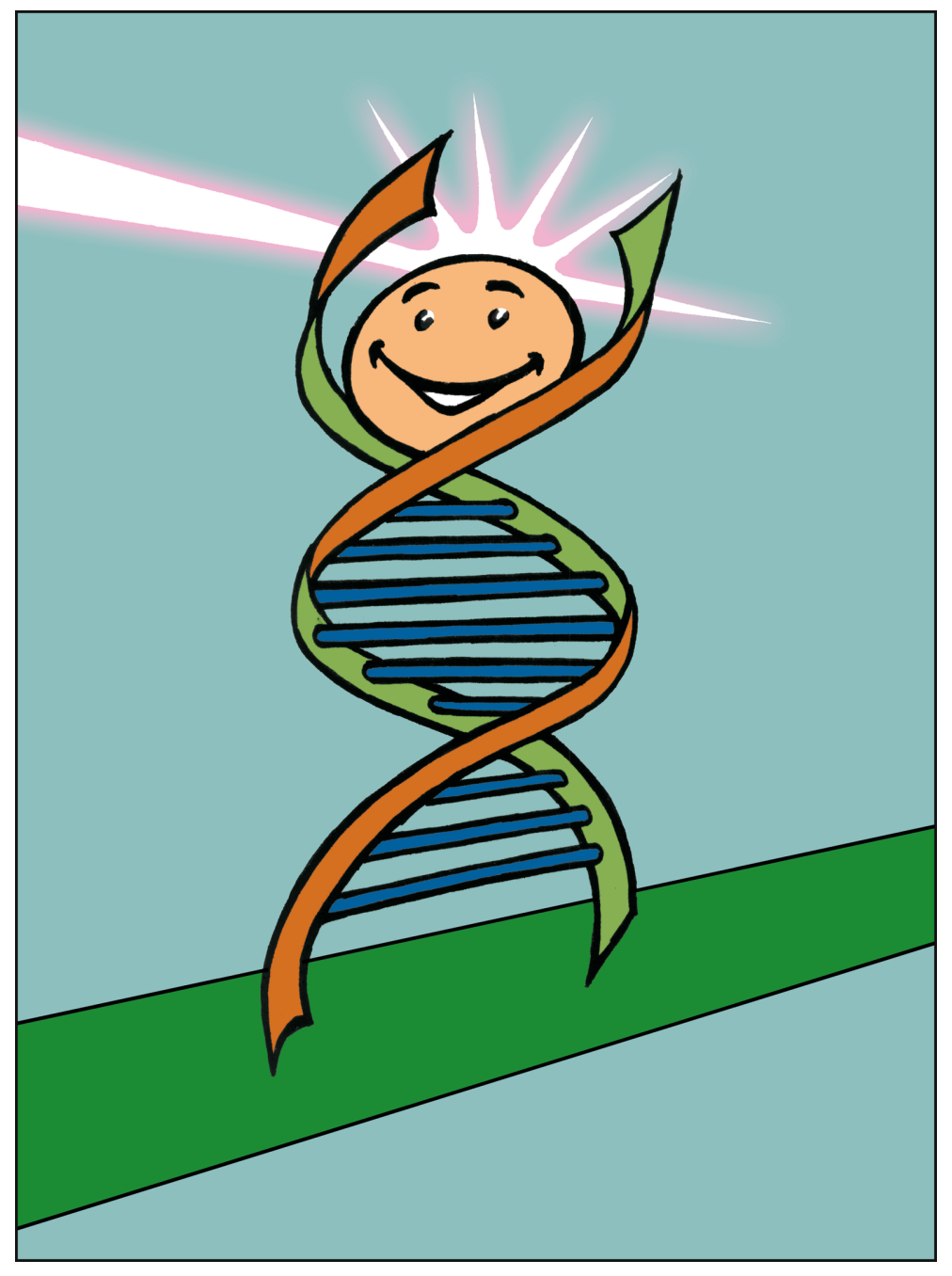

Chaitanya Dongre 


\section{Multi-color fluorescent DNA analysis in an integrated optofluidic lab on a chip}

Chaitanya Dongre 


\section{Graduation committee:}

Chairman and secretary:

Prof. Dr. A.J. Mouthaan

University of Twente

\section{Promoter:}

Prof. Dr. M. Pollnau

University of Twente

\section{Assistant promoter:}

Dr. H.J.W.M. Hoekstra University of Twente

\section{Members:}

Prof. Dr. C. Depeursinge

Prof. Dr. V. Subramaniam

EPFL (Swiss Federal Institute of Technology, Lausanne)

Prof. Dr. J.G.E. Gardeniers

University of Twente

Dr. G.A.J. Besselink

University of Twente

CapiliX BV

The research described in this thesis has been carried out at the "Integrated Optical MicroSystems" group (IOMS) of the MESA+ Institute for Nanotechnology at the University of Twente, Enschede, The Netherlands.

This work was financially supported by the EU Project HIBISCUS (Hybrid integrated biophotonic sensors created by ultrafast laser systems), within the European Union's sixth framework program (FP6), through the contract IST-2005034562 .

Front cover: Fluorescence excitation of a labeled DNA molecule, an artist's impression (designed by Ashok Dongre, Mumbai, India).

Back cover: Ultrafast laser optofluidic integration, waveguide excitation of flowing fluorescent molecules, and multi-color fluorescent DNA analysis, for "life enriched by light" (original Sanskrit text by Pournima Dongre, Mumbai, India).

ISBN: 978-90-365-3070-5

Printed by Wöhrmann Print Service, Zutphen, The Netherlands.

(C) 2010 by Chaitanya Dongre, Enschede, The Netherlands. 


\title{
MULTI-COLOR FLUORESCENT DNA ANALYSIS IN AN INTEGRATED OPTOFLUIDIC LAB ON A CHIP
}

\section{DISSERTATION}

\author{
to obtain \\ the degree of doctor at the University of Twente, \\ on the authority of the rector magnificus, \\ prof. dr. H. Brinksma,
}

on account of the decision of the graduation committee, to be publicly defended on Wednesday the $25^{\text {th }}$ of August 2010 at $13: 15$

by

Chaitanya Dongre

born on the $11^{\text {th }}$ of November 1983

in Mumbai, India 
This dissertation is approved by:

The promoter: Prof. Dr. M. Pollnau

The assistant promoter: Dr. H.J.W.M. Hoekstra 


\section{Contents}

$\begin{array}{ll}\text { Abstract } & 7\end{array}$

$\begin{array}{lr}\text { 1. Introduction } & 8\end{array}$

1.1 Biomolecule analysis by electrophoresis separation 8

$\begin{array}{ll}1.2 \text { Lab-on-a-chip } & 10\end{array}$

1.3 Integrated optofluidics 11

1.4 Outline of the thesis $\quad 14$

2. Optofluidic integration in a lab-on-a-chip 16

2.1 Introduction $\quad 16$

2.2 Integrated optofluidic sample fabrication $\quad 16$

2.2.1 Microfluidic chip fabrication $\quad 16$

2.2.2 Optical post-processing by femtosecond-laser waveguide writing 20

2.3 Characterization of the integrated waveguides 23

2.3.1 Near-field imaging $\quad 24$

2.3.2 Refractive index profile $\quad 25$

2.3.3 Propagation loss 28

2.4 Optofluidic characterization of the lab-on-a-chip 29

2.4.1 Sensing of static events $\quad 30$

2.4.2 Sensing of dynamic flow events $\quad 35$

$\begin{array}{ll}2.5 \text { Summary } & 39\end{array}$

3. Fluorescence monitoring of on-chip DNA sorting 40

3.1 Introduction $\quad 40$

3.2 Optimization of microfluidic parameters $\quad 40$

3.2.1 The microfluidic channels 41

3.2.2 Suppressing electro-osmotic flow $\quad 42$

3.2.3 Molecular sieving $\quad 45$

3.3 Electrophoretic DNA sorting $\quad 47$

3.3.1 Experimental protocol $\quad 47$

3.3.2 Experimental results and analysis $\quad 48$

3.3.3 Capillary electrophoresis separation resolution $\quad 52$

$\begin{array}{ll}3.4 \text { Summary } & 55\end{array}$ 
4.1 Introduction $\quad 57$

4.2 Optimization of the experimental setup 58

4.2.1 Integrated optical excitation as opposed to Hg-lamp 60

4.2.2 Microfluidic sample stacking 63

4.3 All-numerical lock-in amplification 65

4.4 Summary 72

5. Multi-color fluorescent DNA analysis 73

5.1 Introduction 73

5.2 Dual-point fluorescence sensing $\quad 74$

5.2.1 Materials and methods $\quad 76$

5.2.2 Experimental results and discussion $\quad 76$

5.3 Modulation-encoding and Fourier-analytical decoding $\quad 79$

$\begin{array}{ll}\text { 5.3.1 Description of the principle } & 79\end{array}$

5.3.2 Experimental proof of principle $\quad 81$

5.4 Application of modulation-frequency encoding to multiplex genetic diagnostics $\quad 83$

5.4.1 Multiplex ligation-dependent probe amplification $\quad 83$

5.4.2 Experimental protocol 86

5.4.3 Fluorescence excitation and detection $\quad 86$

$\begin{array}{ll}\text { 5.4.4 Experimental results } & 87\end{array}$

5.4.5 Broad potential of the method 90

$\begin{array}{ll}5.5 \text { Summary } & 92\end{array}$

6. Conclusions and outlook 93

List of abbreviations $\quad 96$

$\begin{array}{ll}\text { References } & 97\end{array}$

List of publications $\quad 116$

$\begin{array}{ll}\text { Samenvatting } & 123\end{array}$

$\begin{array}{ll}\text { Acknowledgements } & 124\end{array}$ 


\section{Abstract}

Sorting and sizing of DNA molecules within the human genome project has enabled the genetic mapping of various illnesses. Furthermore by employing tiny lab-on-a-chip device, integrated DNA sequencing and genetic diagnostics have become feasible. We present the combination of capillary electrophoresis with laser-induced fluorescence for optofluidic integration toward an on-chip bio-analysis tool. Integrated optical fluorescence excitation allows for a high spatial resolution $(12 \mu \mathrm{m})$ in the electrophoretic separation channel, and can lead to a further 20 -fold enhancement as soon as improved microfluidic protocols become available. We demonstrate accurate sizing (with $>99 \%$ sizing accuracy) and highly sensitive (LOD $=220$ femtomolar, corresponding to merely 6 molecules in the excitation volume) fluorescence detection of double-stranded DNA molecules by integratedwaveguide laser excitation. Subsequently, we introduced a principle of parallel optical processing to this optofluidic lab on a chip. In this approach, different sets of exclusively color-labeled DNA fragments - otherwise rendered indistinguishable by their spatial (in the microchip CE separation channel) and temporal (in the consequent electropherogram) coincidence are traced back to their origin by modulation-frequency-encoded multiwavelength laser excitation, fluorescence detection with a color-blind photomultiplier, and Fourier-analytical decoding. As a proof of principle, fragments obtained by multiplex ligation-dependent probe amplification from independent human genomic segments, associated with genetic predispositions to breast cancer and anemia, are simultaneously analyzed. The techniques described in this thesis for multiple, yet unambiguous optical identification of biomolecules will potentially open new horizons for "enlightened" lab-on-a-chip devices in the future. 


\section{Introduction}

A biochemical lab on a chip (LOC) [Manz, 1990] [Jakeway, 2000] [Reyes, 2002] [Auroux, 2002] squeezes the functionalities of a biological/chemical laboratory onto a single substrate through a network of microfluidic (MF) channels, reservoirs, valves, pumps and sensors. Its advantages are high sensitivity, speed of analysis, low sample consumption and measurement automation and standardization. This concept promises dramatic advances both in basic research and in clinical applications, e.g. as a low-cost diagnostic tool. Within the project HIBISCUS [Hibiscus, web] it was proposed to demonstrate the combination of two existing technologies, namely the clean-room based fabrication of MF chips, and the writing of optical waveguides (WGs) with high intensity femtosecond (fs-) laser pulses, providing an platform for the fabrication of LOCs with photonic functionalities. Such technology enables fs-laser written optical WGs on a standard LOC, in different configurations with respect to the MF channels, e.g. three-dimensional (3D) Mach-Zehnder interferometers exploiting the unique 3D capabilities of the fs-laser writing technique [Crespi, 2010], or coplanar WGs intersecting the MF channel for fluorescence excitation [Martinez Vazquez, 2009]. This unique integration of photonics and microfluidics, resulting from the inscription of optical WGs on the LOCs, is envisioned to enable a wealth of novel biosensing functionalities. In this thesis, we concentrated on a prototypical device, namely a DNA assay based on capillary electrophoresis (CE) separation for the multiplex detection of genetic abnormalities by means of multi-color fluorescence sensing. In this first chapter of the thesis, we will introduce the field of fluorescent DNA analysis in CE-based chips, describing the state of the art and the broad relevance of the field.

\subsection{Biomolecular analysis by electrophoretic separation}

One of the most powerful methods for the analysis of biomolecules is CE, in which electrically charged or polarized molecules are separated in a fluidic channel due to their different electrophoretic mobilities under an applied electric field, where the mobilities in turn act as pointers to other physical characteristics of the analyte molecules, e.g. the size in base-pairs (bp) in the case of negatively charged DNA molecules. This technique is normally performed in a glass capillary filled with a buffer solution or a sieving gel matrix, e.g. agarose gel [Brody, 2004]; CE is however particularly suited for 
on-chip integration [Harrison, 1993] [Landers, 2003], since electrokinetic flow can be used to move and mix liquids, thus avoiding the need to integrate pumps and valves [Bruin, 2000]. Microchip CE (MCE) is particularly promising for clinical applications [Zhang, 2003], since it allows one to perform genetic tests to diagnose a variety of diseases, both exogenous (such as bacterial or viral infections) and endogenous (detection of mutated DNA sequences related to cancer or genetically inherited diseases). The sorting and sizing of DNA molecules within the human genome project [Lander, 2001] has been enabled largely by CE separation and analysis [Slater, 2003]. The human genome project has also lead to the genetic mapping of various human illnesses [Altshuler, 2008]. By employing the understanding of DNA separation by MF CE, on-chip integration of DNA sequencing [Fredlake, 2008] [Eid, 2009] [Pile, 2009] [Pacific biosciences, web] as well as genetic diagnostics [Lagally, 2004] [Easley, 2006] have become feasible.

In this thesis we focus on a specific $\mathrm{CE}$ approach, making use of MF channels whose walls are coated with epoxy-poly-dimethyl acrylamide (EPDMA) [Cretich, 2003] - in order to suppress electroosmotic flow (EOF) - in a fused silica LOC, where a sieving gel matrix is used in order to further enhance the CE separation of fluorescently intercalated or end-labeled DNA molecules. Monitoring CE separation of fluorescently labeled DNA molecules corresponds essentially to the analysis of the fluorescence intensity at a specific point (e.g. where the MF channel and the optical WG intersect each other, or where the light beam from a $\mathrm{Hg}$ arc lamp is focused onto the MF channel) along the CE separation channel, as a function of time. This leads to an electropherogram (Fig. 1.1) where the fluorescence peaks correspond to the CE-separated, specifically sized DNA molecules present in the original analyte sample mixture introduced to the LOC.

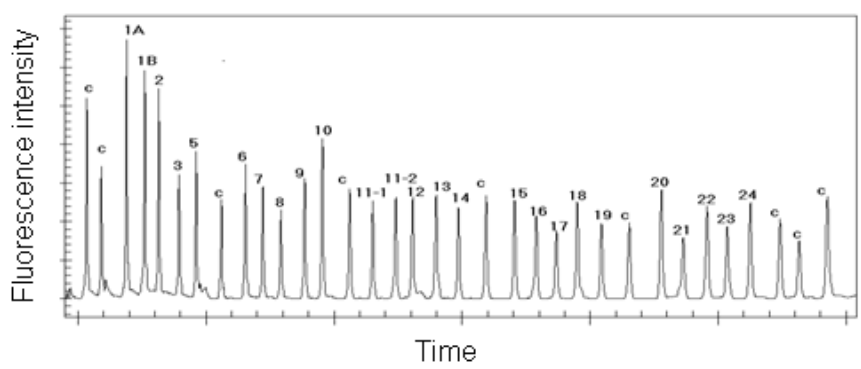

Fig. 1.1 A typical electropherogram obtained from a conventional DNA CE separation instrument (courtesy of Zebra Bioscience BV [Zebra, web]) 
The traditional, bulk capillary based techniques provide a very high separation resolution, as will be pointed out in detail in chapter 3, section 3.3.3. However, they typically have longer analysis times and bulky instrumentation. This point has been addressed by the development of microchip CE. Also, the bulk optical detection schemes making use of freespace excitation/detection optics cannot be easily integrated in a compact device. The focus of this thesis will be essentially to demonstrate the value addition of integrated optics to such CE-based DNA analysis on LOCs, e.g. by showing potential enhancement of the separation resolution, enhancement of the sensitivity - enabling us to detect low concentrations of permanently, exclusively end-labeled DNA molecules, etc. This functionality finally enabled the implementation of multiplex (multi-color) fluorescent analysis of DNA probe samples from two different genes, corresponding to two different illnesses, analyzed unambiguously during a single $\mathrm{CE}$ experiment.

\subsection{Lab on a chip}

The LOC concept is currently undergoing fast development and promises dramatic advances in its application areas encompassing basic science (genomics and proteomics) to chemical synthesis and drug developments [de Mello, 2006], high-throughput medical and biochemical analysis [Yager, 2006], and environmental monitoring and detection of chemical and biological threats. In order to be successful, the LOC devices must be compact, mass producible, flexible in their application, robust, and cost efficient. LOCs are mainly fabricated in polymer, glass, or silicon substrates. Polymers are quickly becoming the most common choice due to their low cost and the simplicity of microchannel fabrication by molding or embossing. Nevertheless, glass is still preferred in several applications [Dishinger, 2007] as it is chemically inert, stable in time, hydrophilic, nonporous and optically transparent. In particular, the choice of fused silica as the basic material adds to the previous advantages a very high optical transparency even down to the UV range and a very low intrinsic fluorescence. In addition, well established microfabrication processes, based on photolithography and etching, are available for glass.

The reduced analysis volumes in LOC systems lead to low signal levels, since fewer molecules are present in the detection region. Different detection strategies are possible, but among them the most popular is laser induced fluorescence (LIF) [Johnson, 2004] which, being a background-free technique, allows the measurement of low analyte concentrations in the picomolar range, e.g. 250 pM [Hubner, 2001], 500 pM [Bliss, 2007], typically making use of visible or even near-infrared [Soper, 1996] fluorescent dye labels attached to the analyte molecules. In traditional set- 
ups used in conjunction with LOCs, both excitation and detection are performed using bulk optical equipment, such as mirrors, lenses and microscope objectives in confocal setups [Dittrich, 2003] [Campbell, 2004], to focus the excitation light into a tiny measurement volume and to collect the resulting fluorescence. Such schemes can provide high sensitivity, with the vision - backed by experimental demonstrations - of going down to interrogating at the single-molecule level [Craighead, 2006] [Dittrich, 2005]; however, they require accurate mechanical alignment of the optics to the MF channels, and are sensitive to mechanical vibrations and drifts. The need to use a miniaturized LOC system in combination with a massive benchtop instrument such as an optical microscope, frustrates many of the LOC advantages; in particular it strongly limits device portability and prevents infield or point-of-care applications. Much of the commercial success of the LOC concept will critically depend on the ability to successfully integrate optical detection schemes. Such integration promises a significant reduction in size, cost and complexity of the system.

Fluorescent marker molecules can be chemically attached to a specific sample molecule of interest so that fluorescence sensing can be used for quantitative analysis. In recent years, some preliminary efforts have been devoted to integrate optical WG systems on MF LOCs [Ruano, 2000] [Friis, 2001]. Use of optical WGs has many clear advantages with respect to standard free-space detection systems, in terms of alignment precision, compactness and portability. In the next section 1.3, we will discuss in detail the various approaches that are available and that have been explored for optofluidic integration in LOCs.

\subsection{Integrated Optofluidics}

Several efforts have been performed in order to integrate micro-optical components in MF LOCs to perform on-chip optical detection [Verpoorte, 2003] [Goetz, 2007] [Hunt, 2008] [Myers, 2008]. In addition to the integration of optical WGs, also other techniques have been explored for optofluidic integration in LOCs, e.g. the use of the MF channel itself as a liquid core optofluidic WG [Mach, 2002] [Wolfe, 2005] [Yin, 2006] or even a liquid core optofluidic laser [Vezenov, 2005] [Li, 2006], silicon-based optoelectronic transducer elements [Misiakos, 2004], optical fibers for fluorescence excitation [Lin, 2004], integration of lasers for fluorescence excitation [Cran-McGreehin, 2006], optofluidic lenses for fluorescence collection [Song, 2009], photodetectors for fluorescence detection [Namasivayam, 2004], etc..

Integrated optical WGs allow to confine and transport light in the chip, directing it to a small volume of the MF channel and collecting the 
emitted/transmitted light, as has recently been applied to monitor on-chip DNA sequencing using zero-mode WG sensors [Levene, 2003] [Eid, 2009] in a now commercialized DNA sequencer [Pacific biosciences, web]. The integration of optical WGs or other photonic components with MF channels typically needs additional processing steps. Depending on the substrate of choice, different WG fabrication processes are possible. Several such approaches are reported in the literature and include silica on silicon [Mogensen, 2001] [Friis, 2001] [Hubner, 2001], ion-exchange in soda-lime glasses [Mazurczyk, 2006] [Vieillard, 2007], photolithography in polymers [Kuswandi, 2007] [Mogensen, 2003] and liquid core WGs [Bliss, 2007] [Bliss, 2008] [Yin, 2006] [Yin, 2007].

In the silica-on-silicon technology WGs are fabricated on the silicon substrate by deposition of core and cladding silica layers with the refractiveindex contrast being controlled e.g. by means of varying the dopants and their concentrations, patterned by reactive-ion etching. A further photolithographic step is then performed to etch the microchannels, which are then hermetically sealed with a cover glass. These devices were tested both for absorption [Mogensen, 2001] and fluorescence [Hubner, 2001] detection. These WGs are multimode and have propagation losses of typically $1 \mathrm{~dB} / \mathrm{cm}$ at $530 \mathrm{~nm}$. Detection by UV absorption measurements of a beta-blocking agent (propranolol) used to treat hypertension was demonstrated, with a limit of detection (LOD) of $13 \mu \mathrm{M}$ [Mogensen, 2001]. On the other hand, exploiting fluorescence, fluorescein was detected with a LOD of $250 \mathrm{pM}$ [Hubner, 2001].

In the case of LOC in soda-lime glass, first the WGs for fluorescence excitation are fabricated by the ion-exchange technique and then the microchannels are produced, on the same substrate, by photolithography and wet etching [Mazurczyk, 2006] [Vieillard, 2007]. Using a Rhodamine 6G solution a minimum LOD of $500 \mathrm{pM}$ was obtained and different protein mixtures were separated and detected by LIF [Mazurczyk, 2006].

Several research groups used polymeric materials to combine MF channels and optical WGs. MF channels and WGs were fabricated in a single step by standard photolithography on a negative photoresist (SU-8) film, sandwiched between polymer or glass slabs [Mogensen, 2003]. The WGs are multimode and have propagation losses of $1.4 \mathrm{~dB} / \mathrm{cm}$ at $633 \mathrm{~nm}$. The device was designed to perform absorption measurements and was tested at $633 \mathrm{~nm}$ on a bromothymol blue dye solution filling the channels. It provided a LOD of $15 \mu \mathrm{M}$.

In a recent approach using liquid-core WGs [Bliss, 2007] [Bliss, 2008] the LOC devices were fabricated by soft lithography on poly(dimethylsiloxane) (PDMS) and WGs were created in dedicated channels by the addition of a liquid PDMS prepolymer of higher refractive 
index. WG propagation losses are $1.8 \mathrm{~dB} / \mathrm{cm}$ at $532 \mathrm{~nm}$ and $1.0 \mathrm{~dB} / \mathrm{cm}$ at $633 \mathrm{~nm}$. The device, designed for LIF applications and tested in the separation of a BK virus polymerase chain reaction product, providing a signal-to-noise ratio $(570 \pm 30)$ for the specific sample in the same order of magnitude as the commercially available confocal-based systems $(330 \pm 30)$.

A device based on planar networks of intersecting solid- and liquid-core WGs was fabricated [Yin, 2006] [Yin, 2007], where the fluorescent molecules are inside the "liquid" core and both types of WGs are used to deliver and collect the excitation and fluorescence light. Fluorescently labeled liposomes are used to demonstrate single molecule detection by fluorescence correlation spectroscopy.

From these and other examples it can be concluded that the fabrication of optical WGs integrated with the MF channels is not always a straightforward process. Since the production of optical WGs typically requires a localized increase of the refractive index in the device volume, whatever technology is chosen, it strongly affects the fabrication procedure of the MF part of the chip. In summary, the most widely used optical WG fabrication techniques around the start of this work were among others ion exchange, chemical vapor deposition followed by reactive ion etching (the so-called silica-onsilicon approach) [Kuswandi, 2007]. All these approaches, while being adequate for many applications (e.g. large-scale manufacturing of telecom components), can nevertheless be improved on in a number of aspects, when applied to LOCs, e.g. the fact that silica-on-silicon approach creates non-flat glass surfaces, making the sealing of the MF channels problematic, must be addressed. This is one reason why the integration of optical WGs within MF LOCs has so far been limited.

The technique for writing WGs with a fs laser emerged in the past two decades [Davis, 1996] [Itoh, 2006] [Gattass, 2008] [Osellame, 2006] [Eaton, 2006]. Within the project HIBISCUS [Hibiscus, web] and within the context of this thesis, the technique for direct writing of WGs and photonic circuits in transparent glass substrates by focused fs pulses was performed by the group of Prof. G. Cerullo at the Politecnico di Milano [Martinez Vazquez, 2009]. The ultrashort pulse duration $\left(\left(10^{-13} \mathrm{~s}\right)\right.$ leads to very high peak powers $\left((10 \mathrm{MW})\right.$ and intensities $\left(\left(10^{14} \mathrm{~W} / \mathrm{cm}^{2}\right)\right.$, when focused by a microscope objective, even for low average powers (tens of $\mathrm{mW}$ ). Due to such high intensities, the interaction of the laser pulse with the material becomes strongly nonlinear; in particular, a very selective absorption (due to a combination of multi-photon absorption and avalanche ionization processes) occurs only in a small volume around the focus, where the intensity is the highest, allowing highly spatially-selective energy deposition. The hot electron plasma induces high temperatures and pressures that give rise to structural modifications such as densification and color-center creation 
[Gattass, 2008]. The combination of all these effects leads, under suitable conditions and in a suitable material, to a local increase of the refractive index, which can be exploited, by moving the laser focus inside the substrate, in order to produce three-dimensional light-guiding structures.

Compared with traditional techniques, fs-laser WG writing in a glass chip offers two striking advantages: (i) it is a direct maskless fabrication technique, i.e., one can create in a single step optical WGs or more complicated photonic devices (splitters, interferometers, etc.) by moving the sample with respect to the laser focus; (ii) it is a three-dimensional technique, since it allows to define WGs at arbitrary depths inside the glass. Until recently this technique has been almost exclusively applied to the manufacturing of optical WGs for telecom photonic devices [Osellame, 2006] [Eaton, 2006]. However, it appears highly suited for the integration of optical WGs into LOCs. In fact, it allows to position optical WGs at arbitrary positions inside an already fabricated LOC without affecting the manufacturing procedure of the MF part of the device, thus greatly simplifying the production process and taking advantage of the already well developed technologies for MF chip fabrication. The optical WGs utilized during the course of this thesis were integrated with the MF channels in a standard CE LOC at the Politecnico di Milano, resulting in an integrated optofluidic chip aimed at fluorescent analysis of electrophoretically separated DNA molecules, as described in chapters 2-5 of this thesis.

\subsection{Outline of this thesis}

The broad theme of this thesis is the optofluidic integration in lab-on-a-chip devices to, in principle, enable various biochemical analyses to be performed in miniaturized microfabricated chips, thanks to the integrated optical sensing functionality. Having introduced the relevance of this field and its state of the art, the second chapter is then devoted to the description of the fabrication of the monolithic optofluidic chip. The third chapter reports on electrophoretic DNA sorting as performed in such a chip with high sensitivity and separation resolution. The fourth chapter is devoted to describe step by step the enhancement of the signal to noise ratio (SNR), and thereby the reduction of the limit of detection (LOD) in our setup, eventually enabling one to detect low concentrations of end-labeled DNA molecules during $\mathrm{CE}$ separation. In the fifth chapter, these individual results culminate in a qualitative improvement of the state of the art in fluorescent DNA analysis - thanks to our unique wavelength-multiplexing approach.

In chapter 2, the technologies for the cleanroom-based fabrication of the microfluidic chip are described. This development was largely the work of LioniX BV [Lionix, web] and has been commercialized as off-the-shelf 
microfluidic chips. Furthermore, the fabrication of optical waveguides for fluorescence excitation, integrated with the microfluidic analysis channels, is described. This work was performed with the fs laser post-processing approach by the group of Prof. G. Cerullo at the Politecnico di Milano [Martinez Vazquez, 2009]. Chapter 2 concludes with the description of the results of preliminary experiments to test the quality of the optical waveguides and the optofluidic integration in the context of DNA flow experiments with fluorescence monitoring. The results were promising and signaled toward flow experiments with a larger number of DNA analyte molecules at lower concentrations, i.e., approaching the real-life applications.

In chapter 3, the optimization of the various microfluidic parameters, e.g. electroosmotic flow suppression by means of channel-wall coating, DNA molecular sieving, etc., is described, which enabled us to reach the performance of the state of the art in electrophoretic DNA analysis. With this high performance, the optofluidic chip was in turn employed to carry out the electrophoretic sorting of 17 DNA molecules fluorescently labeled in situ with an intercalating dye. A high sizing accuracy, separation resolution, and sensitivity are achieved, paving the way for the higher-complexity analysis demonstrated in the chapter 5.

In chapter 4, the focus shifts to the enhancement of sensitivity, specifically to enable the detection of low concentrations (as encountered in real-life clinical diagnostic tests) of end-labeled DNA molecules. This is achieved, among others, by means of an all-numerical lock-in amplifier implementation. This enables a radical shift from the weakly bonding, transitorily and non-selectively attaching/detaching intercalating dyes to permanently, selectively, exclusively and covalently bonding end-labels.

In chapter 5, the optimized microfluidic protocols described in chapter 3 are combined with the ultrasensitive fluorescence detection from chapter 4. Furthermore, thanks to modulation-frequency encoding of the excitation laser beams and subsequent Fourier analytical decoding of the measured resulting fluorescence signals, multiplex genetic diagnostics is made possible. In the described example, the fluorescence signals from two independently end-labeled (color-coded) genetic probe sets, MCE-separated in a single run, under selective multi-wavelength laser excitation, were unambiguously unraveled from the cumulative signal measured by a colorblind photomultiplier tube. The final results described in chapter 5 represent a potential paradigm shift toward multi-color fluorescent DNA analysis, thanks to the novel method of modulation encoding/decoding, and therefore bear the potential to be an important step forward in MCE-based fluorescent DNA analysis. 


\section{Optofluidic integration in a lab on a chip}

\subsection{Introduction}

In the previous chapter we discussed the potential of integrated optofluidics as a method for addressing a part - namely the integration of optical waveguides (WGs) for fluorescence excitation in microfluidic (MF) channels - of the much more complex complete task of monolithic sensor integration in lab-on-a-chip (LOC) devices for biochemical analysis. This served as an introduction to the topics covered in this thesis focusing on integration of the excitation part. In this chapter, we will describe in detail how the optofluidic chip utilized in the experiments described in the remainder of this thesis is fabricated. This chapter is organized as follows: in section 2.2.1 we will describe the fabrication of the MF chip by standard, clean-room based batch fabrication technologies, followed in section 2.2 .2 by the femtosecond (fs-) laser writing of optical WGs as a post-processing on the MF chip. In section 2.3 we will describe the optical characterization of fs-laser written WGs in the context of fluorescence sensing applications within this thesis, continuing in section 2.4 with preliminary tests to demonstrate the optofluidic functionality of the chip in static and dynamic experiments - in ambiences that resemble a real biochemical-analysis and diagnostic environment - and the results thereof. The chapter will be concluded in section 2.5 with a summary of the achieved results.

\subsection{Integrated optofluidic sample fabrication}

In this section we discuss the fabrication steps involved in arriving at an optofluidic chip with integrated WGs for laser-excitation to induce fluorescence during biochemical analysis, starting from a mere fused silica wafer, upon undertaking two major processing steps, namely the fabrication of MF channels, followed by fs-laser inscription of monolithic optical WGs.

\subsubsection{MF chip fabrication}

The MF chips used within the context of this thesis were fabricated in fused silica glass substrates using conventional cleanroom-based technologies [Herold, 2009] derived directly from the semiconductor industry- addressing the issue of future mass production demands [Whitesides, 2006] - and made available by our industry partners within the EU project HIBISCUS [Hibiscus, web], namely LioniX BV [Lionix, web] and CapiliX BV [Capilix, web]. The reason behind choosing a somewhat more costly material, namely 
glass, in place of various other conventionally popular lab-on-a-chip materials such as the polymers poly-(dimethylsiloxane) (PDMS) [McDonald, 1999], poly-(methyl methacrylate) (PMMA) [Brown, 2006], etc., was the ease of transfer to high-volume production cycles, over-the-years tried-andtested biocompatibility, as well as the direct applicability of various biochemical / fluidic protocols developed traditionally for glass capillaries, i.e., assuming glass as the inert material that acts as the substrate for various biochemical processes.

The choice of fused silica over other alternatives such as borosilicate, pyrex, etc., has advantages such as transparency over the entire ultraviolet (UV) and visible region of the spectrum to enable a wide range of fluorescence sensing applications [Schott, web]. Photolithographic patterning followed by wet etching by $33 \% \mathrm{HF}$ - as standard batch processing on a set of fused silica wafers (Schott Lithosil) - is employed to create a network of MF channels connected to the outside world via MF reservoirs. Two different designs were employed during this work (as will be described in greater detail in chapter 3), which also included different cross-section dimensions of the MF channel, namely 50x12 $\mathrm{m}^{2}$ (design 1, Fig. 2.1(a)) and $110 \times 50 \mu \mathrm{m}^{2}$ (design 2, Fig. 2.1(b-c)) (breadth $\mathrm{x}$ height). In addition, a small variation in design 2 consisted of a so-called "double-T" (Fig. 2.1(c)) type MF crossing junction in order to increase the volume of the injected MF plug without affecting the duration of injection. In the following step an unpatterned fused silica wafer was fusion bonded at $1100^{\circ} \mathrm{C}$ on top of the patterned fused silica wafer, as a cover glass substrate, in order to direct the flow of the contents of the MF channels, and in order to isolate the MF channels from dust, dirt and other sources of particulate contamination from the ambient environment to prevent clogging of the MF channels. Individual chips of dimensions $64 \times 5.5 \times 1 \mathrm{~mm}^{3}$ were diced out, and their side-facets were polished to facilitate low-loss optical in-out coupling with fibers. Figs. 2.2-2.4 show these chips in photographs. 
(a)

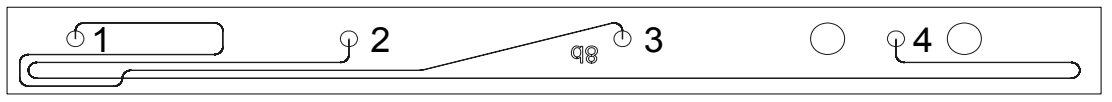

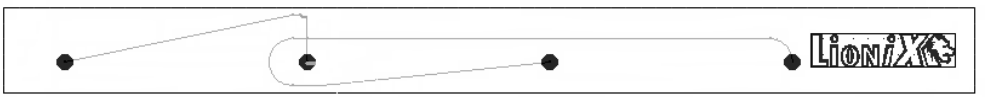

(b)
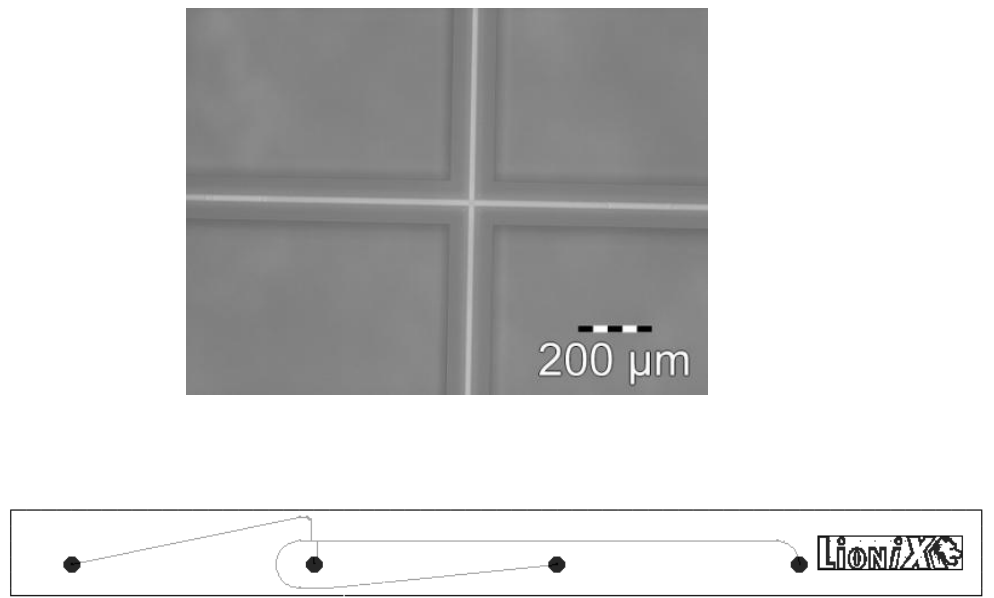

(c)

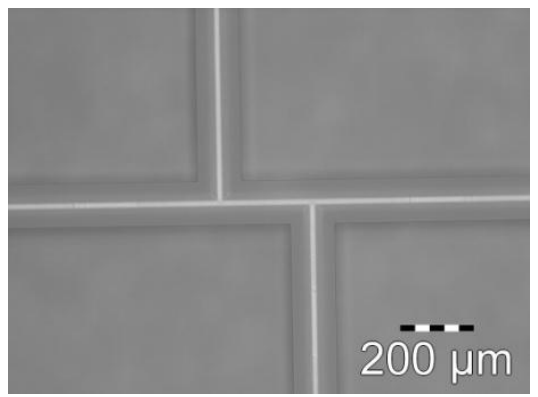

Fig. 2.1 MF chip designs having MF channel cross-section dimensions of (a) $50 \times 12 \mu^{2}$ and (b) $110 \times 50 \mu^{2}$ (conventional MF crossing junction) as well as (c) $110 \times 50 \mu^{2}$ (double-T MF crossing junction) (courtesy of LioniX BV [LioniX, web]) 


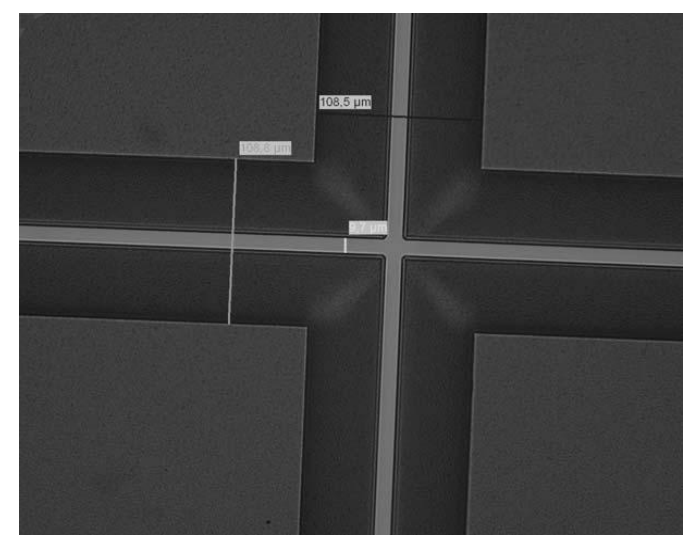

Fig. 2.2 SEM image showing the MF crossing junction in design 2 chip; note the well-defined, low surface-roughness under-etched channel (courtesy of Lionix BV [LioniX, web])
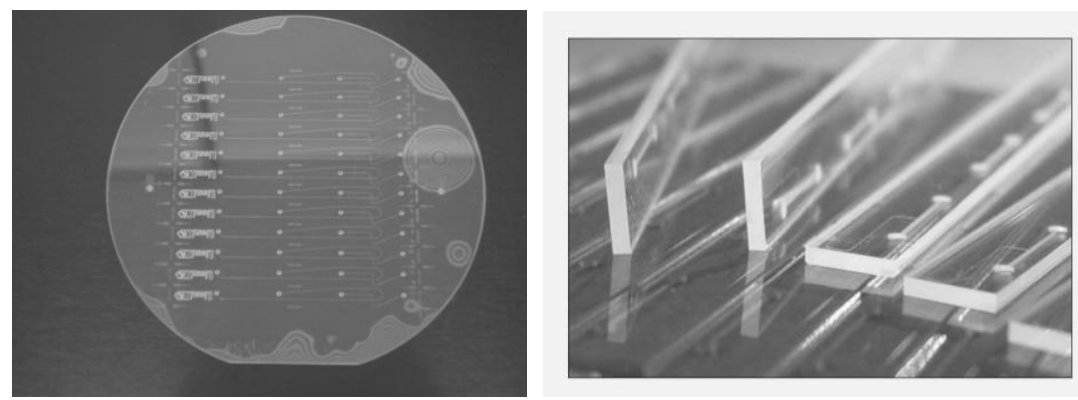

Fig. 2.3 MF channel networks on a fused silica wafer, after fusion bonding and before dicing (left); and individual chips diced out from the fusion bonded wafers (right) (courtesy of LioniX BV [LioniX, web])

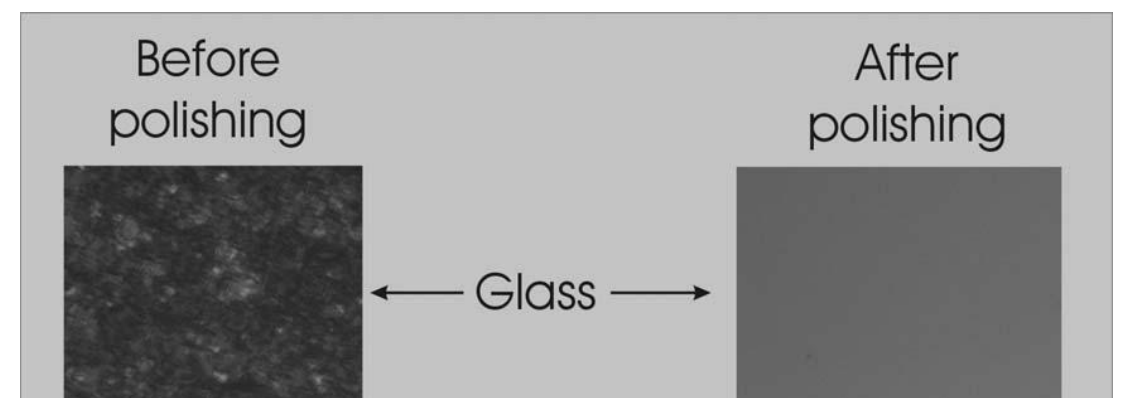

Fig.2.4 Comparison between images obtained by an optical microscope of the end-facets of the diced MF chips before and after polishing; the surface after polishing is optically smooth (mean surface roughness $<\lambda / 8$ ) (courtesy of LioniX $B V$ [LioniX, web]) 


\subsubsection{Optical post-processing by fs-laser WG writing}

The previous chapter described a number of different approaches to achieve optofluidic integration [Lien, 2004] [Leeds, 2004] [Lien, 2005] [Cleary, 2005] [Psaltis, 2006] in a LOC device aimed at biochemical analysis [Lambeck, 2006] [Verpoorte, 2003] [Yin, 2006] [Applegate, 2007] [Yin, 2007] [Mazurczyk, 2006]. The advantages of monolithic post-processing for exploiting the existing, mature MF fabrication infrastructure were described. Such a post-processing approach would be more viable over hybrid integration, which involves the invocation of other materials to introduce certain functionalities in addition to the MF functionalities. This is also one of the justifications for making use of fs-laser enabled optofluidic integration [Osellame, 2007] over a number of other approaches addressed in the literature utilizing different material platforms, e.g. silica on silicon [Ruano, 2000] [Friis, 2001] [Dumais, 2005], polymers [Mogensen, 2003] [Lee, 2003] [Wang, 2006], liquid core [Bliss, 2007] [Olivares, 2002] [Dumais, 2006], SiON [Mogensen, 2001], etc. This resulted in the fs-laser micromachining [Liu, 1997] [Gattass, 2008] approach and the resulting MF chips with integrated WGs being used during the course of this work. In this section we describe fs-laser WG writing [Davis, 1996] [Miura, 1997] in fused silica [Bellouard, 2004] LOCs. This WG fabrication technology was implemented within the group of Prof. G. Cerullo, at the Politecnico di Milano (partners within the EU-project HIBISCUS) and processed chips were provided by them for this thesis work.

Figure 2.5 shows the setup used for fs-laser writing of optical WGs in glass [Martinez Vazquez, 2009]. A regeneratively amplified Ti:sapphire laser (model CPA-1, Clark Instrumentation) producing $150 \mathrm{fs}, 500 \mu \mathrm{J}$ pulses at 1 $\mathrm{kHz}$ repetition rate and $800 \mathrm{~nm}$ wavelength is used as the fs-laser source [Osellame, 2004] [Osellame, 2007]. The original laser pulse energy, attenuated to $2-5 \mu \mathrm{J}$, controlled by a variable attenuator, is used for WG writing. The pulses are focused by a low numerical aperture microscope objective (NA $\sim 0.3$ ) at a depth variable from 0.2 to $1 \mathrm{~mm}$ inside the glass. The samples are moved in three dimensions by a precision translation stage (Physik Instrumente model M-155.11). The ability to induce such arbitrary 3D movement of the sample with respect to the writing laser beam enables the unique 3D WG fabrication [Nolte, 2003] [Gleezer, 1996] [Marcinkevicius, 2001] [Bellouard, 2004] [Martinez Vazquez, 2009] [Crespi, 2010] [Zhang, 2008]), at speeds ranging from 10 to $100 \mu \mathrm{m} / \mathrm{s}$, perpendicularly to the beam propagation direction (transverse writing geometry). 

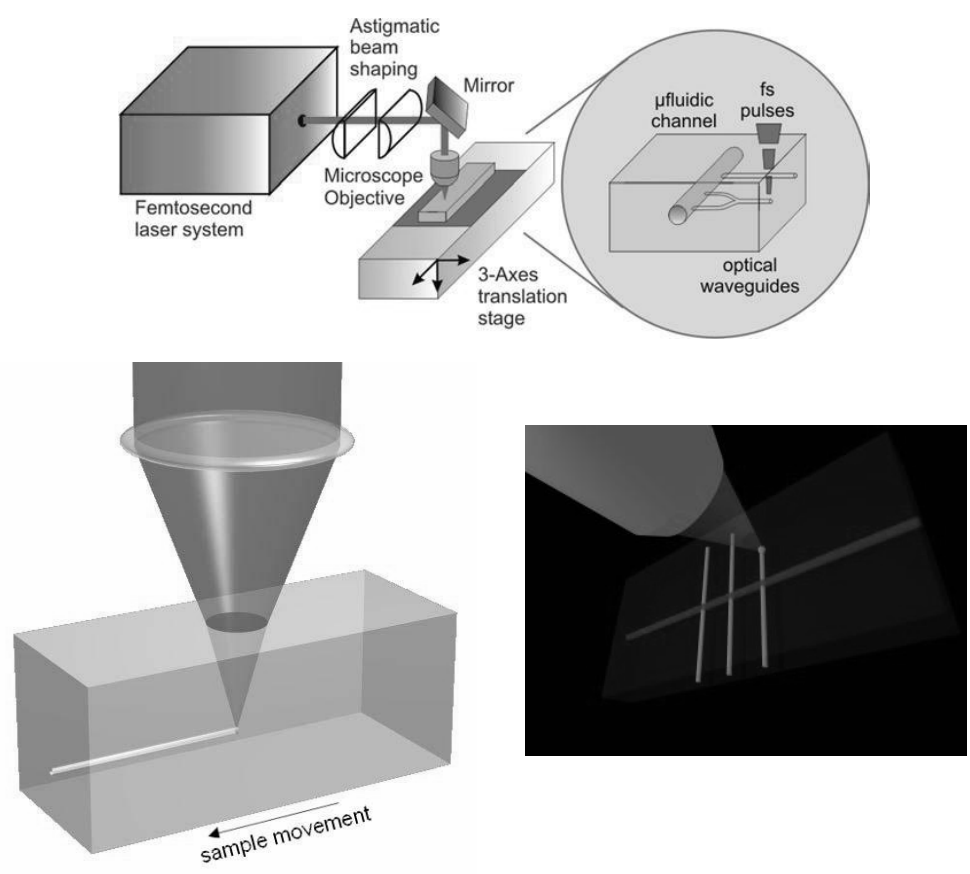

Fig. 2.5 (top) Experimental setup for fs laser WG writing in the bulk of a fused silica substrate, (bottom left) WG writing in a fluidically unpatterned substrate, (bottom right) $W G$ writing in a patterned substrate to cross a MF channel perpendicularly in plane [Martinez Vazquez, 2009]

This geometry ensures fabrication flexibility, but the use of a single focusing objective leads to WGs with a strongly asymmetric cross section. This is overcome by introducing a focusing geometry in which the fs-laser writing beam is astigmatically shaped [Cerullo, 2002] by changing both the spot sizes in the tangential and sagittal planes as well as the relative positions of the beam waists within the focal volume. This shaping allows one to modify the interaction volume in such a way that the WG cross section can be made circular or elliptical and with varying size [Osellame, 2003]. Such astigmatic beam shaping can be easily obtained by a cylindrical telescope; fine control of the distance between the cylindrical lenses is used to tune the offset between the two positions of the beam waists corresponding to the two transverse directions and thus the WG cross section. The WG fabrication process was optimized on plain fused silica substrates similar to those used for the mass production of the LOC devices.

The next step in the optofluidic sensor integration in the LOC was the integration of such optical WGs in real LOC devices capable of biochemical analyses, to enable selective excitation and probing of the content of the MF channels. A commercial electrophoretic microchip (design 1) (D8-LIF from 
CapiliX BV) was used for this purpose. The chip layout is shown in Fig. 2.1(a). It consists of two MF channels mutually crossing each other in plane, but folded in a complex way in order to reduce the chip's footprint, that are responsible for the sample injection (MF channel going from reservoir 1 to 3 ) and for the electrophoretic separation (MF channel going from reservoir 2 to 4). The separated MF plugs at the end of channel 2-4 would conventionally be detected by laser-induced fluorescence using a confocal microscope [Jiang, 2000] [Leica, web] [Lundqvist, 2003].

A series of WGs perpendicular to the CE separation channel were fabricated by means of fs-laser material processing, toward the end of the $\mathrm{CE}$ separation channel. We have chosen for a positioning of the WGs at different points in order to have some freedom in selecting the most suitable position for fluorescence excitation/detection. The MF channels lie $500 \mu \mathrm{m}$ below the chip surface and are rather small, with a rectangular cross-section measuring $50 \mu \mathrm{m}$ in width and $12 \mu \mathrm{m}$ in height for the original chip design. The positioning of the optical WG with respect to the MF channel is thus quite challenging, in particular in the depth direction where the MF channel dimension is relatively small.

Figure 2.6(b) shows the differential interference contrast microscope images of the optical WGs' end-view together with the MF channel. From left to right, the position control can be appreciated, with one WG slightly above the MF channel, one slightly below (both can be used for evanescent field sensing applications), and three perfectly centered (in the third image the MF channel is out of focus to allow visualization of the centered optical WGs) WGs for the fluorescence sensing applications presented in this thesis.

To prevent damaging of the MF channel walls when the laser crosses them, the writing beam was interrupted a few $\mu \mathrm{m}$ before the channel and restored just after it, by synchronizing a beam shutter with the driver of the translation stage. Stopping the WGs a few $\mu \mathrm{m}$ before the channel does not cause any loss of spatial resolution during fluorescence excitation, since, due to the low numerical aperture of the WGs, the Rayleigh range of the light coupled out of them exceeds $50 \mu \mathrm{m}$ and thus the excitation remains quite confined when crossing the MF channel with negligible divergence effects. It is also important to note that, despite the fact that the WGs are written very close to the bonding surface between the two glass slabs of the MF chip, no detectable damage was induced. This indicates that the bonded glass slabs behave as a bulk piece of fused silica for all practical WG-writing purposes and the fs-laser writing process is compatible with the bonding procedure. This result is critical for the optical integration in any existing MF chip, consisting of a substrate with MF channels bonded to a cover glass for closing the channels. These results demonstrate that the direct inscription technique allows the integration of buried optical WGs on a functional MF 
chip. This post-processing capability provides strong design flexibility for the photonic devices to be integrated.

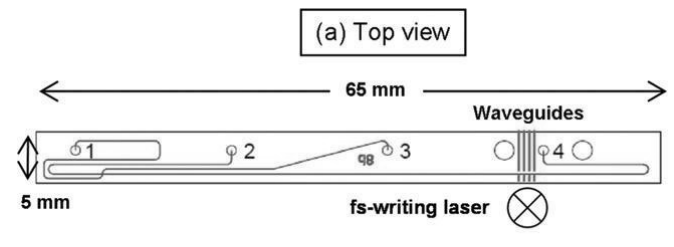

(b) Side view

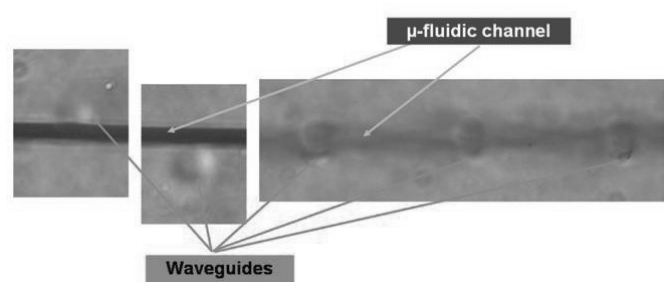

Fig. 2.6 Images depicting $f_{s-l a s e r} W G$ writing in a MF chip (design 1) in (a) layout / top-view, and (b) side-view [Martinez Vazquez, 2009]

A typical translation speed of $20 \mu \mathrm{m} / \mathrm{s}$ is used for $\mathrm{WG}$ writing. This rather low fabrication speed may be considered the main limitation of this technology. Higher speeds have been demonstrated with higher repetition rate lasers [Eaton, 2006] [Osellame, 2005]; however, even with the above value, a 4-mm-long WG is manufactured in each chip in about 3 minutes. The modified volume presents a circular cross section with a diameter of approximately $12 \mu \mathrm{m}$ (in MF chip design 1), matching the MF channel depth, and an elliptical cross section (in MF chip design 2) with a minor diameter of $12 \mu \mathrm{m}$ (to ensure a high spatial resolution) in the horizontal direction and a major diameter of $50 \mu \mathrm{m}$ in the vertical direction (to excite fluorescence along the entire MF channel depth, thereby maximizing the generated signal to be detected). With the fabrication parameters described here, the material modification is limited to the focal volume of the writing beam. This is important for this specific application since the relative position of optical WG and MF channel is critical, calling for a fine control of position and shape of the modified region.

\subsection{Characterization of the integrated WGs}

In this section we discuss the optical characterization performed at the Integrated Optical Microsystems group, University of Twente, of the fs-laser written WGs integrated in the MF chip for fluorescence sensing applications. 
Parameters such as single- / multi-mode behavior, refractive-index profile of the WG cross section, and their propagation losses will be discussed and compared with existing methods. The outcome of these characterizations will serve as important pointers to the applicability of this WG fabrication technique in optical sensing.

\subsubsection{Near-field imaging}

The optical WGs were excited by in-coupling light at $633 \mathrm{~nm}$ through a 9 $\mu \mathrm{m}$ fiber. The near field mode profiles at the output of these WGs were projected with a $25 \mathrm{x}$ microscope objective onto a grayscale CCD camera (10-bit pixel resolution). It was observed that in the chip design 1 (MF channel cross-section $=50 \mu \mathrm{m} \times 12 \mu \mathrm{m}$ ), the WGs were single-mode (for given polarization) as the outgoing field was observed to be independent of the exact in-coupling conditions- see Fig. 2.7., The modal field has a circular cross-section, owing to the astigmatic beam shaping described in section 2.2.2. Single-mode operation is however not necessary for fluorescence excitation and the subsequent devices indeed implemented multi-mode WGs in order to excite fluorescence in the contents of a much larger effective volume of the MF channel.
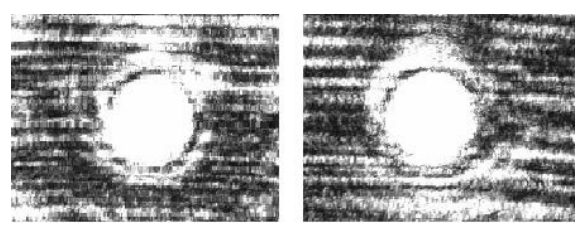

Fig. 2.7 Near-field mode profiles of two WGs inscribed in the MF chip (design 1) with a linear CCD camera through a $25 x$ microscope objective

It was observed, as expected, that most of the WGs in the chip design 2 were in fact multi-mode, and displayed a variety of shapes and sizes, dependent on the in-coupling conditions, as evident from the sets of CCD camera images in Fig. 2.8. It was also observed that the near-field profile that could be excited was dependent on the position of the in-coupling fiber in $\mathrm{x}$ y plane w. r. t. the WG input end face. 

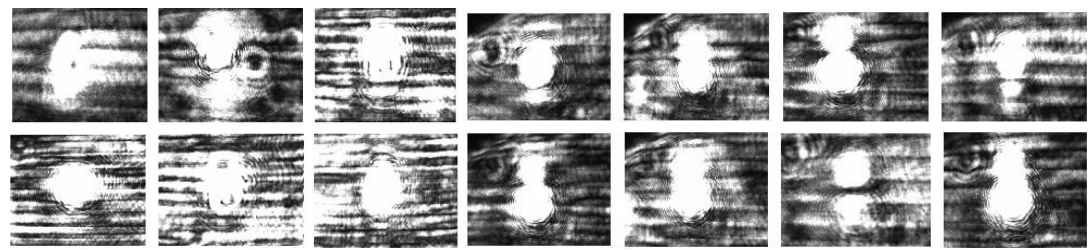

Fig. 2.8 Near-field mode profiles of WGs inscribed in the MF chip (design 2) with a linear CCD camera through a $25 x$ microscope objective

In Figs. 2.7 and 2.8, one can notice the presence of interference fringes in the horizontal direction, resulting from the back-reflections from the top and bottom surfaces of the chip. This is an important reason why these near-field images cannot be used to perform further calculations to determine, e.g., the refractive index contrasts in the WG cross section. In the next section we will describe a technique to eliminate such interference and obtain the refractive index contrasts of these WGs by means of a simple back-calculation algorithm.

\subsubsection{Refractive index profile}

The technique used to evaluate the $2 \mathrm{D}$ refractive index profile in the transverse plane makes use of an algorithm to back-calculate the index distribution as a function of the near-field mode-profile at the output end face of the WG in question [Bibra, 1997]. While the CCD camera images in the previous section provided valuable information concerning the single- / multi-mode behavior of the WGs, the low resolution and the presence of interference fringes lead to the need for another technique to capture the near-field profiles for refractive-index reconstruction. We made use of a scanning fiber tip in collaboration with the Optical Sciences group, University of Twente. In this technique, the WG cross-section at the output end face is directly aligned with a 110-nm near-field scanning (SNOM) fiber tip [Veerman, 1998]. The tip was mounted on automated movement stages (Newport) with a movement resolution of $20 \mathrm{~nm}$. A labview program controlled this setup to automatically align the WG with the fiber tip, by means of a simultaneous atomic force microscopy (AFM) measurement to provide feedback concerning the distance between the WG cross-section and the outcoupling fiber tip, thereby maintaining the separation at a constant 10 $\mathrm{nm}$. On optimal alignment, the out-coupling fiber was used to scan the nearfield mode profile in the $x-y$ plane with a spatial resolution down to $20 \mathrm{~nm}$. The resulting field profile is measured by an optical power meter which feeds the data back to the measurement software, as shown in Fig. 2.9. 


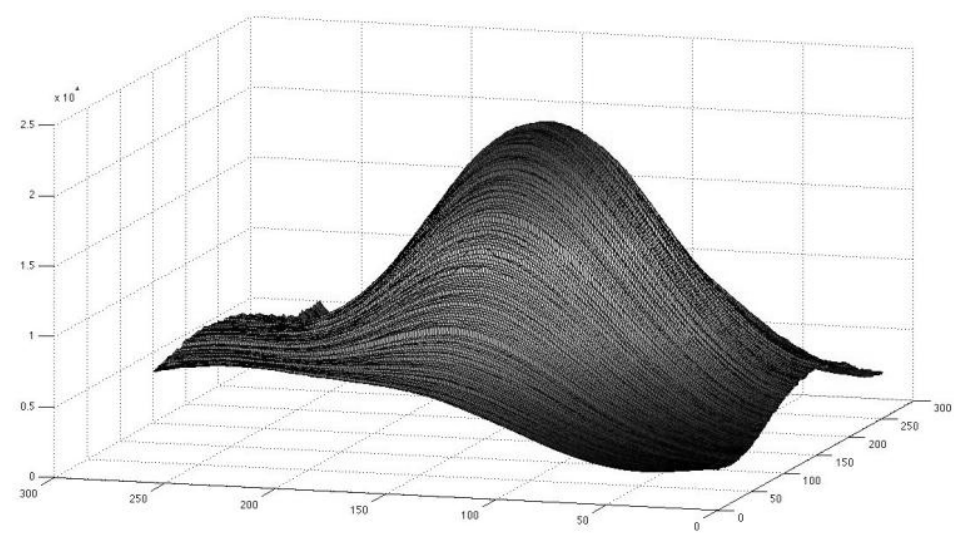

Fig. 2.9 Near field mode profile of the fundamental mode in a fs-laser written $W G$ captured by the automated fiber tip scanning technique

The following back-calculation paradigm can be used to evaluate the index profile based on the captured/scanned near-field mode profile, provided that assumptions such as weak guidance, graded index variation, and a low index contrast hold true, which they do in this case. Assuming that the WG is oriented along the $z$-axis, we have the scalar eigen-value equation for the modal field,

$$
\nabla_{T}^{2} \Psi(x, y)+\left(k_{0}^{2} n^{2}(x, y)-\beta^{2}\right) \Psi(x, y)=0,
$$

where $\Psi(x, y)$ is the dominant component of the modal field, $\beta$ is the propagation constant, and $n(x, y)$ is the refractive index profile to be determined. Rearranging the above equation, we receive

$$
n^{2}(x, y)=\left(\frac{\beta^{2}}{k_{0}^{2}}\right)-\frac{\nabla_{T}^{2} \Psi(x, y)}{k_{0}^{2} \Psi(x, y)} .
$$

Substituting the modal field intensity $I(x, y)=\Psi^{2}(x, y)$, and $n(\mathrm{x}, \mathrm{y})=n_{B}+$ $\Delta n(\mathrm{x}, \mathrm{y})$ where $n_{B}$ is the refractive index of the bulk material, and assuming that $(\Delta n)^{2}<<2 n_{B}$, we obtain

$$
\Delta n(x, y)=\frac{\beta^{2}}{2 n_{B} k_{0}^{2}}-\frac{n_{B}}{2}-\frac{\nabla_{T}^{2} \sqrt{I(x, y)}}{2 n_{B} k_{0}^{2} \sqrt{I(x, y)}} .
$$


Software was developed in-house to implement the evaluation of refractive index profile in this manner, and Fig. 2.10 describes the result.

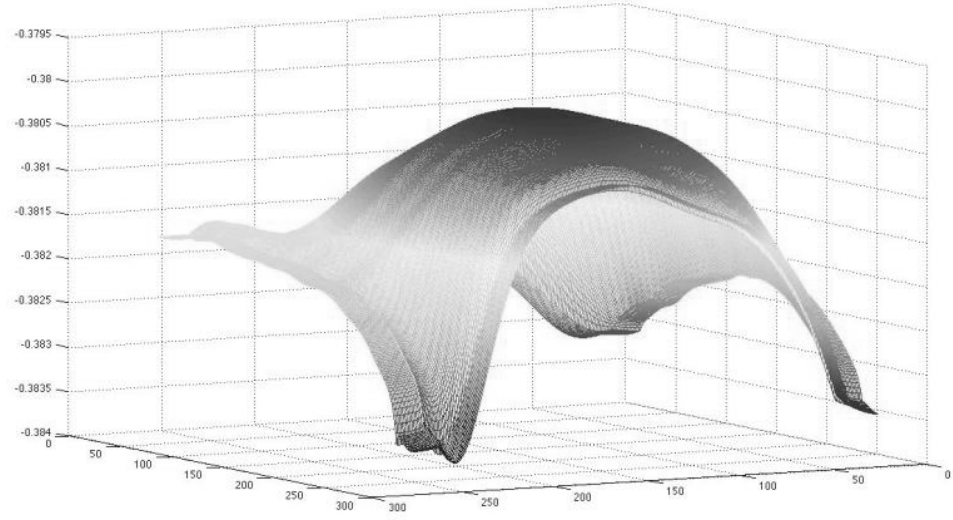

Fig. 2.10 WG cross section refractive index profile based on its near field mode profile

The refractive index profile as well as the index contrasts evaluated by this method $(\sim 0.0009)$ at $633 \mathrm{~nm}$ matches quite well with the results of the index profile measurements carried out by using a commercial index profilometer (Rinck Elektronik GmbH, Jena, Germany) as shown in Fig. 2.11. This value is in turn in good agreement with the results of a calculation using a numerical mode solver software (BeamPROP 4.0, RSoft) to reproduce the measured WG mode. This low refractive index change implies a limited numerical aperture of the WG (NA 0.05). As will be discussed in the following section (2.4) on the optofluidic characterization of the chip, this is beneficial for uniform excitation through the MF channel. Nevertheless, the refractive index change may be increased with different irradiation parameters such as higher (up to $1 \mathrm{MHz}$ ) repetition rates, to reach values up to $\sim 1 \times 10^{-2}$, in order to enable the writing of sharper bends, e.g. to fabricate optical power splitters. 


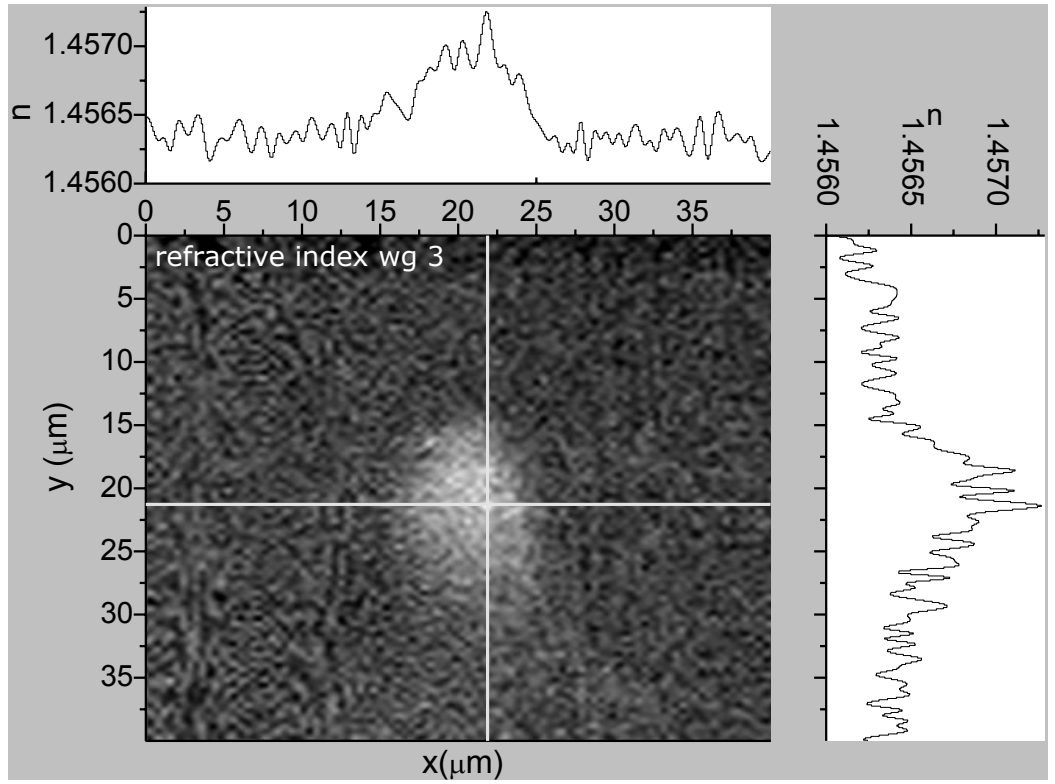

Fig. 2.11 Refractive index profile obtained by a commercial index profilometer for a fs-laser written WG (courtesy of Politecnico di Milano)

\subsubsection{Propagation loss}

Propagation loss at $543 \mathrm{~nm}$ was measured using the cut-back technique [Reed, 1992] at the Politecnico di Milano, and was found to be $0.9 \mathrm{~dB} / \mathrm{cm}$, as shown in Fig. 2.12.

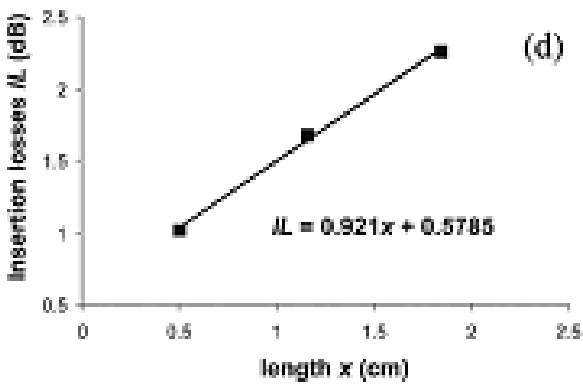

Fig. 2.12 Propagation loss measurement at $543 \mathrm{~nm}$ performed by cut-back method on a fs-laser written WG; (the plotted straight line does not pass through the origin owing to the fiber-chip in-coupling losses) [Martinez Vazquez, 2009]

This value was confirmed by means of the "streak of luminescence" technique [Okamura, 1983], where a top-view CCD-camera image of the WG (Fig. 2.13 top-left) was obtained by capturing the luminescence emitted 
along the WG length, resulting from the 633-nm excitation of the colorcenters (Fig. 2.13 top-right) created during the fs-laser writing. From the linear decrease in the logarithm of the luminescence intensity, we derived the propagation loss of the WG, as shown in Fig. 2.13 (bottom). This value (0.9 $\mathrm{dB} / \mathrm{cm})$ is very promising as compared to those obtained at similar wavelengths on other kinds of WGs integrated on LOCs and fabricated with SiON ([Mogensen, 2001] $1 \mathrm{~dB} / \mathrm{cm}$ ), SU-8 polymer ([Mogensen, 2003] 2.5 $\mathrm{dB} / \mathrm{cm}$ ), and liquid core ([Bliss, 2007] $1.8 \mathrm{~dB} / \mathrm{cm}$ ) technologies. The performance of the WGs obtained in plain fused silica substrates thus appeared fully adequate for implementation in real biochemical LOCs in terms of mode profiles and propagation losses.
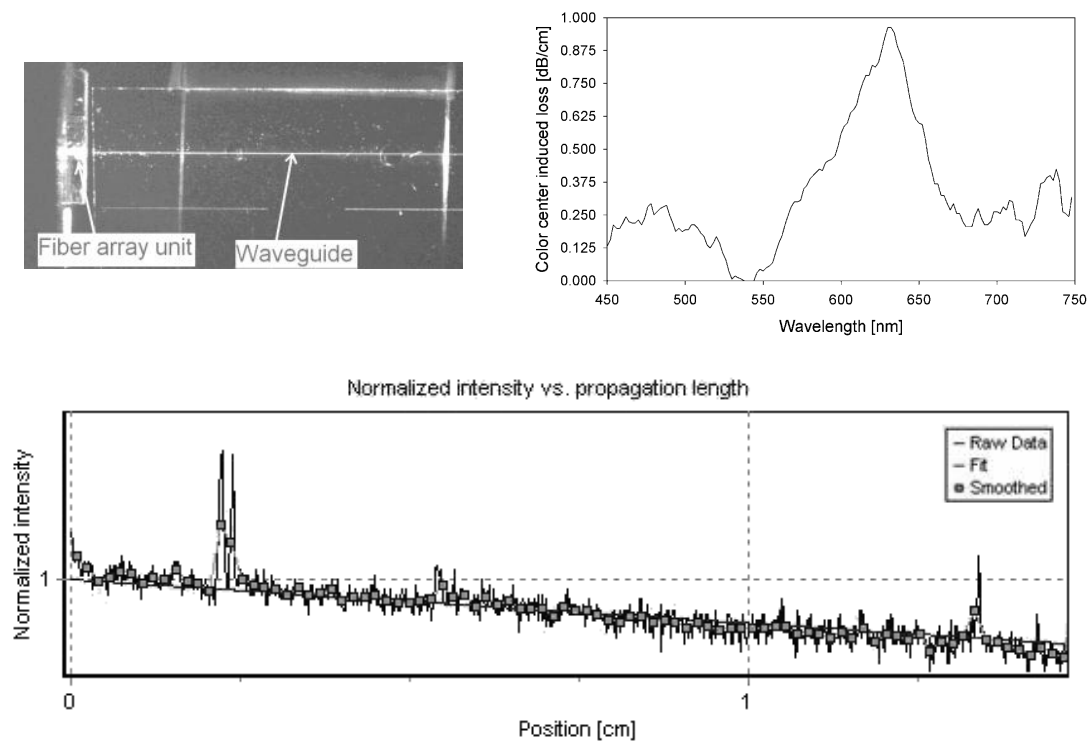

Fig. 2.13 Streak of luminescence technique for the evaluation of propagation losses in a fs-laser written WG. (a) Top-view of a WG carrying light at $633 \mathrm{~nm}$ and thereby illuminated owing to color-centers created during laser writing; (b) luminescence spectrum of the light captured from the top of the $W G$; (c) linear decrease as a function of $W G$ length in the luminescence intensity emitted by the $W G$ and captured by the CCD camera in top-view

\subsection{Optofluidic characterization of the lab on a chip}

In this section, we will discuss the various preliminary tests performed in order to evaluate the quality and functionality of the optofluidic integration. Specifically, these included a static test wherein a MF channel was filled with a strongly emitting fluorescent dye, and selectively, locally excited by the integrated WG intersecting the MF channel in plane [Martinez Vazquez, 
2009]. While the performance test during a dynamic flow experiment included capillary electrophoresis separation of two strongly emitting fluorescent dyes in the MF channel - their fluorescence being selectively, locally excited by the integrated WG.

\subsubsection{Sensing of static events}

In order to demonstrate the ability of the fs laser written optical WGs to excite fluorescence in the contents of the MF channel, the latter was filled with a solution of Fluorescein dissolved in MES/His (40 mM / $20 \mathrm{mM})$ buffer ( $\mathrm{pH}$ 6.2), used as model fluorescent dye. The $488 \mathrm{~nm}$ line from a continuous wave Argon ion laser beam (Spectra-Physics) was coupled into the integrated WG via an optical fiber array unit optimally aligned and glued to the end-facet of the chip.

Fig. 2.15(right) shows a microscope image of the green-yellow fluorescence for Fluorescein dye. The spatial resolution of the fluorescence excitation is $12 \mu \mathrm{m}$, as defined by the WG diameter. Only one stripe of light with a width of $12 \mu \mathrm{m}$ is observed over the entire width of the MF channel, indicating that there is low divergence of light as it exits out of the WG as it intersects the MF channel. This will become especially important for a high spatial resolution electrophoretic separation once the MF plug widths would become comparable to the WG dimensions, as will be discussed in chapter 3 . The results depicted in Fig. 2.15(right) thereby demonstrate the ability of the integrated optical WG to excite with high spatial selectivity the biochemical contents of the MF channel. The experimental setup to ensure a high sensitivity and a strong reduction of background noise as used for the laserinduced fluorescence sensing experiment shown in Fig. 2.15 is shown in Fig. 2.14. This setup is also used for monitoring the dynamic flow experiments described in section 2.4.2.

The CE microchip is inserted in a commercial MF cartridge providing electrical and fluidic connections to the on-chip MF reservoirs (MCC-1 of the Capella platform, CapiliX BV). The integrated WGs are addressed by means of fiber array units aligned and glued to the chip end-facets. The cartridge is placed into the MF docking station which in turn is placed on the sample stage mounted on an inverted microscope. The emitted fluorescence signal passes through an emission filter (XF57 from Omega Optical, Inc.) to a color CCD camera for visualization of on-chip events if desired, and to a photomultiplier tube (PMT) for sensitive measurement of the fluorescence signals. 

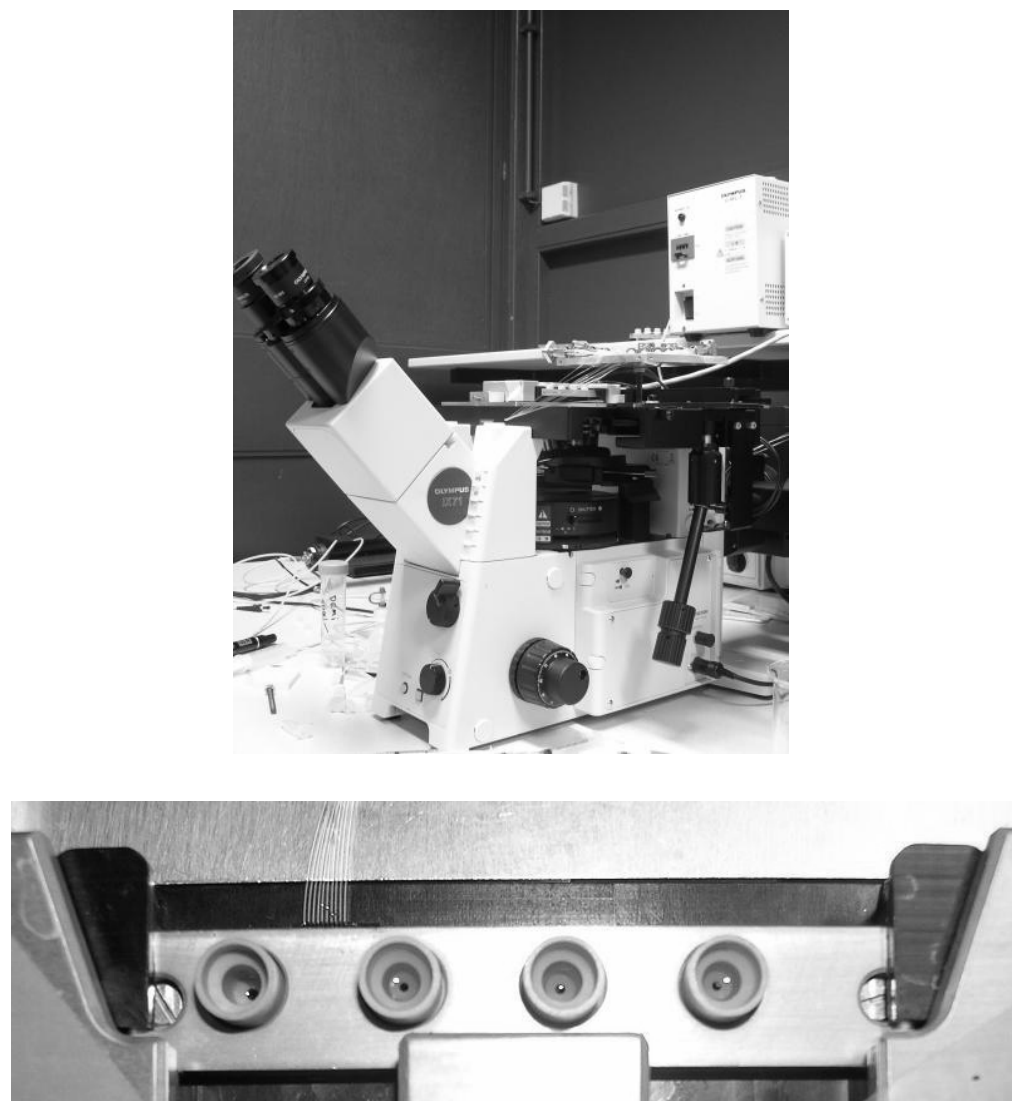

Fig. 2.14 Laboratory-based experimental setup for laser-induced fluorescence measurements
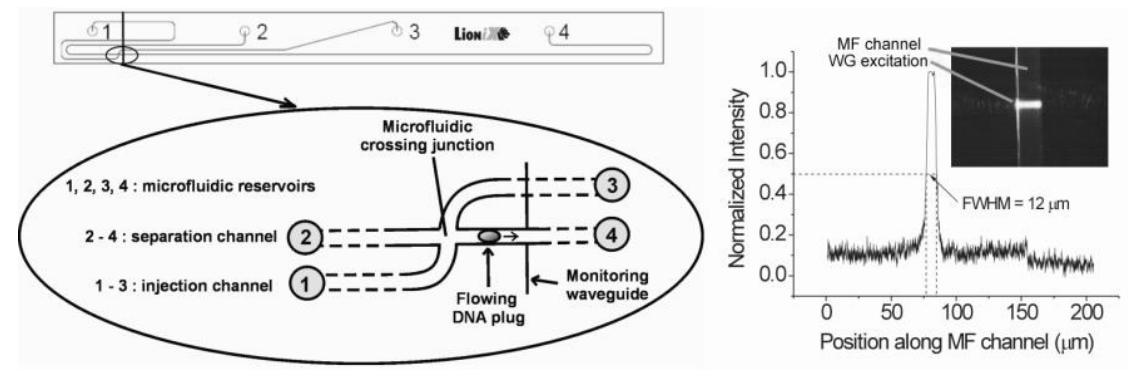

Fig. 2.15 (left) Schematic of the MF chip (design 1) with a fs laser written WG intersecting a MF channel right after the MF crossing junction to enable detection of small-sized molecules during electrophoretic separation (right) excitation of Fluorescein dye filling the MF channel (inset), and the emitted fluorescence intensity as a function of the position along the MF channel (indicating the $12 \mu \mathrm{m}$ spatial resolution) 


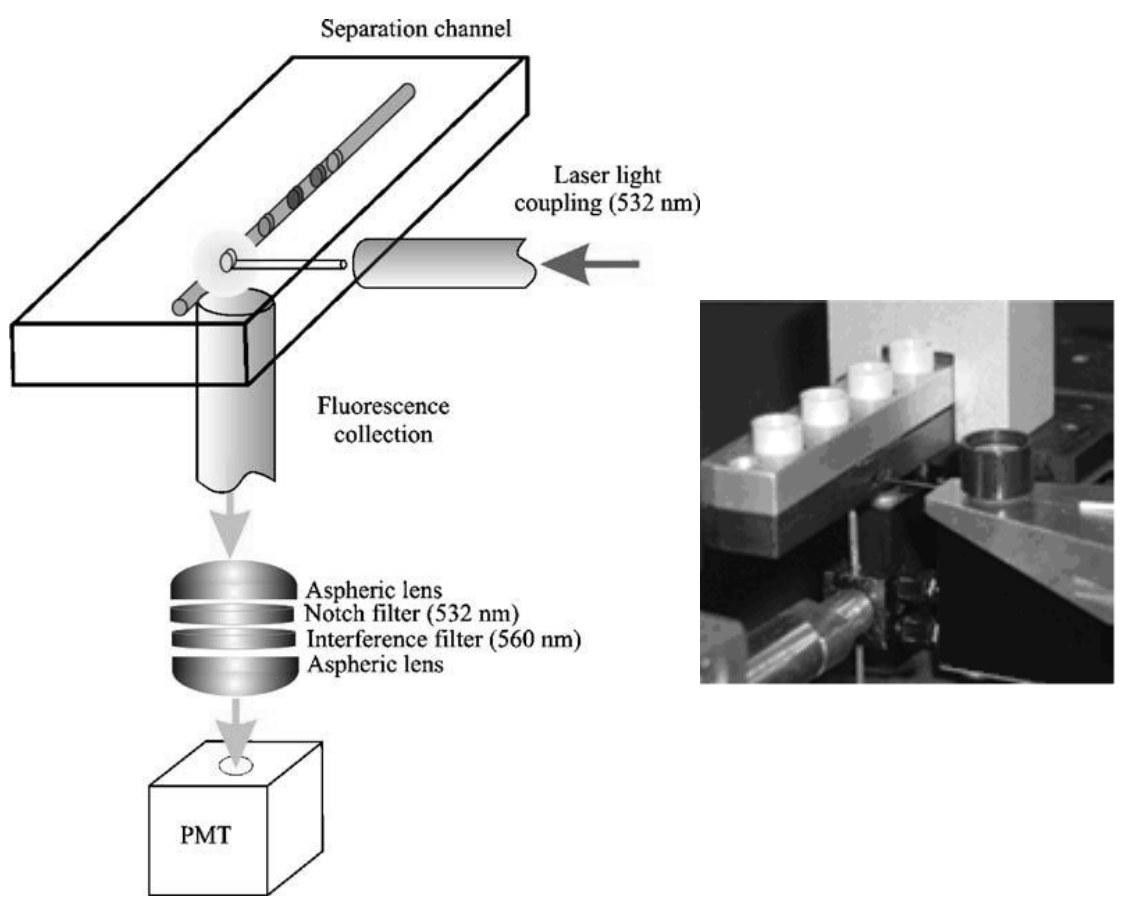

Fig. 2.16 (left) Schematic, and (right) picture depicting the compact fluorescence detection setup, based on a fluorescence-collection fiber [Martinez Vazquez, 2009]

In order to introduce further compactness and portability, modifications were applied to this setup as shown in Fig. 2.16, as a possible alternative in the future for field applications of this technology. These experiments were performed at / in collaboration with the Politecnico di Milano. In this approach, direct collection of fluorescence with a high numerical aperture optical fiber was implemented [Martinez Vazquez, 2009]. The fluorescence was then collected by an optical fiber pigtailed to the chip in correspondence to the excited portion of the MF channel, in 90-degree geometry with respect to the exciting WG, thus achieving a strong suppression of the excitation (background) signal. Both the numerical aperture and the diameter of the optical fiber would be selected in order to maximize the collected fluorescence and limit the effect of stray light. The light collection efficiency, defined as the fraction of isotropic fluorescence collected by the optical system, is given by $\mathrm{LCE}=\Omega / 4 \pi=1 / 2\left(1-\cos \theta_{\max }\right)$, where $\Omega$ is the solid angle subtended by the collection optics, corresponding to a half cone with angle $\theta_{\max }$. This angle can be related to the numerical aperture of the optical fiber by NA $=n_{0} \sin \theta_{\max }$, where $n_{0}$ is the refractive index of the medium from which the fluorescence is impending. One can then write [Martinez Vazquez, 2009] 


$$
\mathrm{LCE}=\frac{1}{2}\left(1-\sqrt{1-\left(\frac{N A}{n_{0}}\right)^{2}}\right),
$$

for NA $=0.5$ one achieves a LCE of $3 \%$, which is comparable to that of a confocal microscope objective - the competing bulky technique - with the advantage of potential further integration and system portability given by the fiber-based collection system. An additional constraint to be satisfied is that the fiber cross-section is large enough to intercept all the rays contanined within $q_{\max }$. Assuming the fluorescence as a point source located at a distance $d$ below the chip surface, and calling $a$ the fiber radius, one obtains

$$
a \geq d \tan \theta_{\max }=\frac{d \frac{N A}{n_{0}}}{\sqrt{1-\left(\frac{N A}{n_{0}}\right)^{2}}} .
$$

For the numerical values relevant to our case $\left(d=500 \mu \mathrm{m}, n_{0}=1.45\right)$ one obtains $a \geq 180 \mu \mathrm{m}$. The specific setup used a collection fiber with NA = 0.48 and a core radius of $300 \mu \mathrm{m}$ (model HWF-H-600T, Ceram Optec), which satisfies both requirements, has a fused silica core (to minimize autofluorescence) and a polymer cladding to provide high refractive index contrast. A possible alternative to collect the fluorescence signal would have been to use another fs laser written WG, albeit integrated orthogonally to the plane of the MF channels. The use of an optical fiber to collect the fluorescence, rather than a second inscribed WG, could be justified because of the following reasons: (i) in the geometry under consideration, the path from the excited volume to the microchip surface is only $500 \mu \mathrm{m}$, therefore a large core area fiber can easily intercept a wide solid angle of the emitted fluorescence; (ii) it is very difficult with any fabrication technology to integrate a WG with NA comparable to the fiber we used, thus, notwithstanding the closer collection allowed by a WG, a larger amount of fluorescence would have been lost; (iii) a high numerical fiber would however have been necessary to deliver the light collected by the WG to the detector. The core diameter of the collecting fiber being $600 \mu \mathrm{m}$, it greatly relaxes the alignment with the MF channel which can be performed by naked eye 
In order to assess the sensitivity of such a system, a measurement of the system's limit of detection was performed. To this purpose the chip was filled with progressively higher concentrations of Rhodamine 6G. Figure 2.17 shows the fluorescence as a function of concentration for an average power of $100 \mathrm{~mW}$ coupled into the WG. The curve displays an excellent linearity and shows the capability of this system to detect very low fluorophore concentrations, down to the $40 \mathrm{pM}$ level which corresponds to the presence of 150 individual fluorescence-emitting molecules in the excitation/detection volume.

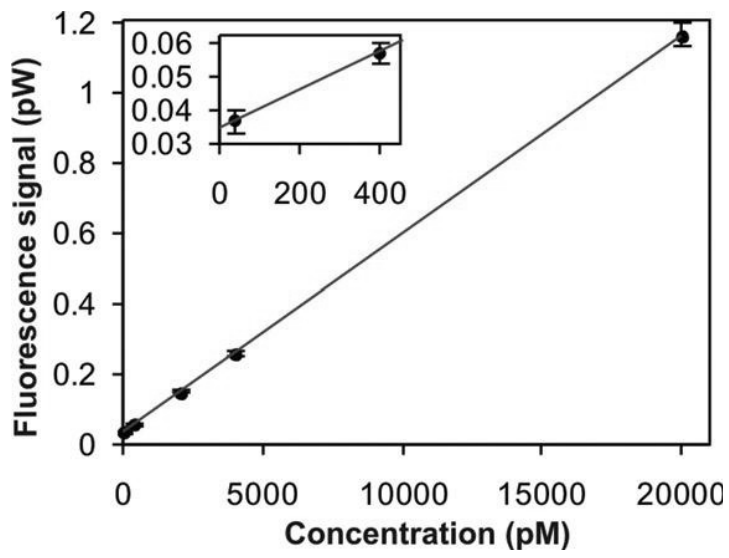

Fig. 2.17 Fluorescence intensity as a function of fluorophore concentration, depicting the limit of detection of the setup [Martinez Vazquez, 2009]

This limit of detection compares well with values reported in the literature, in particular with those obtained using the 'on-chip' approach and similar geometries [Bliss, 2007], [Hubner, 2001]. The sensitivity reported here is currently limited by the background signal, measured in a channel filled with buffer and equal to $34 \mathrm{fW}$ (or $10000 \mathrm{cps}$ of the photomultiplier tube). It is worth noting that the fluorescence signal, corresponding to the lowest measured concentration, exceeds the background level by an amount which is seven times higher than the noise. It was originally envisioned that the limit of detection could be significantly decreased by increasing the excitation power, optimizing the quantum efficiency of the detector and reducing the background signal. During the course of this thesis, however, a well-known principle was implemented in the context of integrated optical WG excitation of fluorescence, and subsequent ultrasensitive fluorescence detection. This principle consists of modulation-frequency encoding of the excitation light sources and consequent Fourier-analytical decoding of the detected fluorescence signal, to further lower, by a factor of almost 200, the limit of detection to reach $220 \mathrm{fM}$, as will be described in detail in Chapter 4 . 


\subsubsection{Sensing of dynamic flow events}

Having illustrated the ability of the fs-laser integrated WGs to excite fluorescence in a monolithic MF channel in a spatially selective manner, in this section we explore the extension of this functionality to dynamic flow scenarios as encountered in most biochemical analytical devices. As described in chapter 1 , section 1.2, this thesis focuses on $\mathrm{CE}$ as the biochemical analysis tool. In this flow regime, electrically charged or polarized molecules introduced to a bulk capillary or a MF channel in the case of microchip CE, under an applied electric field, migrate in the direction of the oppositely charged electrode. The DNA molecules used in the context of this thesis are negatively charged owing to the presence of the phosphate groups along their chemical backbone.

The separation of analyte constituents of an analyte mixture by CE is dependent on the differential migration of analytes in an applied electric field. The electrophoretic migration velocity $(v)$ of an analyte toward the electrode of opposite charge is given by, $v=\mu E$, where $\mu$ is the electrophoretic mobility and $E$ is the electric field strength. The electrophoretic mobility is proportional to the ionic charge of a sample and inversely proportional to any frictional forces present in the fluid medium filling the MF channel. When two species in a sample have different charges or experience different frictional forces, they will separate from one another as they migrate through the MF channel. The frictional forces experienced by an analyte ion depend on the viscosity $(\eta)$ of the medium and the size and shape of the ion. Accordingly, the electrophoretic mobility of an analyte at a given $\mathrm{pH}$ is given by, $\mu=\frac{z}{6 \pi \eta r}$, where $z$ is the net charge of the analyte and $r$ is the Stokes radius of the analyte. The Stokes radius is given by, $r=\frac{k_{B} T}{6 \pi \eta D}$, where $k_{B}$ is the Boltzmann constant, and $T$ is the temperature, $D$ is the diffusion coefficient. These equations indicate that the electrophoretic mobility of the analyte is proportional to the charge of the analyte and inversely proportional to its radius. The electrophoretic mobility can be determined experimentally from the migration time and the field strength as will be described in detail in chapter 3 .

The setup with integrated optical detection described earlier was tested during a preliminary dynamic flow experiment by performing injection and electrophoresis of a 23-nucleotide, Cy3-labelled single-stranded DNA (ssDNA) molecule plug. Figure 2.18 shows two electropherograms corresponding to different ssDNA concentrations (10 $\mathrm{nM}$ and $1 \mathrm{nM}$ ). 


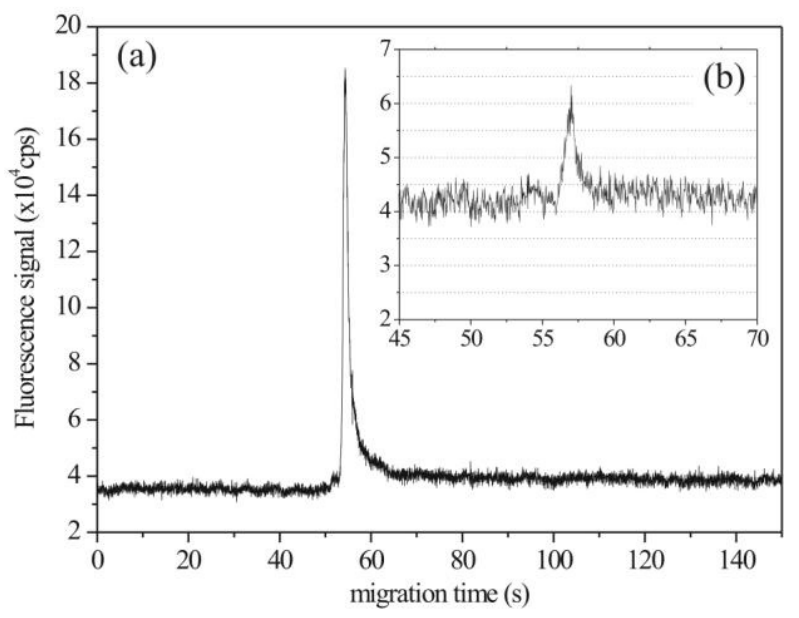

Fig. 2.18 Electrophoresis of a 23-nucleotide, Cy3-labeled ssDNA molecule plug. at a concentration of (a) $10 \mathrm{nM}$, and (b) $1 \mathrm{nM}$, in the optofluidic chip

The effective laser excitation power incident on the WG was $100 \mu \mathrm{W}$ in both cases. A quantitative estimation of the sensitivity of the device can be obtained from the signal-to-noise ratio (SNR) of the measurement [Rech, 2006], defined as $\mathrm{SNR}=\left(C_{p}-C_{B}\right) / \sigma_{B}$, where $C_{p}$ is the peak counting rate, $C_{B}$ the average background count rate, and $\sigma_{B}$ the standard deviation of the background signal. In Fig. 2.18 (b), $\sigma_{\mathrm{B}} \approx 2000 \mathrm{cps}$, so that the $S N R$ is 10, corresponding to a LOD of $250 \mathrm{pM}$. Having illustrated the integrated optical monitoring of a flowing single fluorescent species in a MF channel, the next test was naturally to exploit capillary electrophoresis for molecular separation. Therefore, as a proof of principle, a sample containing two different fluorescent dyes, Rhodamine-6G and Rhodamine-B (absorption maxima at 530 and $540 \mathrm{~nm}$, respectively) was used to demonstrate capillary electrophoresis separation in this MF chip. Further, in another, independent experiment, a sample containing double-stranded DNA (dsDNA) molecules obtained by polymerase chain reaction (PCR) [Saiki, 1988] were flown, separated, and detected by means of intercalating fluorescent dyes excited by a $488 \mathrm{~nm}$ laser beam from an Ar ion laser, in this optofluidic chip, as shown in Fig. 2.19(a). During PCR, a specific region is selected from a genetic segment (template) and amplified by means of ssDNA primer molecules end-labeled with fluorescent dye molecules. The resulting product of amplification is termed amplicon, and it is also end-labeled with the same fluorescent dye molecules present on the ssDNA primer molecules. These analyte molecules were introduced into reservoir 1 of the electrophoretic microchip (design 1) (Fig. 2.15(a)). The MF channels were filled with a 
buffer (20 mM MES / $20 \mathrm{mM}$ His, pH 6.2). Application of optimized high voltages in the range of $1-2 \mathrm{kV}$ at the MF reservoirs with integrated platinum electrodes causes the analyte molecules to flow into the $\mathrm{CE}$ injection channel from reservoir 1 to reservoir 3. By switching the voltages at all four reservoirs simultaneously to well-chosen, optimized values, a well confined plug of the mixture of the two dye molecules - with a volume of approximately 30 picoliters at the crossing junction of the two MF channels - is injected into the electrophoretic separation channel, from the MF crossing junction toward reservoir 4 . The entire on-chip flow was controlled with a LabVIEW script steering an MF control system (Capella, from Capilix BV). The $543 \mathrm{~nm}$ line from a green $\mathrm{He}-\mathrm{Ne}$ laser was coupled into the on-chip integrated WG. Again, distinct fluorescent segments appear and fade away as the two plugs migrate across the excitation WG, as shown in Fig. 2.19(b).

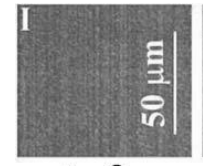

$\mathbf{t}=\mathbf{3} \mathbf{s}$

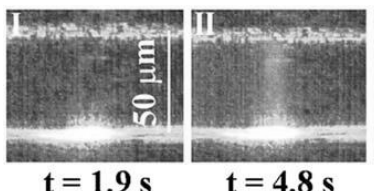

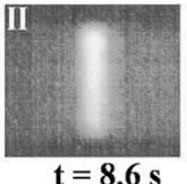

$t=8.6 s$

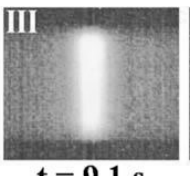

(a)

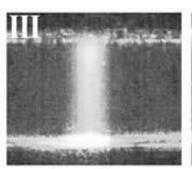

$\mathbf{t}=\mathbf{5 s}$

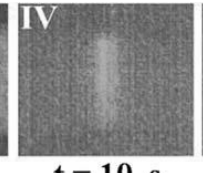

$t=10 s$

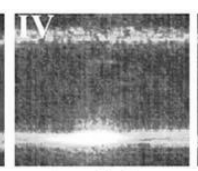

(b) $t=5.9 \mathrm{~s}$

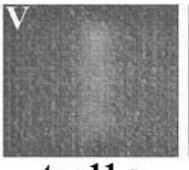

$\mathrm{t}=11 \mathrm{~s}$

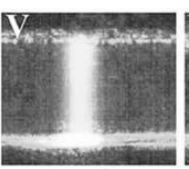

$\mathbf{t}=\mathbf{8 s}$

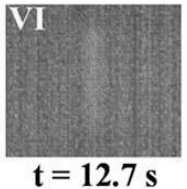

$\mathrm{t}=\mathbf{1 2 . 7 \mathrm { s }}$

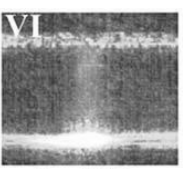

$\mathbf{t}=8.7 \mathrm{~s}$

Fig. 2.19 Passage in a MF channel, and across an excitation WG, of (a) fluorescently labeled dsDNA molecules, and (b) fluorescent dyes Rhodamine-6G and Rhodamine- $B$.

Snapshots I (before arrival of the plugs), II and III (appearance and passage of the first plug ), IV (transient period where the first plug has passed and the second plug is yet to appear), and V and VI (passage of the second plug), respectively, correspond to the significant stages during $\mathrm{CE}$ separation. In both cases (i.e., the cases of dsDNA and dye molecules), two distinct fluorescent plugs were thus observed owing to the ensuing separation during the passage. The narrow fluorescent segments in Fig. 2.19 remind us of the integrated-WG-excitation-enabled exact definition of the dimensions of the detection window (the region of intersection of the MF channel and the WG from where the fluorescence signal is collected in a perpendicular direction out of the MF chip plane) down to $12 \mu \mathrm{m}$ and its inherent 
alignment with respect to the MF channel as shown in Fig. 2.15(right). This presents an important advantage compared to conventional approaches for lowering the dimensions of the LIF detection window, e.g., the use of a pinhole (typical dimensions of $400 \mu \mathrm{m}$ ) in the optical path toward the detector [Jiang, 2000], since it achieves an inherently constant mutual alignment of the excitation and detection windows. This renders the system somewhat more compact, faster to operate, and highly reproducible. While such integration of optical excitation WGs is a first step in the more complex challenge of integrating the entire sensing functionality, it is nevertheless an important step that could potentially make the finally resulting integrated device attractive for field applications. Besides, one also needs to increase the spatial and temporal resolution with which the consecutive peaks in an electropherogram can be distinguished from each other, depending of course on the interplay with the current and the future MF protocols, as will be described in detail in chapter 3. In order to quantify this resolution, the passage of the fluorescent dyes was captured in the form of a movie, where each frame consisted of $640 \times 512$ color (RGB) pixels. A region of interest was selected to span over $80 \times 40$ pixels, with its center at the bright fluorescent segment (Fig. 2.19) corresponding to the WG - MF channel intersection, and the intensities of all pixels in this region were integrated at a time intervals of $300 \mathrm{~ms}$ to obtain the corresponding fluorescence intensity data points as a function of time for the case of the dsDNA molecules and the dye molecules, plotted as an electropherogram (Fig. 2.20).

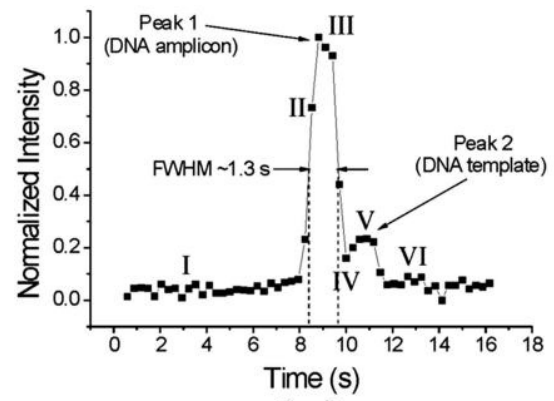

(a)

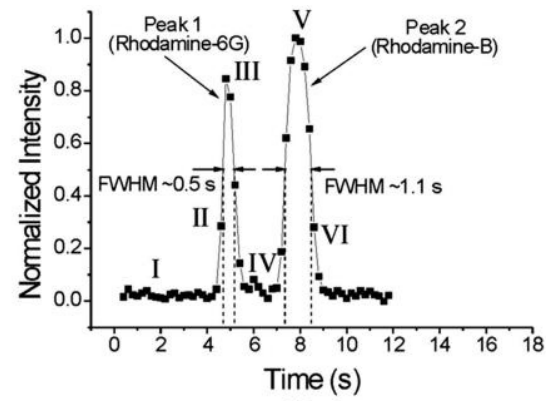

(b)

Fig. 2.20 Time-dependent fluorescence intensity at the $W G-M F$ channel intersection after injection into the separation channel for a sample containing (a) fluorescently labeled dsDNA molecules (amplicon and template) or (b) fluorescent dyes Rhodamine- $6 G$ and Rhodamine- $B$.

The two distinct peaks in Fig. 2.20(b) correspond to the electrophoretically separated Rhodamine- $6 \mathrm{G}$ and Rhodamine-B plugs, while the two peaks in Fig. 2.20(a) correspond to the electrophoretically flown dsDNA molecules. In Fig. 2.20(a), the presence of the low-intensity peak 
(appearing at time $=11 \mathrm{~s}$ ) next to the anticipated dsDNA amplicon peak (appearing at time $=9 \mathrm{~s}$ ) can be attributed to the presence of residual template material in the analyte mixture. The roman numerals (I-VI) in Fig. 2.20 denote the data points resulting from the analysis of the corresponding snapshots (I-VI) in Fig. 2.19, respectively. The peak resolution for the relatively small-sized dye molecules in Fig. 2.20(b) is more or less satisfactory (plate number [IUPAC, web] is given by $5.54 \mathrm{x}$ (migration time / peak width $)^{2}=554$ (or $\sim 180000$ per meter)). Here the low value compared to conventional CE-LIF experiments, owes more to the fact that the measurement took place only a few $\mathrm{mm}$ after the injection cross than to the real separation resolution itself. The two peaks corresponding to relatively larger dsDNA molecules in Fig. 2.20(a) are not as well resolved as one would like. The challenge to achieve a higher resolution must be addressed by means of improved MF protocols to enable high resolution $\mathrm{CE}$ separations, if one has to employ this optofluidic setup for real-world applications involving analysis of a larger number of dsDNA molecules with high resolution. This issue will be addressed in further detail in chapter 3 .

\subsection{Summary}

MF chips capable of electrophoretic biochemical analysis were fabricated by LioniX BV in fused silica glass, by making use of conventional, mature cleanroom-based technologies, keeping in mind the potential future demand of transfer to a high-volume production facility. Monolithically integrated optical WGs were micromachined into such fluidic microchips by colleagues at the Politecnico di Milano, by means of ultrafast pulses from a fs laser. This serial, but nevertheless fast (few minutes per chip) post-processing approach enabled us to exploit the existing, mature fluidic microchip fabrication techniques, while allowing for flexibility in the choice and position of optical sensing devices that ought to be integrated in such a chip. The WGs were optically characterized and were found to possess satisfactory characteristics w. r. t. mode profiles, refractive index profiles, and propagation losses. Optofluidic characterization of the chips by means of static and flowing fluorescent species (dyes as well as ssDNA and dsDNA molecules) by colleagues at the Politecnico di Milano and ourselves, respectively, further confirmed their excellent functionality. These experiments also showed the feasibility of our setup to implement diagnostically relevant dsDNA separation/analyses, upon the implementation of optimum MF protocols as described in chapter 3. 


\section{Fluorescence monitoring of electrophoretic DNA sorting}

\subsection{Introduction}

In the previous chapter, we discussed the monolithic integration of optical waveguides (WGs) for fluorescence excitation in a microfluidic (MF) chip [Martinez Vazquez, 2009]. While several micromechanical approaches have been investigated for sorting larger ( $>5$ kilo-base-pairs) double-stranded (dsDNA) molecules [Chou, 1999] [Han, 2000] [Schmidt, 2007], capillary electrophoresis (CE) [Landers, 2003] utilizing strong electric fields to separate differently sized molecules is a powerful method suitable for a broader range of molecular sizes, thanks to flexible MF protocols, exploiting a variety of channel wall coatings, sieving gel matrices, etc., and CE was therefore also the method of choice for the dsDNA analysis applications described in this thesis. Preliminary optofluidic characterization within a dynamic flow experiment as described in chapter 2, section 2.4.2, revealed the need for further optimization of our approach in order to reach the levels of electrophoretic performance close to the state of the art in microchip CE [Agilent, web] [Bio-rad, web] [Paegel, 2002] [Landers, 2003] [Herold, 2009]. While CE in general provides a potentially higher separation resolution as compared to slab-gel electrophoresis, owing to the higher electric fields that can be applied, this needs a careful optimization of the multiple MF parameters that play a role in determining the resolution. In this chapter we will describe how we addressed these optimization issues in section 3.2, while in section 3.3 we will describe the experimental results of electrophoretic dsDNA separation within the diagnostically relevant size range and the ensuing quantitative analysis. Finally, we will also comment on the performance of our system in comparison to the state of the art and the steps that can be foreseen to improve our performance in the future.

\subsection{Optimization of MF parameters}

In this section, we shall discuss the MF parameters associated with the flow of fluorescently labeled biochemical species (dsDNA molecules in the context of this work) in the MF channels in the fused-silica optofluidic microchip. The correct choice and the fine tuning of these variables played a major role in enabling us to achieve well resolved dsDNA separation, and in employing our optofluidic approach in relevant diagnostic situations. In particular, the design of the MF channels, coating of the channel walls in 
order to suppress the electro-osmotic flow (EOF), and the application of sieving gel matrices to enhance the dsDNA separation resolution will be discussed.

\subsubsection{The MF channels}

As discussed in chapter 2 (section 2.2.1), the original MF chip design 1 (made available by LioniX BV) consisted of a MF channel network involving a large number of bends to maximize the lengths of the individual channels (in order to enable full separation of relatively larger species in the analyte mixture), while maintaining a small final footprint of the chip.

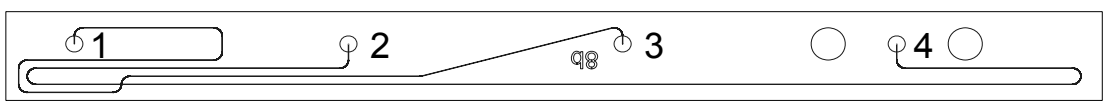

Fig. 3.1 Layout of the MF chip - design 1, with a longer sample injection channel (reservoir $1->$ reservoir 3 ), incorporating MF channels with cross-section dimensions of $50 \mu \mathrm{m} \times 12 \mu \mathrm{m}$, breadth $x$ height.

One of the approaches leading to an enhancement of the separation resolution was the decision to revise the design 1 , in order to address chiplevel issues that limit the resolution. One observation during the injection of fluorescent analyte mixures in chip design 1 was the frequent occurrence of clogging of the MF channel from reservoir 1 to reservoir 3, owing to the relatively large number (8) of bends as the channel traverses the chip. Also the relatively large length $(61.43 \mathrm{~mm})$ of this channel at times lead to premature separation of the fluorescent analytes even before the mixture was injected into the electrophoretic separation channel. The MF channel cross section dimensions ( $50 \mu \mathrm{m} \times 12 \mu \mathrm{m}$, breadth $\mathrm{x}$ height) also contributed to clogging and limited the applicability as well as the concentrations of the highly viscous, polymer-based sieving gel matrices that could be introduced in the channel network in order to enhance the separation resolution. The new chip design (design 2) was developed in order to address these issues.

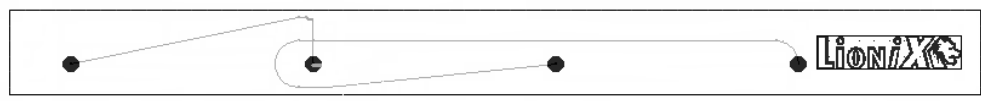

Fig. 3.2 Layout of the MF chip - design 2, with a much shorter sample injection channel (reservoir 1 -> reservoir 2) with less kinks, incorporating MF channels with cross-section dimensions of $110 \mu \mathrm{m} \times 50 \mu \mathrm{m}$, breadth $\times$ height. 
One key difference between the two designs is that the design 2 employs the MF channel from reservoir 1 to reservoir 2 to introduce the fluorescent analytes at the MF crossing junction prior to separation. The much smaller number of bends (1) and a much smaller length $(19.6 \mathrm{~mm})$ contributed to eliminating the occurrences of channel clogging, which was of practical significance. The other important difference was the much larger MF channel cross section dimensions ( $110 \mu \mathrm{m}$ x $50 \mu \mathrm{m}$, breadth $\mathrm{x}$ height), which reduced the surface to volume ratio, which also contributed to eliminating the occurrence of channel clogging. An important point is however the fact that while the functionality was improved, the footprint and the relative positions of reservoirs on the chip remained the same, meaning that the design continues to be compatible with the peripheral equipment such as the MF cartridge, useful for backward compatibility which is critical for commercial success.

\subsubsection{Suppressing EOF}

EOF is the motion of the solvent liquid induced by an applied electric potential across a bulk capillary or a MF channel in a direction opposite to the direction of the flow of the negatively charged solute molecules (DNA molecules in the context of this thesis). EOF is caused by the Coulomb force induced by an electric field on net mobile charge in a solution. Because the chemical equilibrium between a solid surface and an electrolyte solution leads to the interface acquiring a net fixed electric charge, a layer of mobile ions, known as the electrical double layer or the Debye layer, forms in the region near the interface, in this case at the interface between the MF channel inner walls and the liquid filling the channel.

When an electric field is applied to the fluid by means of the Pt electrodes integrated in the MF reservoirs, the net charge in the electrical double layer, and thereby also the fluid is induced to move by the resulting Coulomb force. EOF depends strongly on the quality of the walls of the microchannels. This implies that small defects in the walls will create "turbulences" in the EOF that will affect the capillary electrophoresis experiment by broadening the injected MF plugs [Ghosal, 2004], in addition to the broadening induced by diffusion. Therefore, in most applications of CE, e.g. in dsDNA sequencing, it is necessary to suppress the EOF [Slater, 2010] to achieve optimal separation solely by means of electrophoresis.

Typical approaches include polymer adsorption on the capillary walls [Chiari, 2000], invocation of hydrodynamic effects [Kalyakin, 2009], plasma polymerization on the MF channel walls [Salim, 2009] or the use of zwitterionic surfactants [Yeung, 1997], etc. (The polymer based approaches have already found applications in a number of commercial systems 
[Applied Biosystems, web], and a number of EOF suppression products are also commercially available [Target discovery, web]). Any adverse interaction between the analyte and the channel walls must also be prevented in order to ensure an intra- as well as inter-experimental reproducibility and to prevent clogging of MF channels [Yeung, 1997].

An effective modification of surface properties can be achieved by coating the walls of the MF channels with a specific polymer. A variety of approaches [Hovrath, 2001] have been described making use of polymer complexes based on poly(dimethylacrylamide) [Cretich, 2005], poly(ethylene oxide) [Lin, 2006], poly(vinyl alcohol) [Chiari, 1998], poly(vinyl pyrrolidone) [Kaneta, 2006], etc. Neutral polymers, chemically or physically adsorbed on the wall, strongly decrease EOF by shielding surface charge and by increasing local viscosity.

To coat the MF channels of the electrophoretic microchip we used a method based on the dynamic adsorption of a hydrophobic polymer epoxypoly-dimethyl-acrylamide (EPDMA) already used with success in other MF chips [Loughran, 2005] [Cretich, 2003] [Chiari, 2000]. Briefly, a pretreatment of the chip with a $0.1 \mathrm{M}$ solution of $\mathrm{NaOH}$ for 30 min was followed by a 30 -min treatment with a $0.6 \%$ weight / volume solution of EPDMA in $12 \% \mathrm{w} / \mathrm{v}$ ammonium sulphate. The EPDMA solution was introduced into the channel by applying vacuum to one reservoir while keeping the other three reservoirs filled with the EPDMA solution.

Monitoring the electric current flowing in the MF channel was the method [Huang, 1988] used to measure EOF in the MF channels, before and after the implementation of the coating. The functionality of the anti-electroosmosis coating was demonstrated with the help of a Phosphate $/ \mathrm{Na}^{+}$buffer solution, with $\mathrm{pH} 7.2$, at two different concentrations, 50 and $25 \mathrm{mM}$. The MF channels were filled with the higher conductivity buffer $(50 \mathrm{mM})$ applying vacuum at reservoir 4 until no air bubbles were observed. Then a voltage of $1200 \mathrm{~V}$ was applied to reservoirs 1,2 and 3 for 5 minutes to determine the baseline current. Afterwards reservoir 2 was emptied and filled with the 25 $\mathrm{mM}$ buffer, a voltage of $1000 \mathrm{~V}$ was applied for $25 \mathrm{~min}$., and the current was measured.

If EOF is present, the lower-conductivity buffer replaces the initial higher-conductivity one and a current drop is observed. By measuring the time $\tau$ required to replace the higher-conductivity buffer with the lower conductivity one, the electro-osmotic mobility $\left(\mu_{\mathrm{eo}}\right)$ is obtained as $\mu_{e o}=$ $L^{2} /(V \tau)$ [Bello, 1994], where $V$ is the applied voltage, and $L$ is the length of the MF channel, in our case $9.4 \mathrm{~cm}$. As an example, Fig. 3.3(a) reports the current measured in an uncoated microchip as a function of time, and Fig. 3.3(b) reports that of a coated one. 


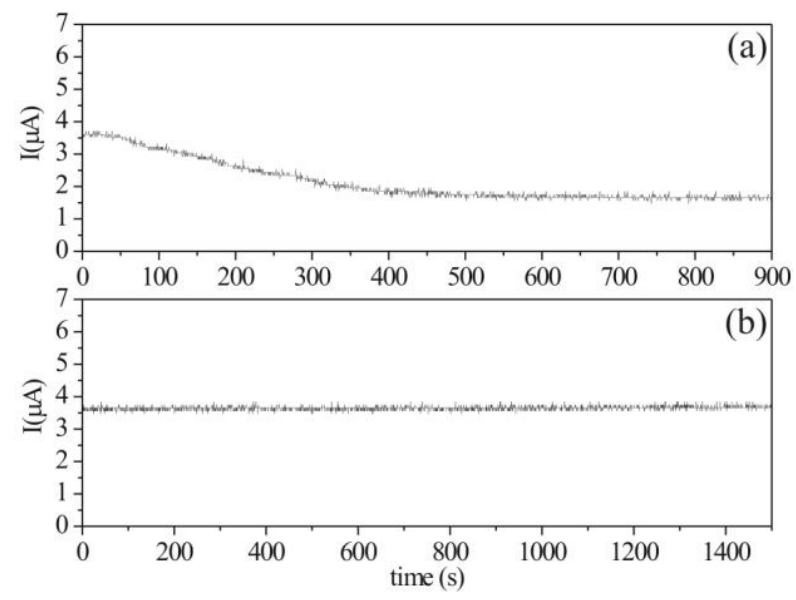

Fig. 3.3 Current monitoring experiments for EOF characterization. Current measured in (a) an uncoated and (b) a coated microchip as a function of time

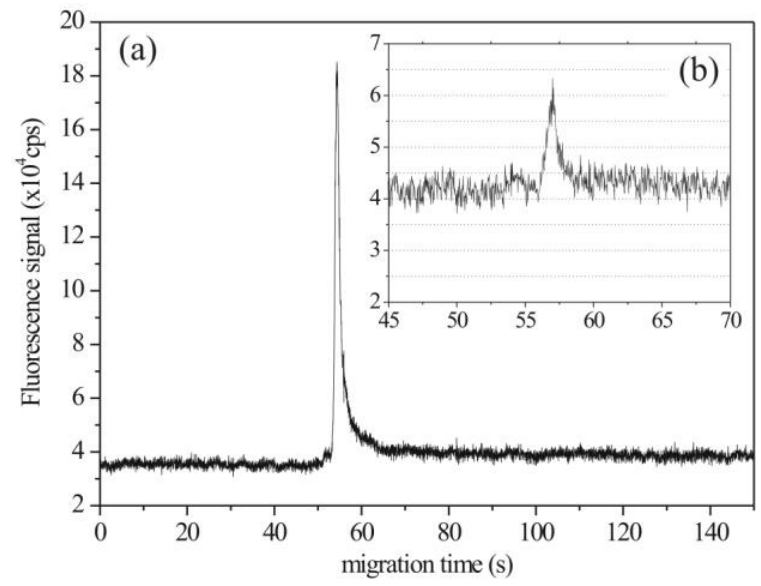

Fig. 3.4 Electropherogram of a 23-nucleotide Cy3-labeled ssDNA molecule at a concentration of a) $10 \mathrm{nM}$ and b) $1 \mathrm{nM}$, flowing in a MF channel coated with EPDMA

From the measurement in the uncoated microchip $\mu_{\mathrm{eo}}$ is estimated to be $2.5 \times 10^{-8} \mathrm{~m}^{2} \mathrm{~V}^{-1} \mathrm{~s}^{-1}$. Figure 3.3(b) shows that there is no drop in the current after 25 min., demonstrating an effective suppression of EOF on the timescale of the experiments one would typically perform in a DNA analysis diagnostic setting. A MF plug consisting of 23-nucleotide sized singlestranded DNA (ssDNA) molecules was flown in such a coated MF channel and monitored with the integrated optical WG, to obtain the electropherograms shown in Fig. 3.4. The width of the peak in the 
electropherogram allows us to estimate the length of the plug at the detection point. As the distance from the WG to the crossing point is $31 \mathrm{~mm}$, and assuming a constant velocity, we obtain a plug length of $1 \mathrm{~mm}$ when it passes in front of the WG. When the plug enters the separation channel it has about the same length as the MF channel width, i.e. $50 \mu \mathrm{m}$, thus it undergoes a significant broadening during migration. This broadening is quite standard in electrophoretic flow experiments and can be mainly attributed to diffusion [Slater, 1993] [Slater, 1995] [Gas, 2002].

Additional causes of dispersion of the plug are Joule heating [Slater, 1995] [Gas, 2002] [Wang, 2004] generated by the passage of electrical currents along the buffer and residual sample adsorption at the channel walls, which could be a potential cause of the peak asymmetry. The other cause, namely the Poissonian statistics governing the flow is discussed in section 3.3.2 later in this chapter.

The stability of the coating was assessed by repeated injections and electrophoretic flow of the ssDNA plug. It allowed 30 measurements before the EOF appeared again. Rinsing the channel with a highly concentrated $\mathrm{NaOH}$ solution $(1 \mathrm{M})$ totally removed the remaining coating. Subsequently, a second coating of the microchannel was possible and allowed one to reuse the same chip for another 30 runs. This is important for practical applications where one might use the coated channels in the MF chip often, obtaining reproducible fluidic characteristics in each coating run.

\subsubsection{Molecular sieving}

Gels and polymer matrices are commonly used to analyze dsDNA molecules. One of the most common is the agarose gel, a polysaccharide of zero net charge. The pore sizes of agarose are hundreds of nanometers and thus appropriate for separating dsDNA from the $100 \mathrm{bp}$ up to tens of $\mathrm{kbp}$. For separating smaller double-stranded DNA (dsDNA) (150-1000 bp), polyacrylamide gels are more suitable. Polyacrylamide gels are chemically crosslinked and pore sizes down to a few nanometers can be achieved by controlling the chemistry used in the gel preparation. It is generally difficult to load gels into capillaries, so entangled polymer matrices are often employed for CE. Useful reviews of such dsDNA gel electrophoresis are available in the literature [Heller, 1995] [Viovy, 2000] [Slater, 2002] [SaliebBeugelaar, 2009].

When modeling dsDNA electrophoresis in a gel or entangled polymer network, it is common to model the sieving matrix as a maze or network of pores of size $b$. The ratio of pore size and the dimensions of the dsDNA molecule are very important for the way the dsDNA molecules migrate through the gel, which is described as the three main separation regimes: (i) 
the Ogston sieving regime, where the pore size $b$ is larger than the radius of gyration $R g$ of the dsDNA molecule and the dsDNA molecules migrate through the network of pores without perturbation of their conformation. The standard model for electrophoresis in this regime is normally called Ogston sieving [Ogston, 1958]; (ii) the biased reptation regime, where $b$ is smaller than $R g$; i.e. the radius of gyration of the dsDNA molecule is larger than the nominal pore size of the gel, and the dsDNA molecule must uncoil in order to enter the pore space. The dsDNA moves through the gel like a snake, that is, "reptates" and the electrical force causes preferential motion in the direction of the electric field [Lerman, 1982] [Lumpkin, 1982]; and (iii) the entropic trapping regime, where $b$ is approximately equal to $R g$. The existence of pore dimensions comparable to the radius of gyration provides two possibilities when the molecule is pulled through the gel by the applied electrical field. First, the complete chain can be distributed among several pores (thus stretching the molecule), and second the molecule can be squeezed into one single pore. In a gel, usually, the pore size varies. This will provide a preference of the molecule for the larger pores, where the entropy loss is smallest. Squeezing the entire molecule in such a single pore will provide a minimal entropy loss, which is favored above stretching. In other words, larger pores can be seen as entropic traps [Rousseau, 1997]. Depending on the interplay of these regimes, the dsDNA molecules under analysis will have varying electrophoretic mobilities as a function of their size, which will be used for the CE separation of the individual species.

While the electrophoretic separation of dsDNA fragments is traditionally performed using rigid slab gel (acrylamide or agarose) systems, a much simpler approach has been provided by "sieving matrices" consisting of a soluble polymer added to a background electrolyte. While there has been extensive research into the appropriate choice of sieving matrix [Albarghouthi, 2000] [Brody, 2004], selection of the "best" system is not a trivial matter and depends on the application. As such, several different polymers, e.g., hydroxyethyl cellulose (HEC) [Tian, 2002], hydroxypropyl cellulose (HPC) [Sanders, 2006], agarose [Ishido, 2010], linear polyacrylamide [Salas-Solano, 1998], poly(vinyl pyrrolidone) [Gao, 1998], and poly(ethylene oxide) [Kim, 1997] have all been employed effectively for dsDNA separations. Using comparable polymer concentrations of each, it was determined that the HPC solution is almost 4-fold less viscous than HEC, based upon the experimentally obtained dynamic viscosities of the solutions (50 cP for HPC and $230 \mathrm{cP}$ for HEC, while water $\sim 1 \mathrm{cP}$ ) [Sanders, 2006]. The initial experiments performed in chip design 1 therefore employed HPC as the gel matrix of choice owing to its low viscosity, and the consequent ease of filling the small MF channels. The shift from design 1 to design 2 in turn also allowed the use of the more viscous HEC gel matrix 
buffered in Tris-borate-EDTA (TBE) that was specifically proven to enable high resolution dsDNA separations for the size range of 60-600 bp [Tian, 2002] which is of interest for the application to multiplex ligation-dependent probe amplification (MLPA) based diagnostics as will be described in chapter 5 .

\subsection{Electrophoretic dsDNA sorting}

In this section we discuss the results of electrophoretic dsDNA sorting experiments and their fluorescent monitoring, in the integrated optofluidic chip, thanks to the combination of optimized MF parameters described in section 3.2 .

\subsubsection{Experimental protocol}

Prior to the experimental runs the inner walls of the MF channel network were coated with the EPDMA-based polymer in order to suppress the EOF and to minimize adsorption of dsDNA molecules on the MF channel wall. Subsequently, the channels were filled with a sieving gel matrix consisting of HEC ( $2 \%$ wt./vol.), dissolved in $20 \mathrm{mM}$ MES / $20 \mathrm{mM}$ His buffer (pH 6.2) in order to maximize the resolution of the dsDNA CE separation. SYBR Green I (Molecular Probes Inc.) was added to the gel matrix according to the manufacturer's protocol [Invitrogen, web]. Prior to use, all the concerned chemicals were sterilized, and filtered $(0.22 \mu \mathrm{m}$ pore size $)$ and stored at 269 $\mathrm{K}$ after use. The CE sample loading and separation protocol was based on actuation voltages of up to $1.5 \mathrm{kV}$, delivered by Pt electrodes integrated into the MF reservoirs, with the help of a Labview (National Instruments, Inc.) program to steer the MF control system (Capella, CapiliX BV) [Capilix, web].

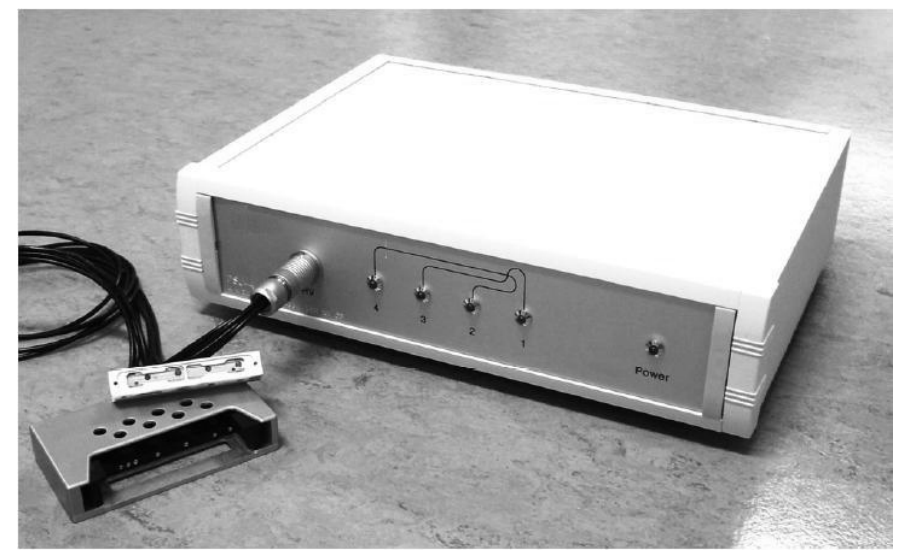

Fig. 3.5 Picture of the capilix system for flow control in the optofluidic chip 
Application of a high voltage (e.g. $1: 100 \mathrm{~V}, 2: 400 \mathrm{~V}, 3: 100 \mathrm{~V}, 4: 0$ $\mathrm{V}$ ) forces the negatively charged dsDNA molecules to migrate into the $\mathrm{CE}$ injection channel from sample reservoir 1 to waste reservoir 2. By switching the voltages at all four reservoirs simultaneously (e.g. Separation 1:975 V, 2 $: 1000 \mathrm{~V}, 3: 0 \mathrm{~V}, 4: 1500 \mathrm{~V})$ a well-confined plug of dsDNA molecules with a volume of $\sim 605$ picoliters at the crossing junction of the two MF channels - is injected into the CE separation channel, from the MF crossing junction toward waste reservoir 4 , and consequently the dsDNA molecules contained in the plug volume are electrophoretically separated according to their size.

\subsubsection{Fluorescence monitoring results and analysis}

In our integrated optofluidic chip, a commercial dsDNA ladder (D3812, Sigma-Aldrich) consisting of molecules with 17 different sizes $(50 \mathrm{bp}, 100$ bp, 150 bp, 200 bp, 250 bp, 300 bp, 350 bp, 400 bp, 450 bp, 500 bp, 600 bp, $700 \mathrm{bp}, 800 \mathrm{bp}, 900 \mathrm{bp}, 1000 \mathrm{bp}, 2000 \mathrm{bp}$, and $3000 \mathrm{bp}$ ) is injected, separated, and detected by use of an intercalating fluorescent dye. We recorded a movie (Fig. 3.6(bottom)) with a CCD camera to visualize the flow of separated dsDNA plugs across the excitation WG. While the plug migration along the MF channel is visualized by background $\mathrm{Hg}$ arc lamp illumination, a 12- $\mu$ m-narrow segment lights up when a plug crosses the intersection point with the integrated WG of corresponding width.

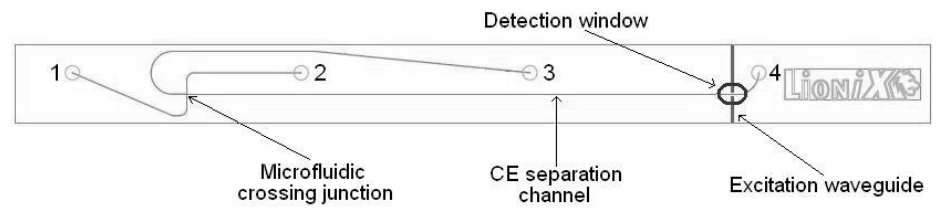

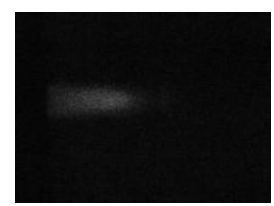

$\mathrm{t}=137 \mathrm{~s}$

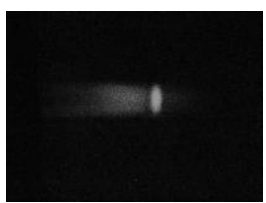

$\mathrm{t}=137.5 \mathrm{~s}$

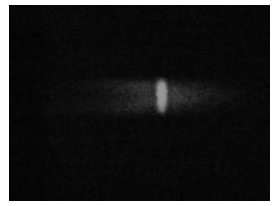

$\mathrm{t}=137.8 \mathrm{~s}$

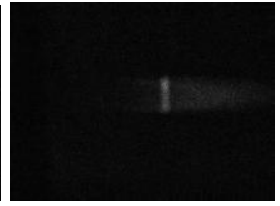

$\mathrm{t}=138.2 \mathrm{~s}$

Fig. 3.6. (top) Schematic of the optofluidic chip showing reservoirs 1-4, sample injection channel (reservoir $1 \rightarrow$ reservoir 2 ) and CE separation channel (reservoir $3 \rightarrow$ reservoir 4), as well as the integrated optical WG and detection window; (bottom) snapshots of a movie recorded with a CCD camera showing transient fluorescence from several molecule plugs formed by CE separation of a dsDNA ladder consisting of molecules with 17 different sizes (50-3000 base-pairs), fluorescence-labeled with an intercalating dye, as these plugs pass by the point of integrated-WG laser excitation at $488 \mathrm{~nm}$ 
Replacing the CCD camera by an ultrasensitive photomultiplier tube (PMT) allows us to record the fluorescence intensity vs. migration time. An example of two electropherograms measured in the same chip with integrated-WG laser excitation, in two different experimental runs is shown in Fig. 3.7. Indeed, one can see that the use of optimum MF channel wall coating and sieving gel matrix has lead to excellent CE separation of the dsDNA molecules under integrated-WG laser excitation.

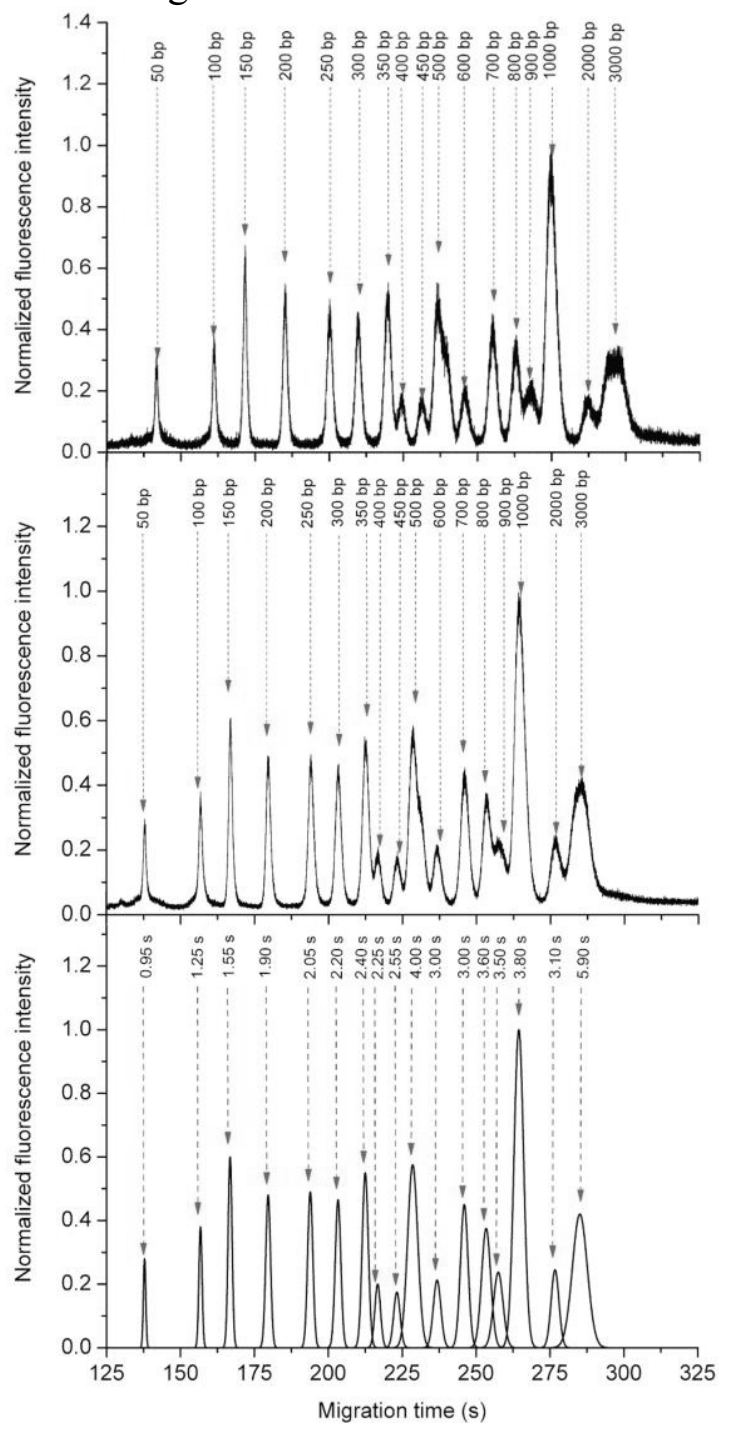

Fig. 3.7 (top and middle) Normalized fluorescence intensity vs. migration time with integrated-WG laser excitation (bottom) An example demonstrating Gaussian fits to the peaks in the middle electropherogram; the peak widths are expressed as full-width-at-halfmaximum 
Based on the ratio of the maximum fluorescence signal to the standard deviation of the background noise, we estimate a LOD of $\sim 2.1 \mathrm{pM}$, surpassing previously reported LOD values in laboratory setups involving on-chip-integrated fluorescence monitoring [Rech, 2006] [Bliss, 2007] [Bliss, 2008] [Martinez Vazquez, 2009]. The definition of the LOD [Vogelgesang, 1987] given by the four-fold standard deviation of the noise was used in our evaluation (an injected dsDNA concentration of $10 \mathrm{nM}$ diluted by, firstly, the spatial separation of 17 different molecule sizes and, secondly, plug broadening from $110 \mu \mathrm{m}$ to at least $248 \mu \mathrm{m}$, provided a fluorescence signal which is 125 times the four-fold standard deviation). There is however still room for improving our current value of the intrinsic LOD of the setup. Even lower values have been reported in a number of bulk optical, confocal LIF setups, which claim even down to single-molecule detection [Foquet, 2002] with LODs as low as $300 \mathrm{fM}$ [Johnson, 2004]. In chapter 4, we present a technique to further minimize the LOD of our system by implementing Fourier filtering.

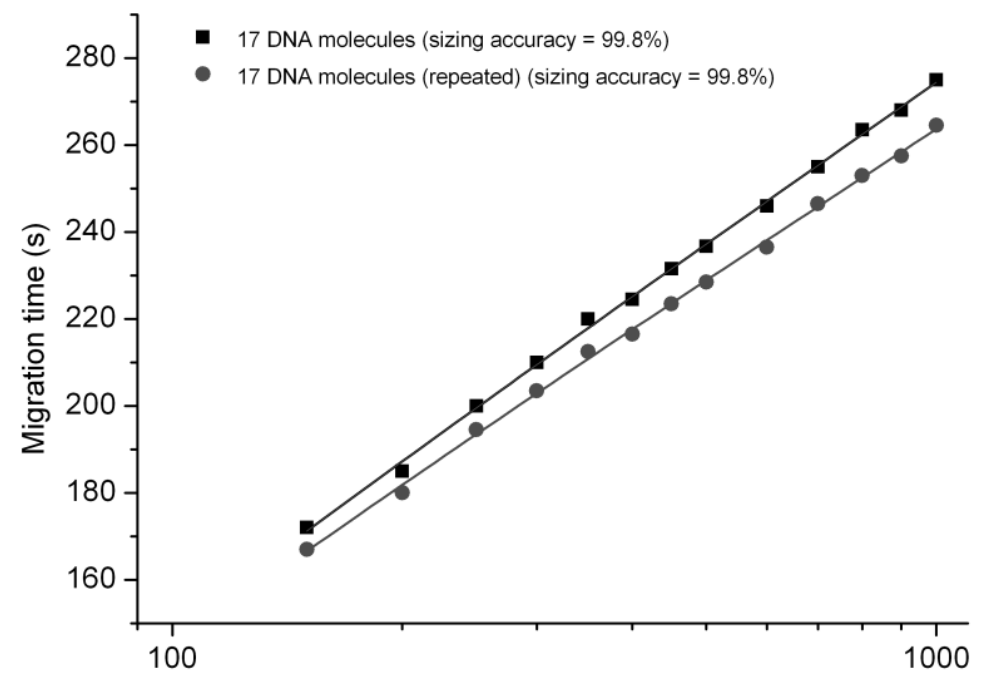

DNA size (bp)

Fig. 3.8 Migration time vs. base-pair size for the two measured electropherograms in Fig. 3.7

When plotting the temporal occurrence of the fluorescence peaks vs. their a priori known base-pair sizes, the two smallest as well as the two largest dsDNA molecules exhibit clear deviations owing to their insufficient interaction with the sieving matrix and tendency to fold, respectively, in accordance with the literature describing the sieving mechanisms for dsDNA separation by CE (conventional bulk capillary as well as in microchips) in polymeric gel matrices [Sartori, 2003] [Deutsch, 1989] [Liu, 2007] [Jung, 
2002] [Slater, 2003] and as has been described earlier in section 3.2.3. Nevertheless, the peaks of all dsDNA molecules within sizes of 150-1000 bp (Fig. 3.8), in the diagnostically relevant region as applicable for the envisioned analysis of MLPA dsDNA-probes (with typical sizes in the range 150-500 bp), are resolved with a sizing accuracy of greater than $99 \%$, when using the dsDNA sizing techniques commonplace in conventional CE-based analyses [Fundador, 2008] [Akbari, 2002]. This result ( $>99 \%$ sizing accuracy) is reproducible from experiment to experiment - as shown in Fig. 3.8 , and also from the analysis of the two experiments with an independent set of 12 labeled dsDNA molecules described in chapter 5 - and approaching the performance of state-of-the-art microchip CE without integrated-WG laser excitation [Paegel, 2003].

From the measured migration time $t$ vs. dsDNA size of Fig. 3.8 we derive the electrophoretic mobility (right-hand ordinate) according to the formula, $\mu=\frac{L}{E t}$, where $L$ is the distance between the MF crossing junction and the detection window and $E$ is the electric field strength in the MF CE separation channel. The derived linear decrease (on logarithmic scale) of the electrophoretic mobility with the logarithm of increasing dsDNA size (Fig. 3.9) matches well with results in conventional dsDNA gel electrophoresis setups [Dolnik, 1999].

After fitting the fluorescence intensity peaks (Fig. 3.7 (middle)) [Gas, 2002] [Sun, 2007] with Gaussian profiles (Fig. 3.7(bottom)) to calculate the full widths at half maximum of the Gaussian distribution, the resulting peak widths are plotted vs. the square root of the dsDNA molecule sizes (Fig. 3.10) for both the experiments (although Fig. 3.7(bottom) only exemplifies a single measured electropherogram). The square-root dependence, confirmed by the linear fit, indicates that the migration of dsDNA molecules follows Poisson statistics, the dispersion being governed by the number of collisions a molecule typically undergoes as it traverses through the sieving gel matrix [Krawczyk, 2002] [Popelka, 1999]. 


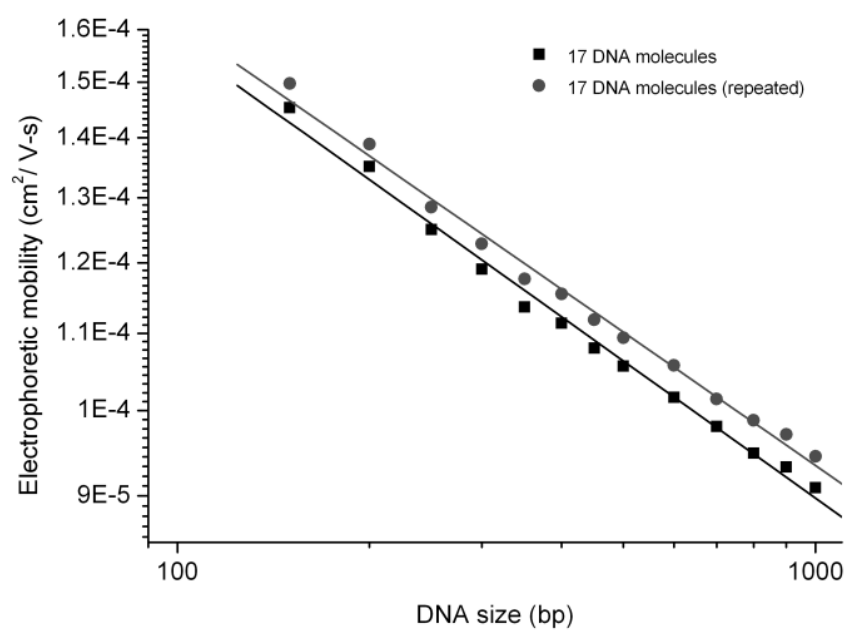

Fig. 3.9 Electrophoretic mobilities for the two experimental runs with 17 dsDNA ladder molecules (Fig. 3.7) plotted against the dsDNA size on a double-logarithmic scale

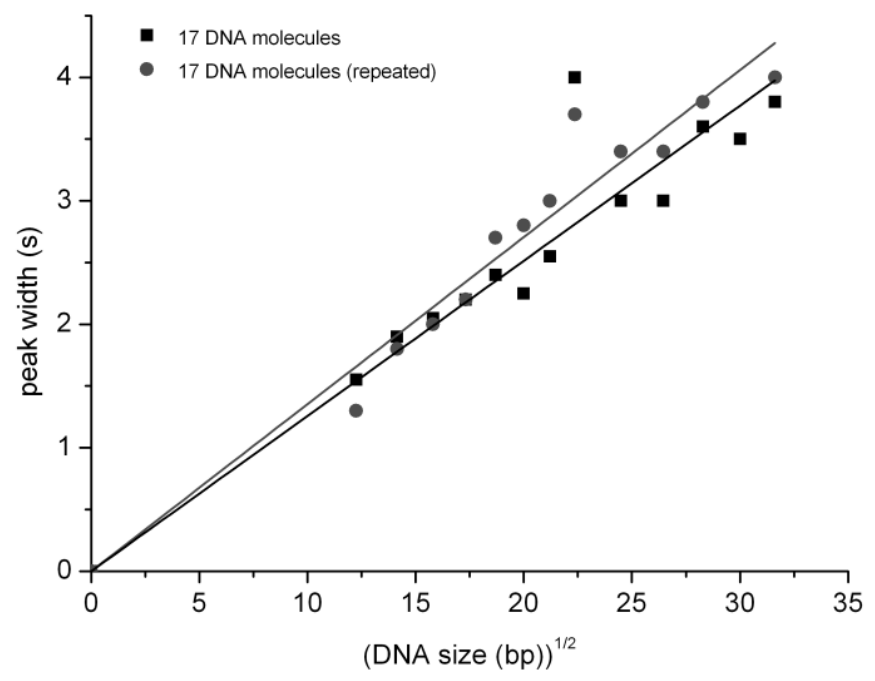

Fig. 3.10 peak widths obtained from the Gaussian fit vs. square root of the base-pair size for the two experimentally measured electropherograms of Fig. 3.7.

\subsubsection{CE separation resolution}

The major contributor to peak broadening in CE separation experiments is the longitudinal diffusion of the analyte molecule MF plugs in the capillary [Jorgenson, 1983] [Terabe, 1989]. The quantity most commonly used to describe the separation efficiency, i.e. the number of distinctly separated electropherogram peaks that can be obtained using a certain separation 
system, is the plate number $(N)$, defined as $N=\left(\frac{t}{\sigma}\right)^{2}$, where $t$ is the migration time corresponding to the electropherogram peak of a separated species, and $\sigma$ is the standard deviation of the Gaussian peak. Taking into account the relation between $\sigma$ and the full-width-at-half-maximum (FWHM) for a Gaussian function, the above can be rewritten as $N=8 \ln 2\left(\frac{t}{W}\right)^{2}=5.54\left(\frac{t}{W}\right)^{2}$, where $\mathrm{W}$ is the full-width-at-half-maximum of the peak, which is also the IUPAC definition of plate number in the context of $\mathrm{CE}$ experiments as described in this chapter [IUPAC, web]. Theoretically, the plate number is defined as the analytical expression, $N=\frac{\mu E L}{2 D}[$ Beckman Coulter 2, web], where $\mu$ is the electrophoretic mobility, $D$ is the diffusion coefficient of the solute in the separation buffer system, and $L$ is the effective length of the separation channel.

Using $\mu=\frac{L}{E t}$, we obtain $N=\frac{L^{2}}{2 D t}$. However, $\frac{L}{t}=v$ is the flow velocity, which is constant for a particular species during a certain $\mathrm{CE}$ experiment. Therefore, the quantity $\frac{N}{L}$, or plate number per unit length, provides insight into the diffusion of the species, thereby also into the peak broadening which plays an important role in the resolution of the system.

Applying the expression $N=5.54\left(\frac{t}{W}\right)^{2}$ to the experimental results described in the previous section 3.3.2, we estimate plate numbers up to 72000 per $3.6 \mathrm{~cm}$ of the separation channel that has been used for the measurements. In order to compare this performance to other $\mathrm{CE}$ experiments presented in the literature, we express the plate number per meter of separation channel. This leads to values of up to 2 million theoretical plates per meter for our experiments.

This is nevertheless quite low compared to the very high plate numbers demonstrated in off-chip bulk capillary electrophoretic separations while microchip CE was still in its early days, e.g. plate numbers higher than 30 million per meter for oligonucleotide separation using a polyacrylamide gel in a bulk fused silica capillary [Guttman, 1990] or plate numbers higher than 4 million with single bp resolution in 51-267 bp size range [Pariat, 1993]. It is evident that further optimization of the MF setup is mandatory in order to compete with traditional bulk $\mathrm{CE}$ techniques where possibly longer 
separation channels have been used to achieve higher resolution nevertheless at the expense of longer separation times.

Various techniques have been suggested in the literature for improving the separation resolution of dsDNA fragments. Higher applied electric field and lower analyte diffusion coefficient would result in higher resolutions indicated by higher plate numbers. One of the limiting factors in increasing the resolution by means of higher applied electric fields is the heat generated (Joule heating) from the power applied $(P=V . I)$ to the narrow bore capillary [Nelson, 1989]. Because of the sensitivity of the electrophoretic mobility to temperature $\left(\sim 2 \%\right.$ per $\left.{ }^{\circ} \mathrm{C}\right)$, good temperature control is extremely important for achieving good migration reproducibility and high resolution [Nelson, 1989] [Karger, 1989]. Increasing the resolution during CE separation has been reported by using different temperatures during electrophoresis [Guttman, 1991]. Migration times decrease with increasing temperature in the isoelectrostatic (constant voltage) separation mode and maximize in the isorheic (constant current) separation mode. The resolution between the short dsDNA fragments $(<300 \mathrm{bp})$ decreases in the isoelectrostatic separation mode and shows maxima in the isorheic (constant flow) mode at elevated temperature. However, the resolution in the higher size ranges $(>1000 \mathrm{bp})$ decreases in both modes with increasing temperature.

Resolution and analysis time can also be optimized by manipulation of the applied electric field. Methods based on pulsed-field electrophoresis [Sudor, 1994] [Kim, 1994], analyte velocity modulation [Demana, 1991], and field-strength gradient separation techniques [Guttman, 1992] have been reported. Another approach to improved resolution would be the optimization and use of improved geometries of the MF channel with different concentrations of various possible low-viscosity gel matrices, e.g. polyacrylamides, filling the separation channels. In this context, a lot of lessons can still be learnt from the bulk CE approaches and implemented in the MF setup for bringing its performance ever closer to the state of the art in bulk CE based apparatus. For example, bulk CE apparatus has been used in the human genome project and in other related work to identify a DNA sequence down to a single base. Detection of a single mutation, i.e. mutual replacements of a single nucleic acid bases $-\mathrm{A}, \mathrm{C}, \mathrm{G}$, and $\mathrm{T}$, requires much higher accuracies and consequently higher peak resolution than what one needs to detect only insertions and deletions of down to a few bp (as we can currently hope to achieve for total dsDNA sizes of a few hundred bp with our sizing accuracy of 99\%). It should however not be forgotten that the main drive for shifting from bulk $\mathrm{CE}$ to microchip $\mathrm{CE}$, and especially with integrated WGs is the low sample consumption, short analysis times, and the move toward potential complete integration of the MF and optical monitoring functionalities in the future MF CE chip generations. 
Each measured fluorescence peak in our measured electropherogram is a convolution - along the flow axis - of the Gaussian WG mode-field crosssection $\Delta x_{W G}$ and the diffusion-induced physical width $\Delta x_{M F}$ of the MF plug. The resulting spatial plug widths $\Delta x$ are related to the temporal electropherogram peak widths $\Delta t$ (provided in Fig. 3.10 as full width at half maximum) as

$$
\Delta x=\frac{L}{t} \Delta t=\sqrt{\Delta x_{W G}^{2}+\Delta x_{M F}^{2}},
$$

where $L=3.6 \mathrm{~cm}$ and $t$ (data on the abscissa of Fig. 3.7) are the migration length and time, respectively. In our experiment, the minimum electropherogram peak width is found to be $\Delta x=248 \mu \mathrm{m}$ (the very first peak). With $\Delta x_{W G}=12 \mu \mathrm{m}$, we obtain $\Delta x_{M F}=247.7 \mu \mathrm{m}>\Delta x_{W G}$, i.e., the current spatial resolution is limited by the MF plug width, while the obtained integrated-WG mode profile would allow for a 20 -fold better resolution. This would correspond to plate numbers as high as $20 \times 2=40$ million per meter. With such high resolutions, also a correspondingly higher accuracy can be expected. This will extend the single bp sizing accuracy even to dsDNA molecules having sizes up to $1000 \mathrm{bp}$ or higher. Highly accurate calibration for detecting bp insertions and deletions will thereby become possible also for large sized dsDNA molecules of diagnostic relevance (e.g. the MLPA probe dsDNA molecules). Also, a 20-fold increase in resolution corresponds to the ability to detect and unambiguously identify a consequently larger number of peaks within the same time scale on an electropherogram. This corresponds to the analysis of a larger number of dsDNA molecules in a single run. MF technological advances, e.g. improved MF channel-wall coatings and sieving gel matrices, may lead to smaller MF plug widths, thereby fully exploiting the monitoring resolution achieved with integrated optical WGs. Also, due to the flexibility in choosing the dimensions of the optical WGs by means of the different fabrication technologies, even further advances in resolution can be foreseen. Integrated optics is indeed well suited to handle the resolution demands of future MF-chip generations.

\subsection{Summary}

Electrophoretic flow experiments with a higher complexity (as compared with the optofluidic test experiments described in chapter 2) were performed in the integrated optofluidic chip. Various MF parameters such as dimensions and layout of the MF channels, EOF control, and dsDNA molecular sieving by means of gel matrices were described based on the 
protocols reported in the literature. In the optimized setting, a commercial dsDNA ladder consisting of 17 dsDNA molecules in the diagnostically relevant size range, but with predefined known sizes was electrophoretically separated into its individual components. Ultrasensitive fluorescence monitoring (by means of a PMT) as well as visualization (by means of a CCD camera) was implemented, and a low LOD and a high separation resolution was achieved, among others thanks to the narrow excitation window defined by the integrated optical WG. The results were analyzed w. r. t. quantitative parameters such as migration times, electrophoretic mobility, and MF plug width. A sizing accuracy of up to $99 \%$ was observed, and could potentially be used for calibrating unknown dsDNA samples in the size range of diagnostically relevant dsDNA molecules obtained by MLPA, and also for accurate simultaneous analysis of multiple MLPA samples in the multiplex setting as described in chapter 5 of this thesis. The variation of electrophoretic mobility and of plug width as a function of dsDNA size was found to be in good agreement with conventional electrophoretic analysis techniques, placing our system closely at par with the state of the art in terms of MF performance, while also pointing toward the fact that further improvements in resolution are mandatory in order to compete with the bulk $\mathrm{CE}$ setups. The current status of our setup nevertheless enables us to analyze the diagnostically relevant dsDNA separations as will be described in chapter 5. There is still room for improvement of the sensitivity and of our LOD of $2.1 \mathrm{pM}$, w. r. t. the bulk optical confocal LIF setups that report detection limits down to a single molecule. Our value of LOD however is better than the values reported in literature as far as integrated optical WGs were used for LIF monitoring during an electrophoretic flow experiment. However, while these experiments were carried out using intercalating dyes, the detection of extremely small quantities of end-labeled dsDNA molecules essential for the envisioned diagnostic application - sets even further stringency on the LOD, as will be addressed in the next chapter. 


\section{Toward ultrasensitive fluorescence detection}

\subsection{Introduction}

In the previous chapter we discussed the optimization of microfluidic (MF) parameters, which enabled us to perform the capillary electrophoresis (CE) separation and quantitative analysis of dsDNA molecules in the diagnostically relevant size range. Sensitive fluorescence detection in an optofluidic chip lead to a limit of detection (LOD) of $2.1 \mathrm{pM}$, when intercalating dye molecules were used. Intercalating dye molecules are added to the sieving gel matrix filling the MF channel during analysis, and they are not specifically selective to certain dsDNA molecules depending on their origin, as would be required by our envisioned multi-color analysis, described in chapter 5 . Even if one adds differently colored intercalating dye molecules to the different sample mixtures to potentially ensure selectivity, their attachment and detachment to the dsDNA molecules is transitory and on a millisecond time scale, i.e. much faster than the duration of our typical CE flow experiments [Daniel, 2000] [Zipper, 2004] [Lei, 1969] [Biver, 2005]. This implies that during the flow, the intercalating molecules could attach themselves to both types of dsDNA molecules under consideration for multiplex analysis. As a result, specific, exclusive labeling is not possible, as also pointed out in a number of commercial kits utilizing intercalating dyes [Applied Biosystems, web]. Exclusive labeling would have to be based on covalent end-labeling, and not intercalation [Olson, 2004]. This implies that only 1 or 2 (in case they are attached to both ends) dye molecules can be placed on each dsDNA molecule. Sensitivities must be as high as possible if such exclusively labeled samples are to be detected, and therefore reaching lower noise levels is an issue.

In this chapter we have collected all the measures taken to increase the sensitivity. In section 4.2 , we will discuss various steps taken to optimize the experimental setup. Section 4.3 focuses on the numerical implementation of lock-in amplification by means of Fourier analysis of the detected fluorescence signal, resulting in a 10 -fold enhancement of the signal-to-noise ratio (SNR), and thereby improving the LOD by the same amount. The allnumerical approach described here avoids the addition of any physical instruments to the existing experimental setup, and performs the entire processing simply with a PC. 


\subsection{Optimization of the experimental setup}

In this section we shall discuss the various approaches that were applied for increasing the sensitivity of the setup. These include the various steps taken to prepare the chip for the experiment, carefully choosing the wavelength filters to pass only the desired fluorescence signals through to the detector, etc. Before starting to work with the MF chip, its back-surface (the surface on the side of the MF reservoirs, i.e., the surface opposite to the surface facing the detector) was painted black with a permanent ink. By sealing the MF reservoirs prior to this step we made sure that no ink particles or droplets could enter the MF channels through the reservoirs, thereby clogging the chip or contaminating the analyte or the buffer solutions. Almost all the stray light that may be originating from, e.g., autofluorescence of the polymeric MF cartridge was absorbed by the black ink, thereby reducing the background noise otherwise added to the fluorescence emitted by the analyte molecules.

A multiband filter set (XF57 from Omega Optical, Inc.) [Omega, web] was used to pass only the wavelength bands corresponding to the excitation spectra of the fluorescent dyes in question. Figure. 4.1 (a-c) shows the absorption and fluorescence emission spectra of the dye molecules Alexa Fluor 488 (top), Alexa Fluor 546 (middle), and Alexa Fluor 647 (bottom), superimposed on the transmission spectrum of the filter. Only these dye molecules, and spectrally equivalent ones, were used in the experiments described in this thesis.

The well-enclosed optical path within the inverted microscope ensures once more that no stray signal interferes with the fluorescence signal from the analyte. The photomultiplier tube (PMT) was fitted directly via a Cmount adapter to the eyepiece of the microscope in order to capture the fluorescence photons released from the microscope eyepiece. The housing of the setup ensured an optimum, completely closed measurement system, with the measurements taking place in complete darkness (with all the possible sources of light including LED displays on power-meters, PC-monitors, etc, in the laboratory being either turned off or covered with black paper). 

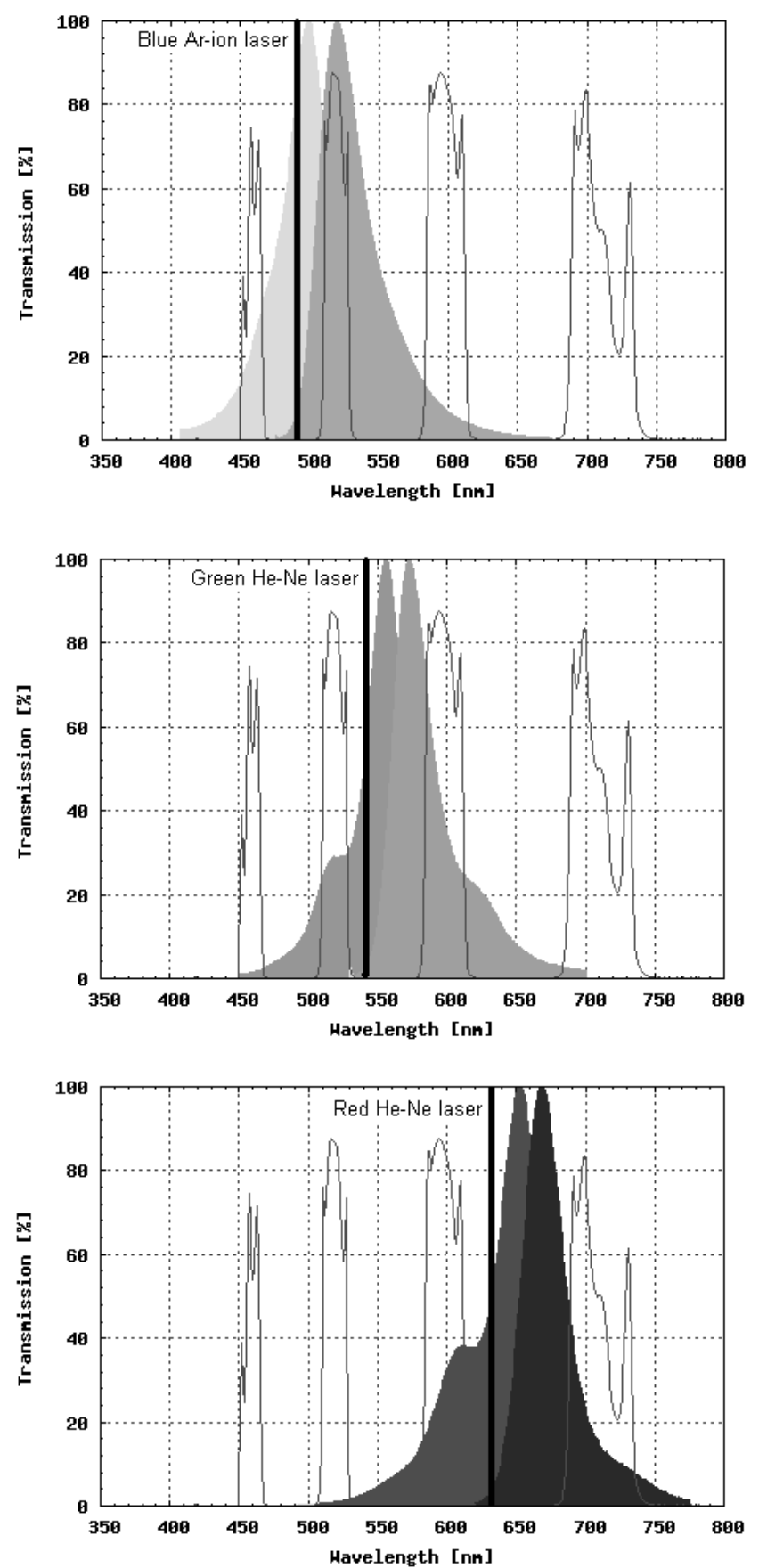

Fig. 4.1 Transmission spectrum of the multi-band filter-set XF57 (solid curves) superimposed on the absorption and fluorescence emission spectra of Alexa Fluor 488(top), Alexa Fluor 546 (middle), and Alexa Fluor 647 (bottom) [Omega optical, web] 


\subsubsection{Integrated optical excitation as opposed to Hg-lamp}

A large number of conventional fluorescence monitoring systems described in the literature utilize broadband excitation light sources such as a $\mathrm{Hg}$-arc lamp [Lin, 2001] [Kang, 2006] [Fredlake, 2008] [Lin, 2008], a Xe-lamp [Gong, 2006] [Witek, 2006] [Park, 2007], a halogen lamp [Ceriotti, 2003] [Li, 2004] [Fleger, 2004] [Malcik, 2007], etc., for diagnostic and DNA sequencing applications, and most fluorescence microscopes available on the market [Leica, web] [Olympus, web] are sold with a coupled broadband light source. Figure 4.2 depicts the emission spectrum of such an example, namely the Hg-arc lamp.

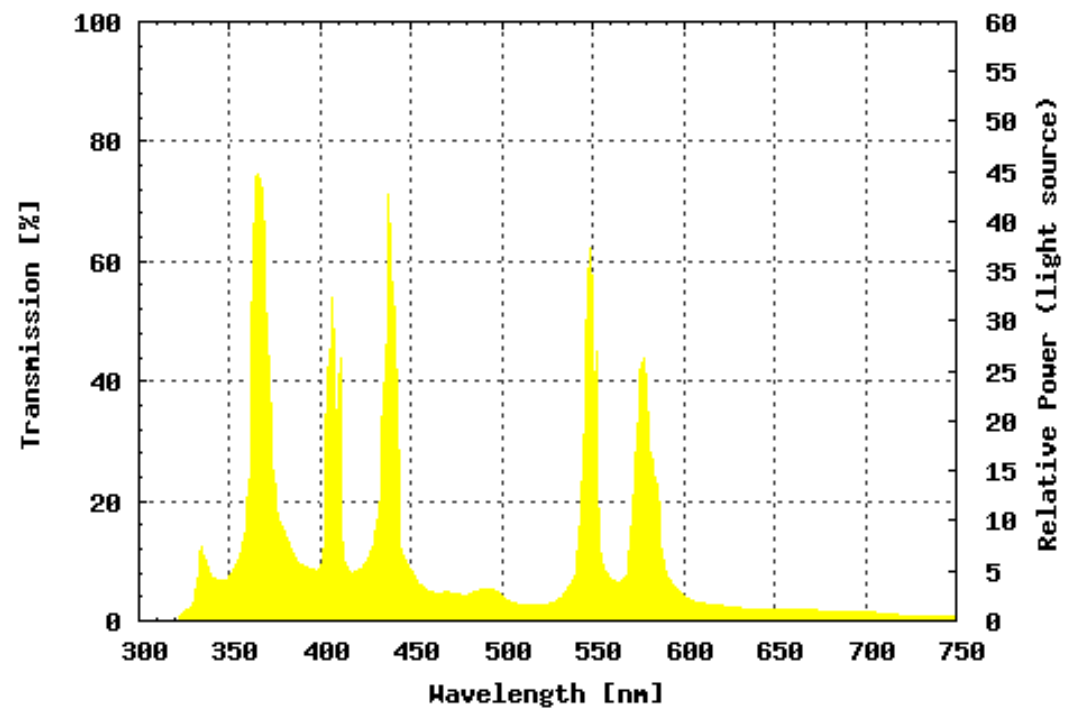

Fig. 4.2 Emission spectrum of the Hg arc lamp [Omega optical, web]

The presence of multiple emission peaks distributed along the entire visible spectrum is clearly seen, and it immediately becomes clear why it is indeed attractive to use such a light source for the purpose of fluorescence excitation. One can easily select a certain single peak or a set of peaks via an excitation filter and illuminate the entire chip for monitoring the on-chip events or focus the beam of light after filtering onto a tiny spot in a microchip where a fluorescently labeled analyte molecule is to be studied.

However, one drawback of such a broadband light source for fluorescence excitation as opposed to a narrowband laser is the relatively higher background signal, resulting from the spectral regions that are not filtered out - in spite of using high-quality filters which nevertheless have a limited extinction coefficient - and that spectrally overlap with the fluorescence emission from the analyte molecules. In addition to the spectral non- 
specificity, the excitation is also spatially non-selective. It is hard to achieve a $12 \mu \mathrm{m}$ spot (as easily obtained by integrated WG excitation) by focusing a $\mathrm{Hg}$ arc lamp using standard optical equipment. The beam waist of the Hg-arc lamp light beam painstakingly focused by the microscope's objective lens could nevertheless diverge within the distance between the objective and the sample. By using a narrow linewidth laser beam, coupled and well-confined through an integrated optical waveguide, or through a free space, bulk optical focusing system as in a confocal microscopy LIF setting, these drawbacks are overcome.

It is seen from a large number of publications in the electrophoresisrelated literature, the alternative approach to the use of a broadband lamp as the light source is the use of a focused laser beam as the fluorescence excitation light source in a confocal LIF setting [Jiang, 2000] [Lundqvist, 2003] [Obeid, 2003] [Johnson, 2004] [Kuswandi, 2007] [Fundador, 2008]. This approach has been used to achieve very low detection limits down to 10 $\mathrm{nM}$ for fluorescently labeled proteins [Fruetel, 2005], $5 \mathrm{nM}$ for Rhodamine B dye [Shen, 2009], 9 pM for Cy-5 dye [Jiang, 2000], 1.1 pM for Fluorescein [Fu, 2006], and $250 \mathrm{fM}$ for Fluorescein-isothiocyanate labeled glutamates [Lundqvist, 2003], to cite a few examples. However, the advantage of a low LOD in a confocal LIF setup is nevertheless challenged by the need for bulk optical peripheral equipment, which would be a hurdle in the advanced stages of system integration for field applications. The integrated optical WG approach as described in this thesis however already takes the first step toward such system integration and solving a part of the bigger, more complex problem, namely by integrating the laser-induced fluorescence excitation and removing the need for bulky excitation setups.

Figure 4.3 (top) shows the CE separation of 17 dsDNA molecules from a conventional dsDNA ladder (as described earlier in chapter 3), with fluorescence excitation only by a Hg-arc lamp. Monitoring instead by means of single-wavelength laser excitation of fluorescence, in a narrow $(12 \mu \mathrm{m})$ segment (as described already in chapters $2 \& 3$ ) leads to a reduction of the background noise by an order of magnitude compared to the Hg-lamp monitoring, as seen from Fig. 4.3 (bottom). 

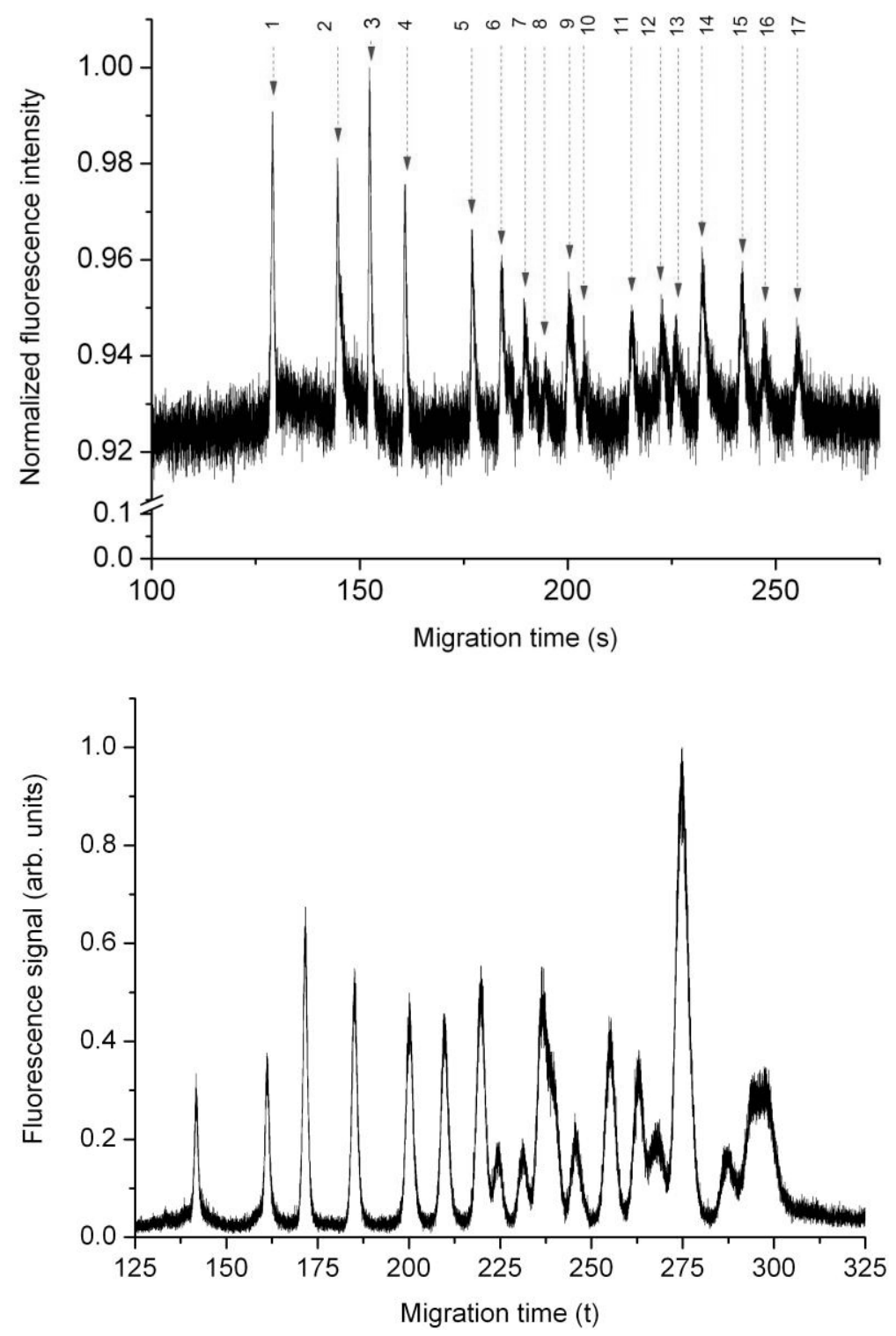

Fig. 4.3 Electropherograms depicting the CE separation of 17 fluorescently labeled dsDNA molecules from a dsDNA ladder; measured with (top) a Hg arc lamp and (bottom) integrated optical WG laser excitation

Note that in this experiment the peak width is determined mainly by the width of the MF plugs themselves and not by the width of the excitation beam, as described earlier in section 3.3 .2 of chapter 3 ; this is the reason why the difference in the spatial resolution obtainable by the integrated WG excitation as opposed to $\mathrm{Hg}$ arc lamp excitation is not directly visible in Fig. 4.3 . 


\subsubsection{MF sample stacking}

In this section we present yet another technique to increase the sensitivity of the fluorescence detection platform. Unlike the previous section 4.2.1, where the value addition of integrated optics was the key message, in this section we describe a purely MF approach based on the local increase (at the MF crossing junction) of sample concentration, just prior to commencing the $\mathrm{CE}$ separation, by means of repeated injections, back-flow, and consequent build-up of fluorescent analyte molecules at the crossing junction. Such a procedure, termed stacking [Jacobson, 2005] is illustrated below, by using a high concentration of flowing dsDNA molecules labeled with an intercalating dye filling the MF channel enabled the filming of this procedure by a CCD camera. Figure 4.4 consists of a series of snapshots obtained from this movie, and clearly depicts the various stages of the stacking procedure, namely, filling the injection channel (reservoir $1 \rightarrow$ reservoir 2) (snapshots 1-3); injecting the MF plug in the direction of reservoir 3 (snapshots 4-5), filling the injection channel again, and simultaneous back-flow of the injected plug into the MF crossing junction, thereby increasing the local concentration of fluorescently labeled molecules (snapshots 6-8). This procedure can be repeated a number of times, in this example by injecting the plug toward reservoir 4 (snapshots 9-12), although one could also once again inject toward reservoir 3 , until a desired local rise in concentration has been achieved; or until the MF crossing junction has been saturated with the fluorescently labeled analyte molecules (to be determined empirically). 
1

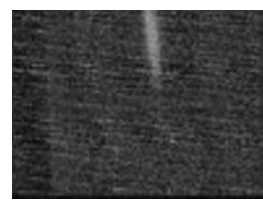

4

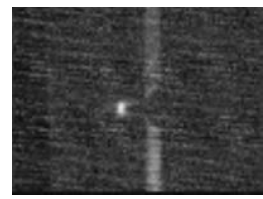

7

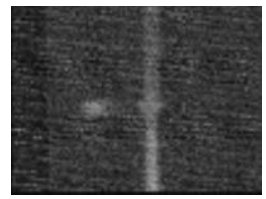

10

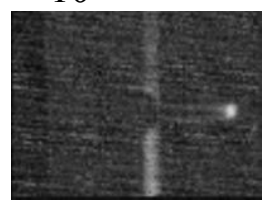

2

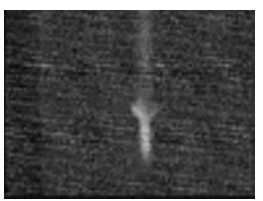

5

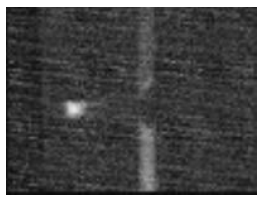

8

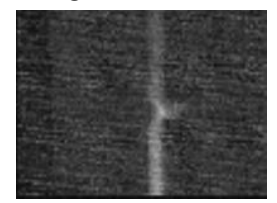

11

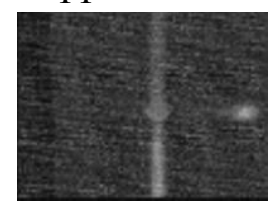

3

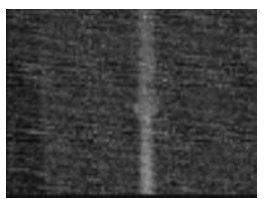

6

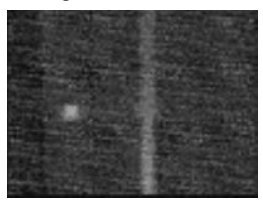

9

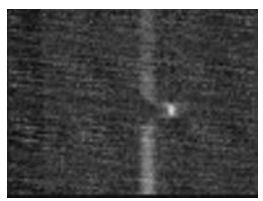

12

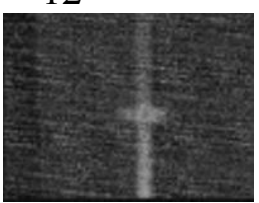

Fig. 4.4 Snapshots showing the various stages of the sample stacking procedure, consisting of filling the injection channel (reservoir $1 \rightarrow$ reservoir 2) (snapshots 1 3); injecting the MF plug in the direction of reservoir 3 (snapshots 4-5), filling the injection channel, and back-flow of the injected plug into the MF crossing junction, thereby increasing the local concentration of fluorescently labeled molecules (snapshots 6-8); repetition of this procedure in the other direction (by injecting the plug toward reservoir 4) (snapshots 9-12)

In order to quantify the advantages of the stacking procedure described in this example, we analyzed the stacking movie, whereby each frame consisted of $720 \times 576$ grayscale pixels as follows. A region of interest was selected to span over $60 \times 60$ pixels, with its center at the MF crossing junction, and the intensities of all pixels in this region were integrated at time intervals of 100 $\mathrm{ms}$ to obtain the corresponding fluorescence intensity data points, then plotted against time as shown in Fig. 4.5. 


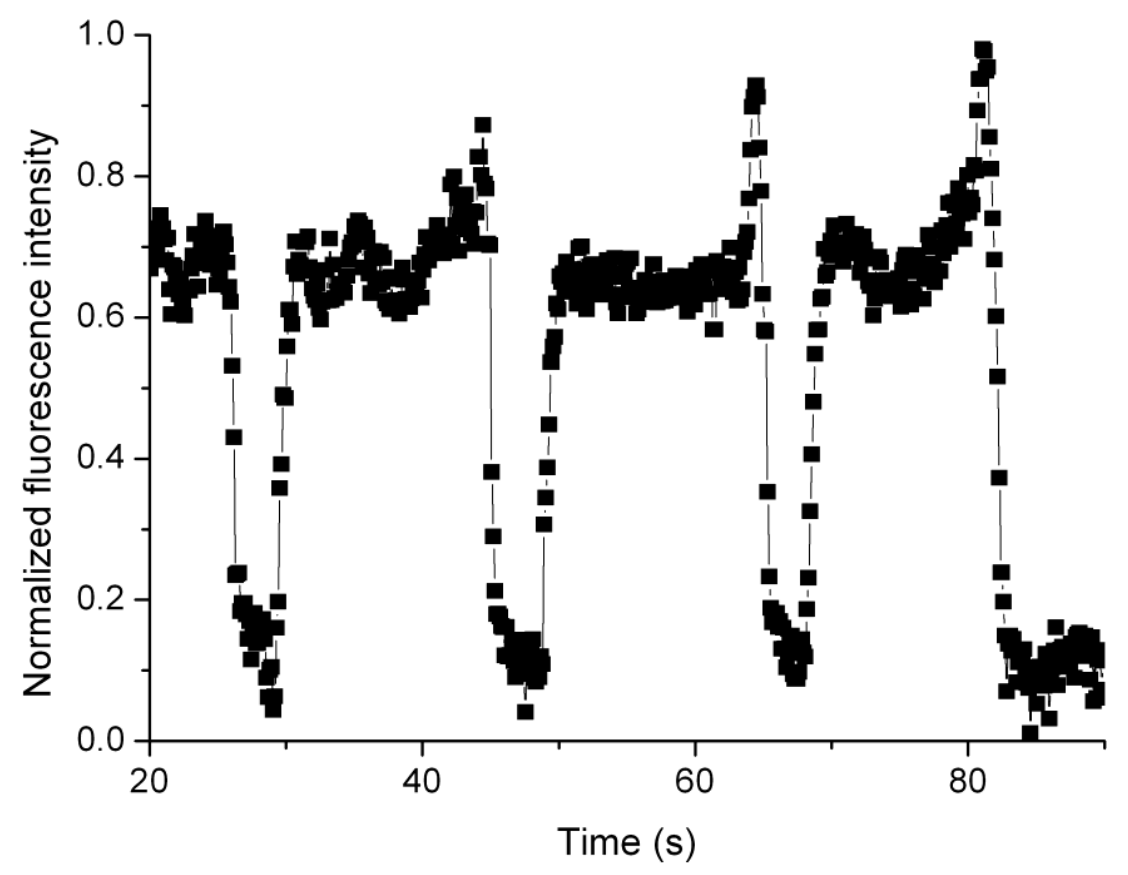

Fig. 4.5 Fluorescence intensity at the MF crossing junction plotted as a function of time during the stacking procedure; note the $\sim 35 \%$ rise in the fluorescence intensity of the MF plug as a result of running the stacking procedure three times

One can note that the fluorescence intensity of the MF analyte plug has risen by $\sim 35 \%$ as a result of the repeated stacking procedure. This procedure has been applied in the CE separation experiments involving diagnostically relevant MLPA molecules.

\subsection{All-numerical lock-in amplification}

In the previous section 4.2 we discussed a number of optical and MF techniques to increase the sensitivity of our setup. In one way or the other, all of these techniques were directly associated with the setup and its physical optimization. In this section we will apply a numerical post-processing technique to increase the setup's sensitivity by increasing the signal-to-noise ratio of the experimentally measured fluorescence signal.

If an optical beam is modulated at a specific frequency, its temporal signature leads to a similar temporal excitation of and emission by the fluorescent molecule that absorbs light at this wavelength, as long as the excited-state lifetime of the fluorescent molecule is short compared to the inverse of the modulation frequency. In other words, the fluorescence emitted by the molecule is now encoded with the same frequency as the 
excitation beam. The Fourier spectrum of the detected fluorescence signal will be composed of a peak, corresponding to the specific modulation frequency of the excitation beam. The decoding of the detected signal consists of filtering out this peak in the Fourier domain corresponding to the single modulation frequency, to extract only the signal corresponding to the specific excitation wavelength. By applying inverse Fourier transforms, the corresponding individual time-domain signal is regenerated, minus the background, broadband noise. This will result in an enhancement of the signal-to-noise ratio, and thereby the setup's sensitivity. We implement this entire procedure numerically by means of a post-processing program that can be applied to any measured, especially non-modulated fluorescence signal. We therefore present a numerical analogue of the so-called "phase-sensitive detection" or "lock-in amplification [Horlick, 1975] [Blair, 1975] [Temple, 1975] [Wolfson, 1991], a technique that has found its way also in numerous biochemical detection strategies, including those involving sensitive fluorescence detection [Novak, 2007] [Rivera, 2008]. A frequency domain description of the lock-in amplification technique has been described in the literature earlier [Scofield, 1994] and applied in a variety of domains, e.g., synchronic audio frequency detection [Gonzalez, 2007], liquid crystal density measurements [Poterasu, 2008], measurements on the fluctuations in laser frequency [Siemsen, 2005], high-speed imaging [Uchida, 1996], etc. We have applied this well known technique in the context of fluorescence monitoring during CE separations to enable the detection of the electropherogram peaks buried under the background noise. This procedure consists of the following steps.

We multiply the measured signal with a square-wave function, let's call it SQ1 (to simulate the real life situation of a mechanical chopper cutting off and turning on the laser beam at regular time intervals during the measurement), (with 1 as the maximum value, and 0 as the minimum) having a period equal to a multiple of the sampling period of the signal. The resulting signal is modulated a posteriori but it is as if it were modulated $a$ priori. It will now be processed in the Fourier domain. Discrete Fourier transform of the modulated signal leads to its spectrum which is essentially a frequency-shifted (due to the modulation) version of the original spectrum of the signal. We now filter out the single peak around the "modulation frequency" and perform an inverse Fourier transform on the filtered signal to return the time-domain signal with a lower noise. Furthermore, we also multiply the original signal by a square wave SQ2, which is simply a temporally shifted, out-of-phase version of the square wave SQ1, and apply the same processing steps as described above. As the final step we add the two signals obtained by such post-processing with the two out-of-phase square waves. By using simply one square wave we obtain a 7-fold SNR 
enhancement. The addition of the result from processing with the second (phase shifted) square wave delivers another factor of $\sqrt{2}$, thereby increasing the total enhancement to up to 10-fold, i.e. almost by an order of magnitude.

Next, we will, as an illustration, apply the procedure described above on a computer generated Gaussian signal with an added random noise (Fig. 4.6), and then on the measured electropherogram of Fig. 4.3(bottom).

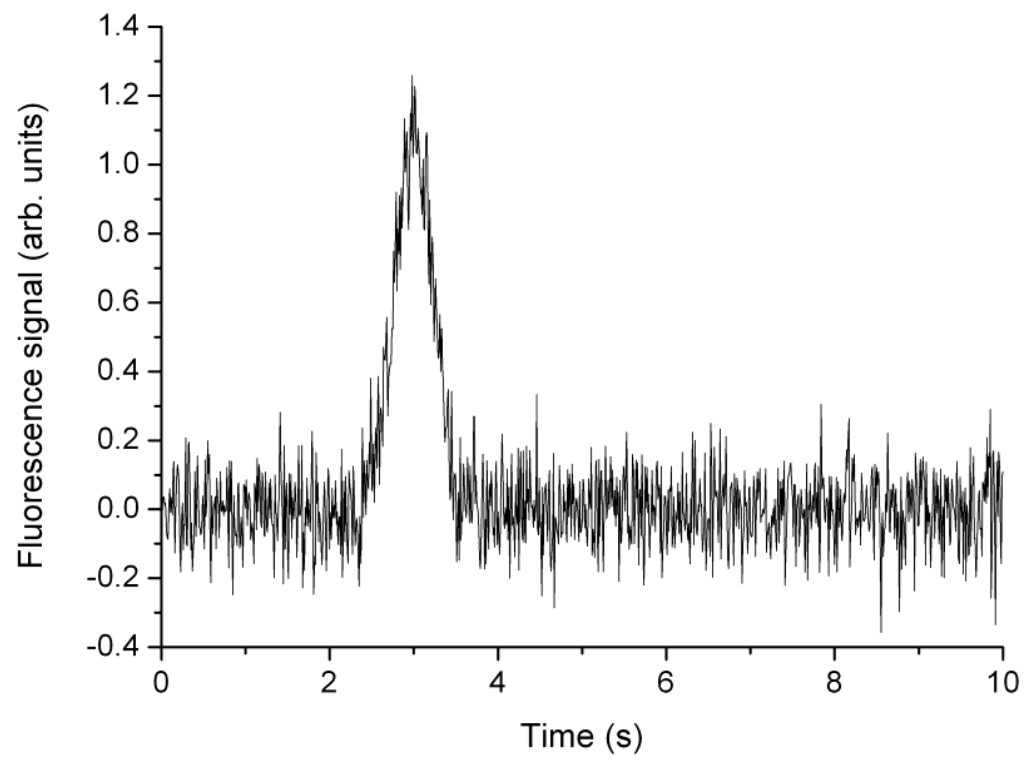

Fig. 4.6 A computer generated Gaussian signal with added random noise

The standard deviation of the noise around its average in Fig. 4.6 is 0.095 , corresponding to an SNR of 12 . We multiply this signal by a square wave, in this case having a period of $60 \mathrm{~ms}$ or, in other words, a frequency of 16.67 Hz. The spectrum of the resulting "modulated" signal is shown in Fig. 4.7 . 


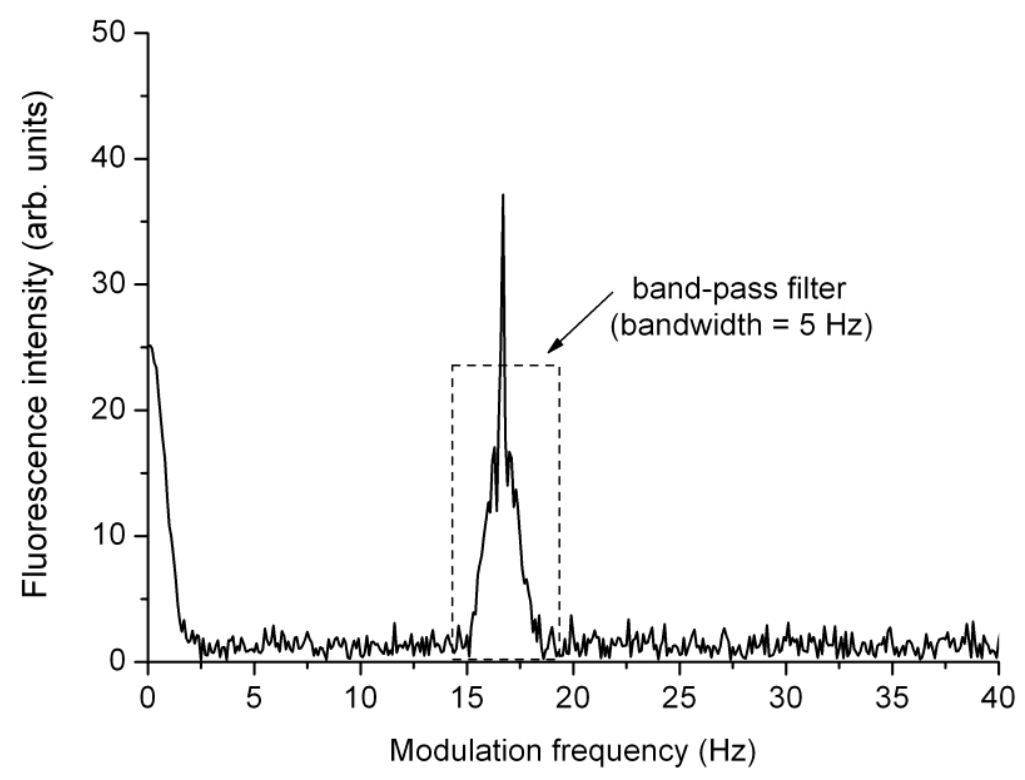

Fig. 4.7 A part of the Fourier spectrum of the fluorescence signal after the a posteriori modulation by multiplication with a square wave; and the dotted line showing the rectangular band-pass filter function

The spectrum of the signal around the "modulation" frequency of 16.67 $\mathrm{Hz}$ is extracted by means of a rectangular band-pass filter (bandwidth $=5$ $\mathrm{Hz}$ ) centered at $16.67 \mathrm{~Hz}$. The time-domain signal resulting from an inverse Fourier transform is shown in Fig. 4.8. The standard deviation of the noise around its average in the processed signal is $\sim 0.0149$, corresponding to an SNR of 74. In comparison to the signal in Fig. 4.6, we see an up to 7-fold improvement in the SNR. A further improvement by a factor of $\sqrt{2}$ is obtained by means of yet another processing step with an out-of-phase modulating square wave, as described earlier, leading to a total enhancement of up to 10 -fold. 


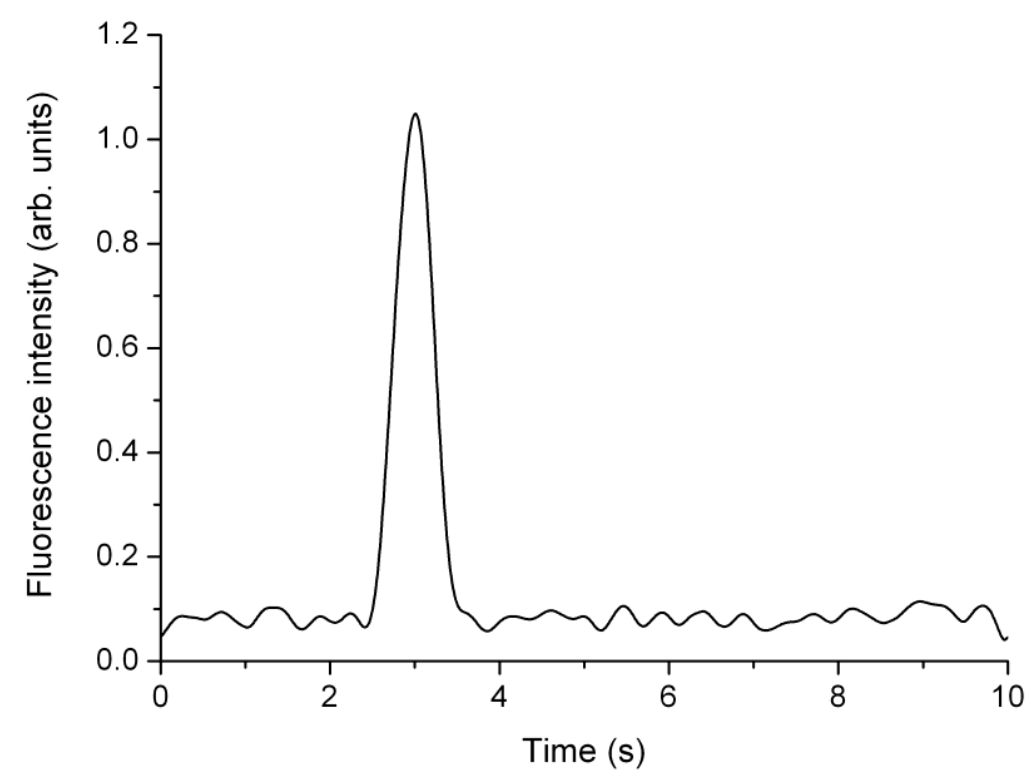

Fig. 4.8 Fluorescence signal generated after the all-numerical lock-in amplification procedure

We also apply this technique to two measured electropherograms (Figs. 4.9 and 4.10) for a 17-molecule dsDNA ladder that has already been studied for its MF performance in chapter 3. In these examples, up to 10-fold SNR enhancement was realized without inducing significant broadening of the (typically the sharpest) peaks. This corresponds to a final LOD of $220 \mathrm{fM}$. This corresponds to the detection of 8-9 dsDNA molecules in the excitation volume. One previous approach [Yin, 2006] [Yin, 2007] utilizing liquid-core WGs for optofluidic applications reports the detection of single liposome molecules. The liposomes used in this work however were typically sized $200 \mathrm{~nm}$, and each liposome was intercalated with $\sim 400$ dye molecules. The average size of our dsDNA molecules is $\sim 150 \mathrm{~nm}$ when completely stretched. They are typically folded or coiled, making them even smaller. We also have fewer dye molecules per dsDNA molecule, even in our largest dsDNA molecule (3000 bp), we have only 300 dye molecules with an intercalation period of 1 dye / $10 \mathrm{bp}$. Our achieved LOD is therefore significant in the field of integrated optofluidics. 

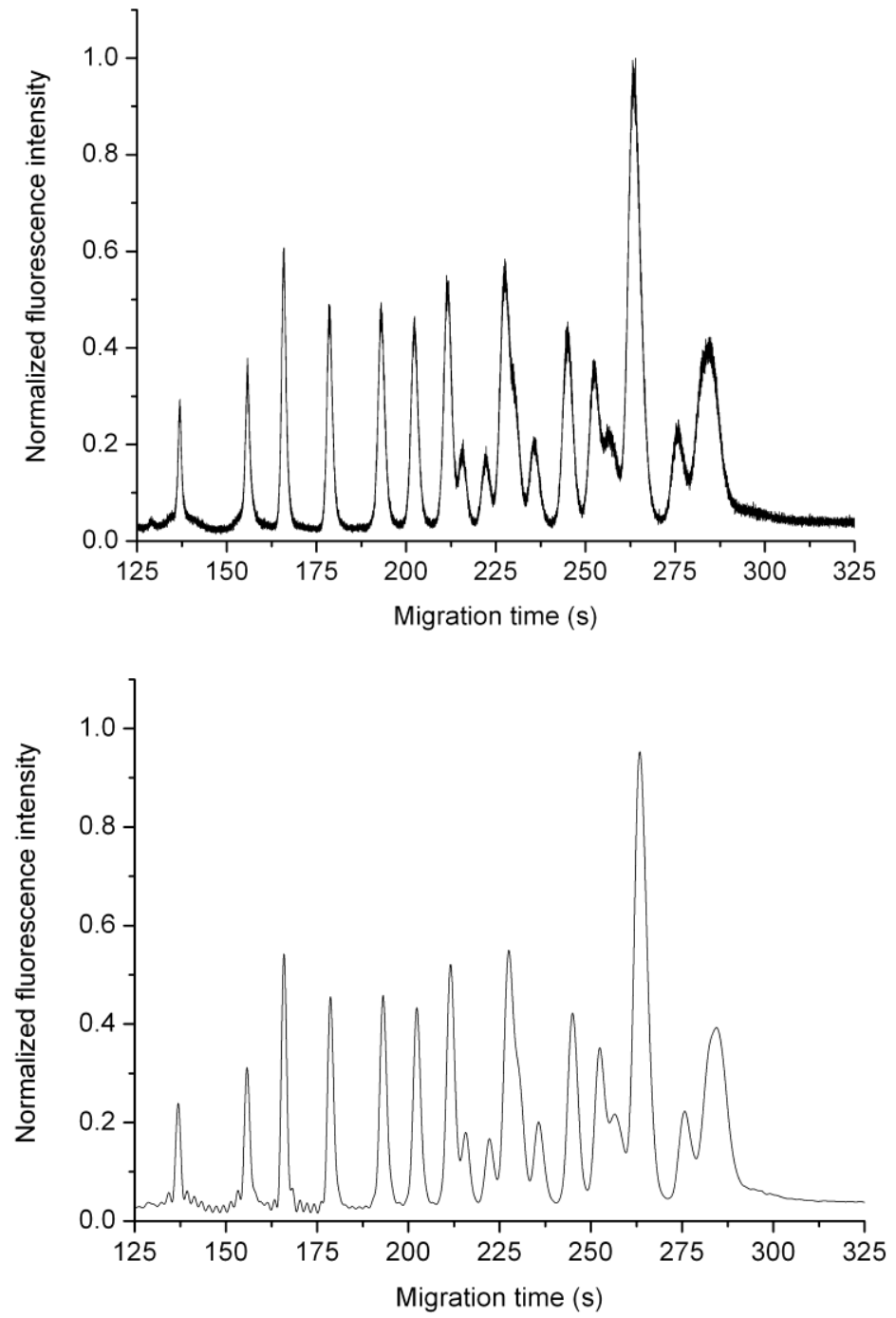

Fig. 4.9 Fluorescence signal generated after applying the all-numerical lock-in amplification procedure to the measured electropherograms for a 17-molecule dsDNA ladder 

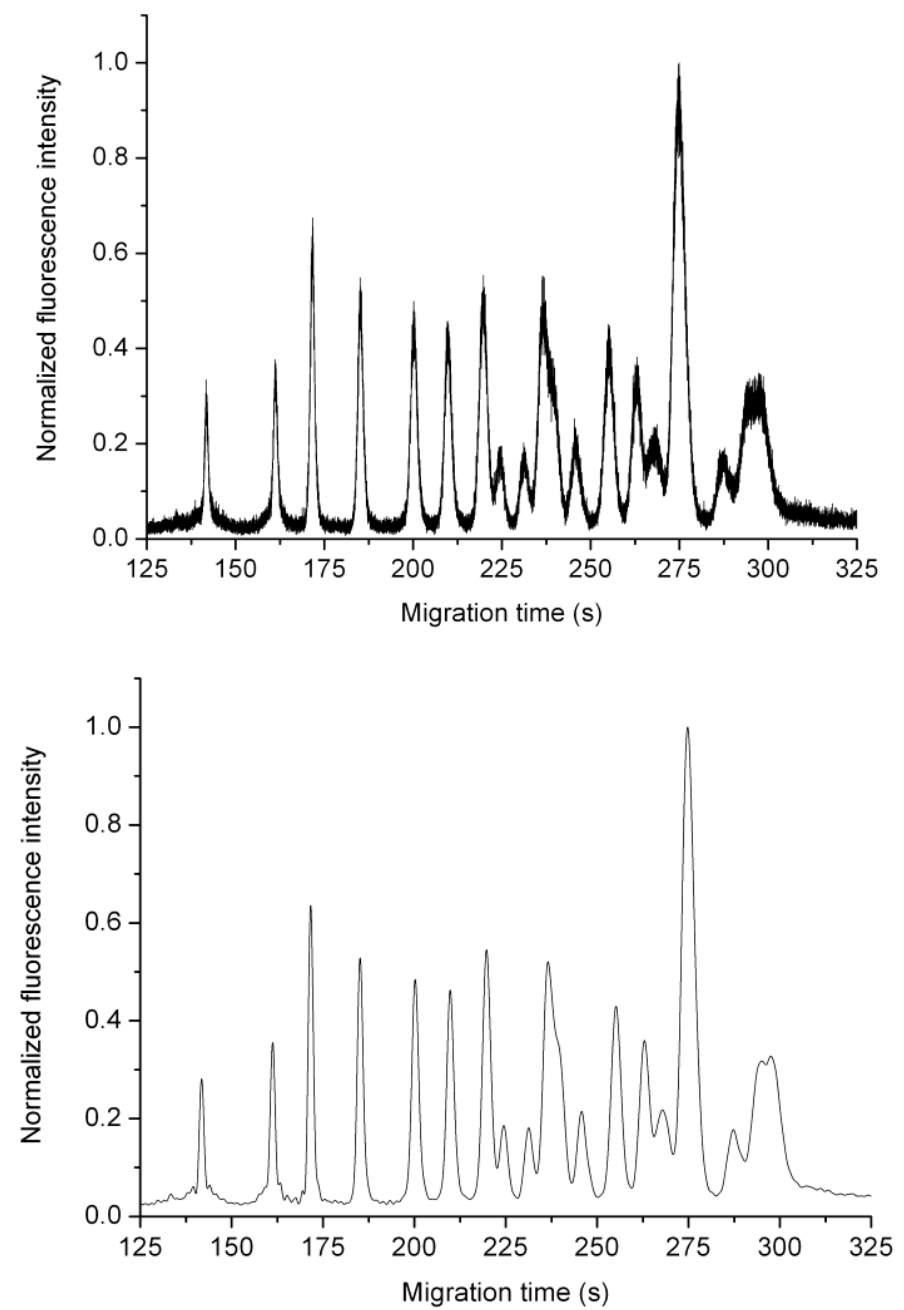

Fig. 4.10 Fluorescence signal generated after applying the all-numerical lock-in amplification procedure to the measured electropherograms for a 17-molecule dsDNA ladder

This example demonstrates the capability of this approach to visualize fluorescence signals obtained during CE separation that would otherwise have been lost in the background noise. This further paves the way toward the high sensitivities that we require for the detection of covalently fluorescently end-labeled, diagnostically relevant dsDNA molecules during their CE separation and analysis, as will be described in the following chapter 5 . As this technique does not imply any modification whatsoever in an existing fluorescence detection setup, but only the use of a PC for Fourier analysis on an already measured fluorescence data, it is an attractive and cost-effective approach to achieve SNR enhancement. Besides, it avoids the use of bulky equipment such as a physical lock-in amplifier, or a mechanical 
chopper to modulate the excitation laser beam. This is an added advantage for the miniaturization of fluorescence detection and portability of a subsequent commercial optofluidic LOC device based on this measurement principle.

\subsection{Summary}

In this chapter we described a number of different approaches that were adopted in order to reach a highly sensitive fluorescence detection setup that will be used for dsDNA analysis experiments based on CE separation in an optofluidic chip, to be described in detail in chapter 5, section 5.3. We started with simple, but nevertheless practically useful steps, such as suppressing the background fluorescence by painting the MF chip and the MF cartridge with black ink, and by the use of suitable wavelength filters. Use of integrated optical waveguides for laser excitation, with a wellconfined excitation spot instead of a broadband, non-coherent Hg-arc lamp lead to a further order-of-magnitude increase in the sensitivity. Furthermore, a MF protocol implemented by J. van Weerd from Zebra Bioscience BV for stacking the fluorescent analyte sample at the MF crossing junction, to increase its effective concentration during injection resulted in an additional $\sim 35 \%$ effective rise in the concentration of the injected plug, i.e. w. r. t. the total number of analyte molecules introduced to the MF reservoir at the start of the experimental run. Finally, we achieved up to 10-fold enhancement of the sensitivity by implementing lock-in amplification as an all-numerical post-processing approach. This result paved the way for ultrasensitive fluorescence monitoring of diagnostically relevant dsDNA analysis based on CE separation. The all-numerical approach implied the use of merely a PC for the analysis, thereby eliminating the need for bulky detection apparatus such as choppers, lock-in amplifiers, etc. Nevertheless, the use of choppers or other modulation instrumentation is critical for multi-wavelength analyses, as it not only enables as high sensitivity as the all-numerical approach described here, but also multi-color diagnostics by exclusive labeling of dsDNA molecules as per their origin, followed by unambiguous, simultaneous detection. This aspect will be further elaborated in section 5.3 of chapter 5 . 


\section{Multi-color fluorescent DNA analysis}

\subsection{Introduction}

In chapters 3 and 4, we discussed the optimization of the MF parameters, as well as a method to improve the signal-to-noise ratio during fluorescence sensing, which enabled us to perform the electrophoretic separation and quantitative analysis of double-stranded DNA (dsDNA) molecules in the diagnostically relevant size range, at a low LOD of $220 \mathrm{fM}$, when working with intercalating fluorescent dye labels. This improved functionality sets the stage for a fundamentally distinct analysis scheme, as we will describe in this chapter, potentially leading to a paradigm shift in dsDNA analysis.

Commercial systems [Bio-rad, web] as well as laboratory setups [Kuswandi, 2007] for the LIF monitoring [Johnson, 2004] of electrophoretic DNA separation consist of a single excitation wavelength in order to induce fluorescence in the labeled analytes. In other fields such as flow cytometry, many commercial instruments do utilize multi-wavelength laser excitation. Some commercial instruments, e.g. the Roche LightCycler 480 [Iten, 2007] [Roche, web], approach multi-wavelength fluorescence sensing for bimolecule separation and analysis, by means of a broadband light source such as a Xe lamp, extracting the multiple wavelengths with monochromators. These instruments tend to apply a number of complicated optical schemes with bulky equipment in order to achieve an unambiguous separation of signals at different fluorescence wavelengths, which is critical to any multi-wavelength approach. Also in the scientific literature, there have been reports of multi-wavelength LIF detection strategies, based on, e.g., an integrated spectrometer on a chip [Schmidt, 2007], or an external wavelength selective grating [Goetz, 2007] to separate the concerned wavelengths by use of a color CCD array [Karger, 1991], by separately collecting the differently colored fluorescence signals using fibers and detecting them with multiple avalanche photodiodes [Zhu, 2004], or by use of an external spectrum analyzer [Sugino, 2009] [Lin, 2008].

In section 5.2 we describe the implementation of a dual-point fluorescence excitation / detection scheme, with single or dual wavelength excitation and a single detector for the multiple detection points The potential of this approach will be briefly described, as a precursor to the following sections 5.3 and 5.4 in which we describe a generic and universally applicable method for multi-color analysis. In the approach described in sections 5.3 and 5.4, we launch simultaneously two laser beams at different wavelengths through a single $\mathrm{WG}$, encode the fluorescence emitted by each set of end-labeled DNA molecules by exclusively time- 
modulating the corresponding excitation laser beam, and subsequently decode the emitted and detected fluorescence by Fourier analysis. In this manner we separate differently encoded fluorescence signals from each other, thus enabling simultaneous multi-color interrogation of multiple sets of dsDNA fragments of different origin in a single small detection volume with a single ultrasensitive, albeit color-blind photomultiplier tube (PMT). We believe that this approach is more elegant in comparison to the approaches explored earlier that depend upon additional, expensive, external hardware in order to achieve the wavelength separation, resulting invariably in a bulky setup around the tiny microchip.

Finally, in section 5.5 we will summarize the presented multi-color fluorescent analysis with modulation-frequency encoded multi-wavelength excitation, as a technique which may be beneficial to a wide range of applications encountered within scientific research.

\subsection{Dual-point fluorescence sensing}

In this section we will describe the on-chip implementation of dual-point fluorescence sensing, with possible duplexing by means of dual-wavelength excitation, using up to four optical WGs, all crossing the MF CE separation channel at different points.

By measuring at two different points in a MF channel simultaneously, using two detection windows (DWs), it is possible to deduce information about the variations in flow speed (if any) and plug broadening (if any) during migration between these two points by observing passage durations and peak heights. If there is indeed plug broadening, the passage durations will be longer as one follows the plug along the MF channel, and the peak heights will continuously decrease, because the effective concentration of the plug contents will reduce owing to diffusion. If a number of species with electrophoretic mobilities close to each other are to be separated, it is expected that some of them with a relatively larger mobility difference will separate after shorter migration distance, whereas the others that have mobilities very similar to each other will separate only towards the end of the separation channel. By measuring at a point on the chip further away from the separation point, it will be possible to observe the corresponding response peaks separately. Alternatively, species that are present at low concentrations and well separated from the rest, will give rise to a weak fluorescence peak at the first DW and might completely "disappear" (by being obscured owing to background noise) when migrating further. So, by use of dual point excitation extra information may be attained which otherwise would have been lost. Such and other possible advantages of multi-point fluorescence excitation and detection during CE separation have 
long been researched in the case of bulk capillaries [Hjerten, 1992] [Sun, 1995] [Srichaiyo, 2001] and our work supplements these works with suitable examples on a microchip.

In some cases it is advantageous to apply, in addition to dual-point excitation, also dual-wavelength excitation (see Fig. 5.1), as demonstrated in the literature [Stefanowicz-Hajduk, 2009] by making use of commercial CELIF instruments specifically modified for dual-wavelength excitation / detection [Beckman Coulter 1, web]. Such an approach could very well be used in a diagnostic application, where one wishes to compare an unknown sample, e.g. from a gene region under investigation for mutations, with a reference sample, e.g. from the same gene region corresponding to a healthy person. One would expect in this case that the base-pair (bp) differences between the corresponding pairs of analyte dsDNA molecules would be small (1-2 bp), if any. These differences could be determined by exclusively labeling the reference sample and the sample under investigation and then flowing them against each other and monitoring their separation with dualpoint, dual-wavelength fluorescence excitation / detection. One of the advantages of using two well separated waveguide pairs, corresponding to the two DWs, is that peaks of the two differently labeled molecular species that coincide in the signals of one of the DWs, in general do not coincide with the signals coming from the other DW.

In this section, we will illustrate dual-point, dual-wavelength monitoring with single-stranded DNA (ssDNA) separation, in order to demonstrate simultaneous dual-wavelength excitation without cross-talk.
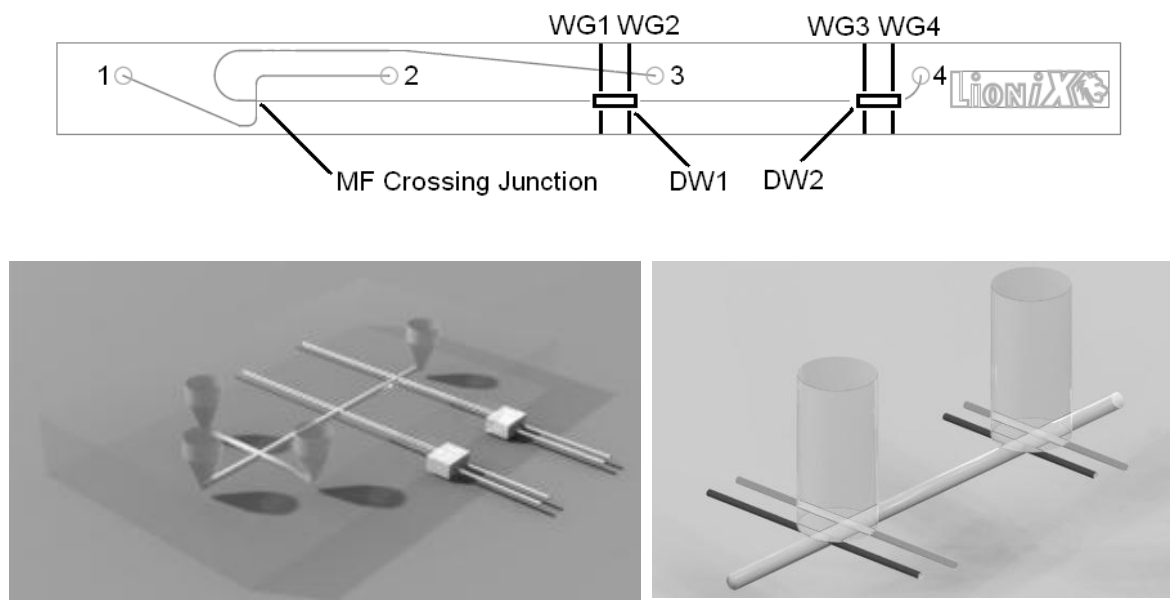

Fig. 5.1 (top) Layout and (bottom-left) 3D schematic of an optofluidic chip indicating the MF reservoirs, MF channels, and the two DWs comprising of two WGs each, each WG crossing the MF CE separation channel perpendicularly in plane; (bottom-right) close-up of a MF channel with the two DWs indicated. 


\subsubsection{Materials and methods}

Figure 5.1 shows the layout of an optofluidic chip with two detection windows, each consisting of the interface region of two WGs carrying two different wavelengths intersecting the MF CE separation channel in plane at four different locations, see Fig. 5.1, at distances of $2.0 \mathrm{~cm}$ (WG1), $2.1 \mathrm{~cm}$ (WG2), $3.5 \mathrm{~cm}$ (WG3), and $3.6 \mathrm{~cm}$ (WG4), from the MF crossing junction at which the separation commences. In the next subsection we will describe three experiments in which each time two differently covalently end-labeled ssDNA molecules (as opposed to the intercalating dye labeled dsDNA molecules used in the previous chapter) are flown through the MF channel. As end labels the fluorescent dyes Alexa Fluor 647 (AF647) and Cyanine 3 (Cy3) are used, and the sizes of the molecules are $(15,20),(19,20)$, and $(19,19)$ nucleotides (nt) for the three experiments. Hereby the first number for the nts corresponds to AF647, the second to Cy3 labeling.

The inner walls of the entire MF channel network were pre-coated with an EPDMA-based polymer [Cretich, 2003], and filled with a gel matrix consisting of hydroxypropyl-cellulose [Sanders, 2003], dissolved (3\% wt./vol.) in $20 \mathrm{mM} \mathrm{MES} \mathrm{/} 20 \mathrm{mM}$ His buffer ( $\mathrm{pH}$ 6.2). Red He-Ne $(\lambda=633$ $\mathrm{nm})$ laser beams were coupled into WG1 and WG3, while green $\mathrm{He}-\mathrm{Ne}(\lambda=$ $543 \mathrm{~nm}$ ) laser beams were coupled into WG2 and WG4. A cooled photomultiplier tube (PMT) (H7421-40, Hamamatsu Photonics K.K.) was built onto the output port of an inverted microscope (DMI5000M, Leica Microsystems $\mathrm{GmbH}$ ) and optimally aligned to collect light from the two detection windows, DW1 and DW2, which are located in the regions where WG1/WG2 and WG3/WG4, respectively, intersect the MF CE separation channel. An appropriate multi-band filter (XF57, Omega Optical, Inc.) ensured that only the fluorescence signals emitted by the analyte ssDNA molecules reached the PMT.

The flow protocol was based on driving voltages of up to $1.2 \mathrm{kV}$, delivered by Pt electrodes integrated into the MF reservoirs, with the help of a LabVIEW script to steer the microfluidic control system (Capella, CapiliX $\mathrm{BV}$ ). The volume of a typical ssDNA mixture plug upon injection into the MF CE separation channel was approximately 605 picoliters.

\subsubsection{Experimental results and discussion}

The first of the three experiments (corresponding to 15 and $20 \mathrm{nt}$ ) was performed as follows. Initially, only a single species (15-nt-ssDNA-AF647) was CE-flown through the MF separation channel. This CE flow experiment was repeated twice, firstly with only the red He-Ne laser turned on, in which case a strong signal peak was observed from DW1 and DW2 when the 
fluorescently labeled ssDNA plug crossed WG1 and WG3, respectively, and secondly with only the green He-Ne laser turned on, in which case the signal measured from both DW1 and DW2 matched with the baseline detector signal. This procedure was then repeated for the other species (20-nt-ssDNACy3), leading to the opposite result. Thus, it was experimentally confirmed that cross-excitation between the two species was, for all practical purposes, absent (see Fig. 5.2, top).

The two species were then mixed and the resulting polychromatic ssDNA mixture was separated by $\mathrm{CE}$ into the individual monochromatic ssDNA components in the MF CE separation channel. Figure 5.2 (bottom) depicts the corresponding electropherogram based on the fluorescence signal measured from both DWs. Since cross-excitation is absent, the two peaks (separated by $\sim 4 \mathrm{~s}$ ) after a migration time of around $20 \mathrm{~s}$ correspond to the two separated species as detected via DW1, each being excited by the corresponding laser wavelength of $633 \mathrm{~nm}$ (from WG1) or $543 \mathrm{~nm}$ (from WG2), respectively. Similarly, the response after 70-80 s corresponds to the fluorescence signal measured from DW2, where the separation between the fluorescence peaks of the two plugs now amounts to a larger delay of approximately $11 \mathrm{~s}$ because of the larger distance of DW2 from the starting point, namely the MF crossing junction.

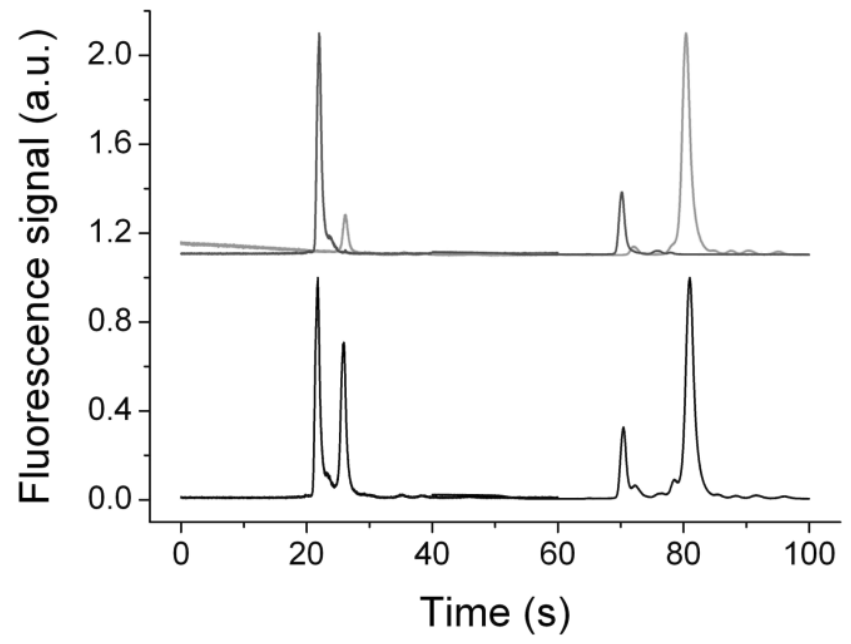

Fig. 5.2 Electropherograms depicting the CE separation of two ssDNA molecules, as seen from the two DWs; top, red and green colored curves, individual signals as measured with a single wavelength and temporally superimposed on each other; bottom, cumulative signal measured by the PMT during simultaneous dualwavelength monitoring 
Comparison of the top and bottom electropherograms in Fig. 5.2 yields identical temporal positions of the fluorescent peaks, hence proving that successful dual-point, dual-wavelength fluorescence monitoring of the MF CE separation of ssDNA molecules has been achieved.

An essential advantage of the application of the dual-point, dualwavelength approach is that the intrinsic, fluidic CE separation resolution may be further enhanced by the inherent spatial separation introduced by the WG array. As a result, even molecules with similar or exactly the same size, but with a different colored fluorescent label, which naturally flow at the same speed, can nevertheless be detected as individual electropherogram peaks as they cross the appropriate waveguide carrying the corresponding unique excitation wavelength for the label. This performance is exemplified in Figs. 5.3 and 5.4 - electropherograms obtained exclusively from DW2 where ssDNA molecules with a single-nucleotide size difference and with no size difference, respectively, are mutually optically "separated".
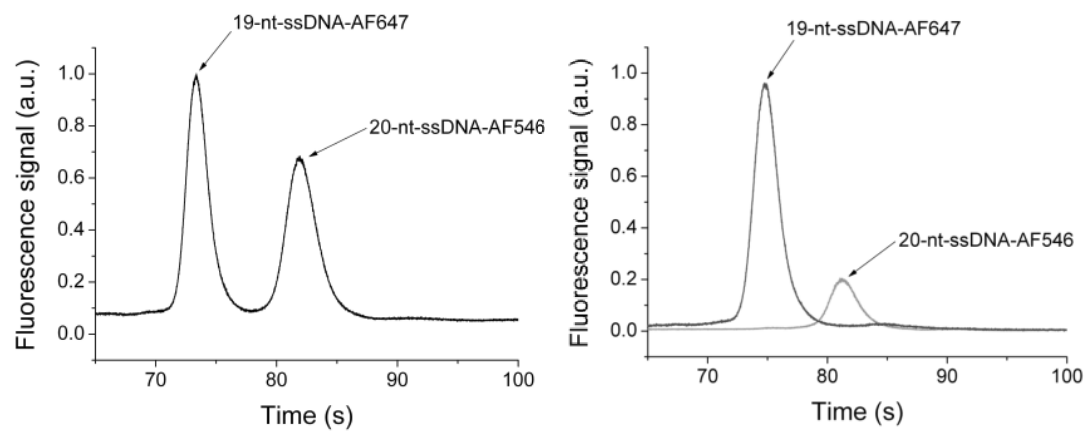

Fig. 5.3 Electropherograms based on the fluorescence signal generated by dualpoint, dual-wavelength excitation (left), and generated by dual-point with two times single-wavelength excitation and temporally superimposed on one another, as measured from DW2 (right), for ssDNA molecules with a size difference of a single nucleotide (19 and $20 \mathrm{nts}$ )
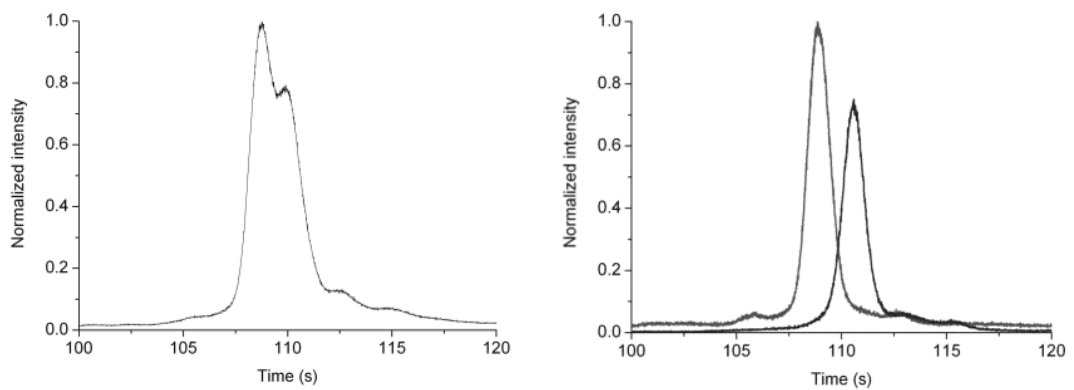

Fig. 5.4 Electropherograms based on the fluorescence signal generated by dual-point, dual-wavelength excitation (left), and generated by dual-point with two times single-wavelength excitation and temporally superimposed on one another, as measured from DW2 (right), for ssDNA molecules with exactly the same size (both 19 nts) 
In conclusion to this section on this dual-point approach with single or dual excitation we note that the method will have an added value in a number of situations, depending on the molecular species to be investigated. However, the method will be cumbersome in case of complicated electropherograms or in the case, for example, of excitation of a larger number of different labels with a corresponding number of different wavelengths, in which case the resulting peaks may become inseparable. A more universal method for which the latter does not apply is introduced and discussed next.

\subsection{Modulation-frequency encoding and Fourier- analytical decoding}

In order to exploit the true potential of multiplexing for real life applications, we will need a generic technique that could universally work for a large number of excitation wavelengths being used simultaneously, to excite as many different fluorescent labels attached to molecules to be analyzed via $\mathrm{CE}$. In this and the following section we present an elegant approach to achieve such multi-color analysis, by means of a priori encoding of the fluorescence originating in different biomolecules by intensity-modulating the light sources, followed by Fourier decoding of the detected biophotonic signals, thereby tracing back the origins of the biomolecules.

\subsubsection{Description of the principle}

If an optical beam is modulated at a specific frequency, its temporal signature leads to a similar temporal excitation of, and emission by, the fluorescent molecule that absorbs light at this wavelength, as long as the excited-state lifetime of the fluorescent molecule is short compared to the inverse of the modulation frequency. In other words, the fluorescence emitted by the molecule is encoded with the same frequency as the exciting beam. In the case of simultaneous presence of multiple analytes with distinct absorption bands, each analyte can be excited by a corresponding optical beam with a distinct modulation frequency, as shown in the schematic of Fig. 5.5. Needless to say, this generic method can be applied also to other optical measurement principles, e.g. absorption and interferometry. If the different modulation frequencies are harmonically uncorrelated with one another, all the resulting fluorescence signals can be unambiguously attributed to the presence of the corresponding analyte molecule, even if all these fluorescence signals happen to occur at the same wavelength or if the fluorescence occurs at different wavelengths but a wavelength-insensitive 
detection system, e.g. an ultrasensitive PMT without monochromator, is used. As a result, the Fourier spectrum of the detected cumulative fluorescence will be composed of individual, non-overlapping peaks, each corresponding to the specific modulation frequency of its exciting beam.
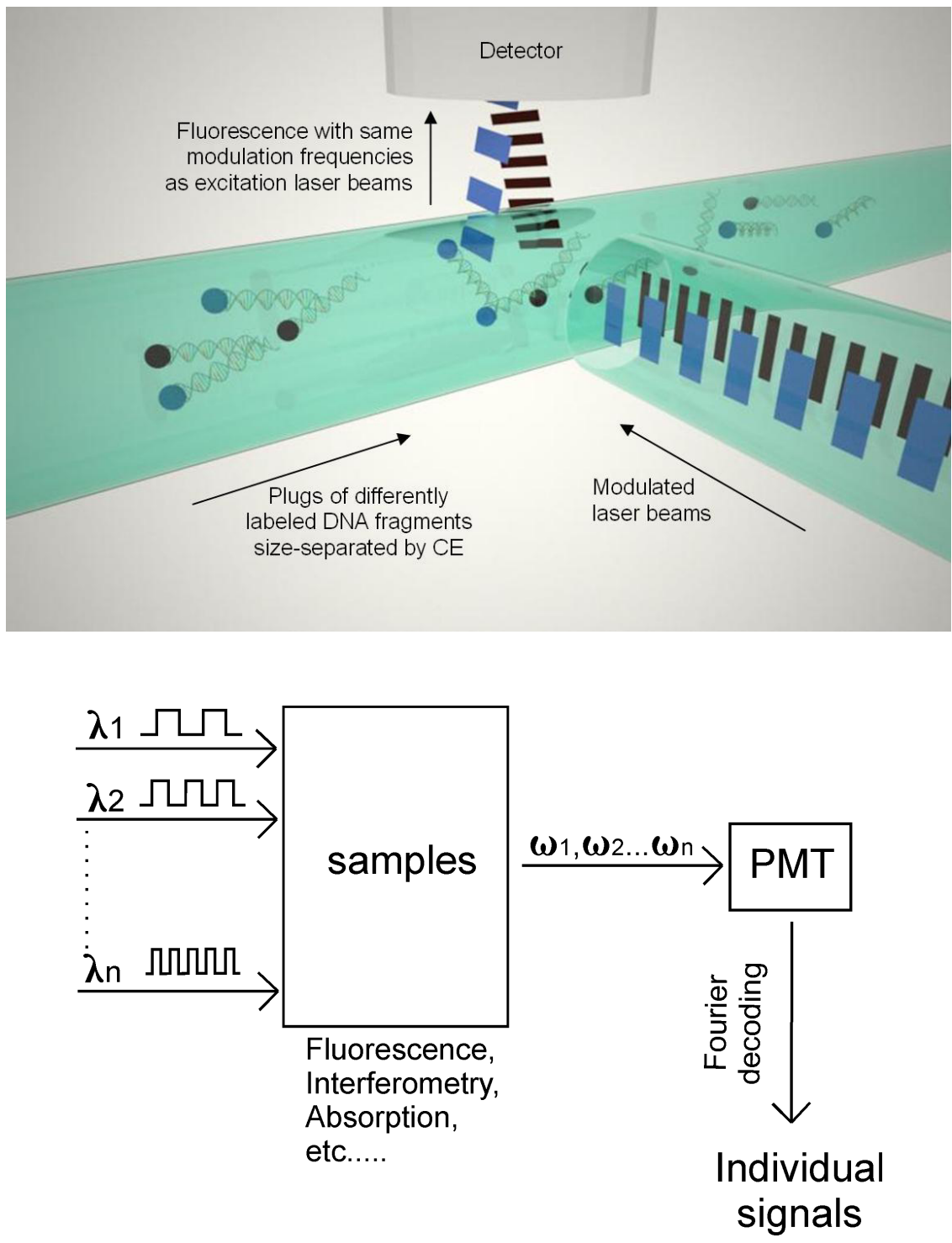

Fig. 5.5 (top) Schematic showing plugs of exclusively fluorescence-labeled molecules migrating through the MF separation channel, intersecting the excitation $W G$ which guides laser light of different wavelengths and modulation frequencies, and a plug containing DNA molecules with two different labels emitting fluorescence light at the two modulation frequencies while crossing the excitation $W G$; (bottom) a block-diagram depicting the generic principle of the method 
The decoding of the detected signal therefore consists of filtering out the peaks in the Fourier domain corresponding to the different modulation frequencies, to unambiguously extract the signal corresponding to a specific excitation wavelength. By applying inverse Fourier transform, the corresponding individual time-domain signals are regenerated. It must be noted though that such numerical analysis will typically involve a discrete Fourier transform, resulting in a well-defined, bounded range of frequencies (defined for example by the number of temporal data points). All the higher harmonics and all the possible linear combinations thereof will essentially fall in this range, or will be folded back around the right-hand extremity of the range, to nevertheless appear to be in the range. Such aliasing effects must also be taken into account, while choosing the correct frequency peaks in the Fourier domain for applying the inverse Fourier transform.

\subsubsection{Experimental proof of principle}

As a proof of principle, we monitored the migration of sets of differently sized biomolecules through an excitation/detection area. Here two sets of fluorescently-labeled biomolecules were used, each set consisting of two differently sized ssDNA molecules, with sizes of 19 and 23 nucleotides, respectively, which migrate with different speeds. One set was red-labeled, the other blue-labeled. Two laser beams at the absorption wavelengths of the labels, intensity-modulated at frequencies $f_{1}$ and $f_{2}$, respectively, were launched into the excitation/detection area through two adjacent WGs separated by $500 \mu \mathrm{m}$. For fluorescence detection, a PMT (H7421-40, Hamamatsu Photonics K.K.) cooled down to 203 K was aligned to collect the fluorescence signal from the excitation/detection area. The modulation of each laser induced an accordingly time-varying fluorescence signal emitted by those dye molecules absorbing this specific laser wavelength, and the resulting time-modulated fluorescence signal at both wavelengths was simultaneously recorded by the ultrasensitive, albeit color-blind PMT, as shown in the upper parts of Fig. 5.6. Fourier analysis was implemented in Matlab (MathWorks, Inc.) for back-end processing of the recorded total fluorescence signal, resulting in a frequency spectrum which consists of signals at the different modulation frequencies. Only a narrow band of frequencies $(\Delta f=2 \mathrm{~Hz})$ around each modulation frequency $\left(f_{1}=17 \mathrm{~Hz}, f_{2}=\right.$ $31 \mathrm{~Hz}$ ) was selected by a flat-top transfer function and extracted back into the time domain by an inverse Fourier transform, leading to the blue and red colored graphs in the lower parts of the images in Fig. 5.6. The intensities at the higher harmonics of the fundamental frequencies were neglected during the inverse Fourier transform, as this procedure ensured the best signal-to- 
noise ratio. Note that the selected band of $\Delta f=2 \mathrm{~Hz}$ is wide enough to cover the frequencies corresponding to the different response peaks, that in the time domain have a typical width of 2-3 s. On the other hand, this selected bandwidth is small enough to reject most of the noise.

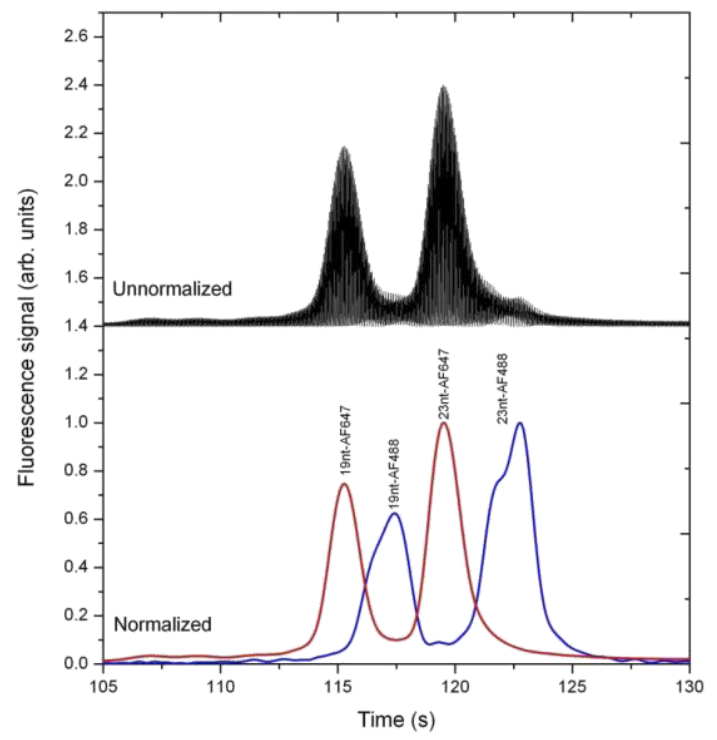

Fig. 5.6 Fluorescence intensity vs. time as measured by a color-blind PMT during the migration of two sets of blue-and red-labeled biomolecules through the excitation/detection area under modulated excitation: cumulative signal from all four molecules (upper part) and corresponding individual signals resolved by Fourier decoding (lower part)

From these examples we learn that dual-wavelength fluorescence monitoring of CE separation is feasible without cross-excitation, and equally importantly, that no physically unrealistic features are introduced to the timedomain signal as a result of the Fourier decoding. This easily applicable optical detection scheme enables exciting possibilities in many fields, whenever multiplexing can add value to a measurement system, e.g., when it provides an advantage or is even imperative to investigate different sets of biomolecules in a single sample volume and to identify their origin. The examples described show that the simultaneous investigation of several independent and exclusively labeled biomolecule sets is feasible in a single experiment. In this thesis, we have focused on a specific example of this case, where the different biomolecules originate from diagnostically relevant regions of different genes and are analyzed in a single experiment, based on the well-chosen wavelength labeling of the different genetic origins and encoding of their fluorescence by different modulation frequencies. In the following subsection we will describe such a diagnostic application. 


\subsection{Application of modulation-frequency encoding to multiplex genetic diagnostics}

While in the dsDNA analysis of chapter 3 we employed intercalating dye molecules, which bind to dsDNA molecules in multiple copies, thereby producing a large fluorescence signal, thanks to the improved sensitivity (as described in chapter 4) we are now able to detect even end-labeled dsDNA molecules producing - owing to the decrease in the total number of fluorescence emitters per analyte molecule - a far lower fluorescence intensity. Contrary to intercalating dyes which unspecifically bind and unbind at short time intervals to any dsDNA molecules in their vicinity [Lei, 1969] [Zipper, 2004] [Olson, 2004] [Biver, 2005], end-labels are attached covalently and permanently during a PCR. This allowed us to detect exclusively end-labeled dsDNA molecules and unambiguously identify their origin by exciting solely a specific set of molecules at the absorption wavelength of its permanent end label. We present a fundamentally distinct separation and monitoring scheme for dsDNA analysis. In our approach, we launch simultaneously several laser wavelengths through a single WG, encode the fluorescence emitted by each set of end-labeled dsDNA molecules by exclusively time-modulating its excitation laser beam, and subsequently decode the emitted and detected fluorescence by Fourier analysis. In this manner we separate differently encoded fluorescence signals from each other, thus in principal enabling simultaneous multi-color interrogation of multiple sets of dsDNA fragments of different origin in a single small detection volume with a single ultrasensitive, albeit color-blind PMT.

As an example of a potential multiplex diagnostic application, we separated in a single experiment two diagnostically relevant samples obtained by multiplex ligation-dependent probe amplification (MLPA) - a set of 12 (origin: ERBB2 gene, $17^{\text {th }}$ human chromosome, associated with breast cancer) and a set of 23 (origin: RPS19 gene, $19^{\text {th }}$ human chromosome, associated with Diamond-Blackfan anemia) exclusively end-labeled dsDNA molecules sized 130-480 base-pairs [MRC Holland, web]. The results of this diagnostic experiment will be described in the following subsections.

\subsubsection{Multiplex ligation-dependent probe amplification (MLPA)}

Of the well-known PCR, MLPA [Schouten, 2002] is a recent high-resolution variation. It consists of optimized thermal cycles during which multiple, correlated primer probes parallely target multiple dsDNA sites, ligate in pairs 
and are amplified, resulting in multiple amplicon sets from different regions of a gene, each with its unique fluorescent label and comprising of multiple dsDNA fragments having different lengths. The pattern of peaks witnessed in the subject (patient-gene) electropherogram upon size-dependent MF CE separation of these amplicons must be compared to a reference (healthygene) electropherogram to draw accurate conclusions concerning the presence/absence of specific genetic mutations or chromosome aberrations in the region of the gene that was being targeted by the probes. In our experiments, we worked with commercially available MLPA samples [MRC Holland, web] which were further amplified in a conventional PCR by one of our industry partners within the EU project HIBISCUS [Hibiscus, web], namely Zebra Bioscience BV [Zebra, web] to reach a typical concentration of $10 \mathrm{nM}$ in order to detect the fluorescence emitted from end-labeled dsDNA molecules with our current set-up. Ligated MLPA probes (MRCHolland) were amplified with PCR readymix (P4600 from Sigma). Custom oligonucleotide SALSA primers (synthesized by Invitrogen) were modified at their two (5' and $3^{\prime}$ ) ends with Alexa fluor 488 or 647 . The PCR reaction mix used for amplification contained $400 \mathrm{nM}$ of labeled forward and reverse SALSA primers. In our specific example described in this chapter, 12 dsDNA molecules were MLPA-extracted from the ERBB2 gene (associated with breast cancer) on the $17^{\text {th }}$ human chromosome, and 23 dsDNA molecules were MLPA-extracted from the RPS19 gene (associated with Diamond-Blackfan anemia) on the $19^{\text {th }}$ human chromosome, as a sample mixture. Each probe set was exclusively fluorescently labeled during PCR amplification, the former with Alexa Fluor 488 and the latter with Alexa Fluor 647. A 3-step PCR protocol was employed for synthesis utilizing an iCycler (Bio-Rad). Final amplicons contained fluorescent species at both $3^{\prime}$ and $5^{\prime}$ ends of the dsDNA. In principle, MLPA-PCR multiplexing and exclusive fluorescent labeling can simultaneously be applied to probes from different regions of the same gene in a single reaction [Bunyan, 2007].

As a preliminary test in order to make sure that the sensitivity of the optofluidic approach is indeed sufficient for analyzing end-labeled MLPA samples, the 12-probe mixture was MF CE separated and fluorescently monitored in the presence, and then in the absence, of intercalating SYBR Green I dye molecules, based on the experimental protocol of chapter 3, section 3.3.1. The results are shown in Fig. 5.7.

The sizing accuracies of these diagnostically relevant MLPA probe dsDNA molecules amplified by PCR are comparable to the high values we achieved with the commercial dsDNA ladder molecules, as described in chapter 3. This is demonstrated in the calibration curves in Fig. 5.8. 

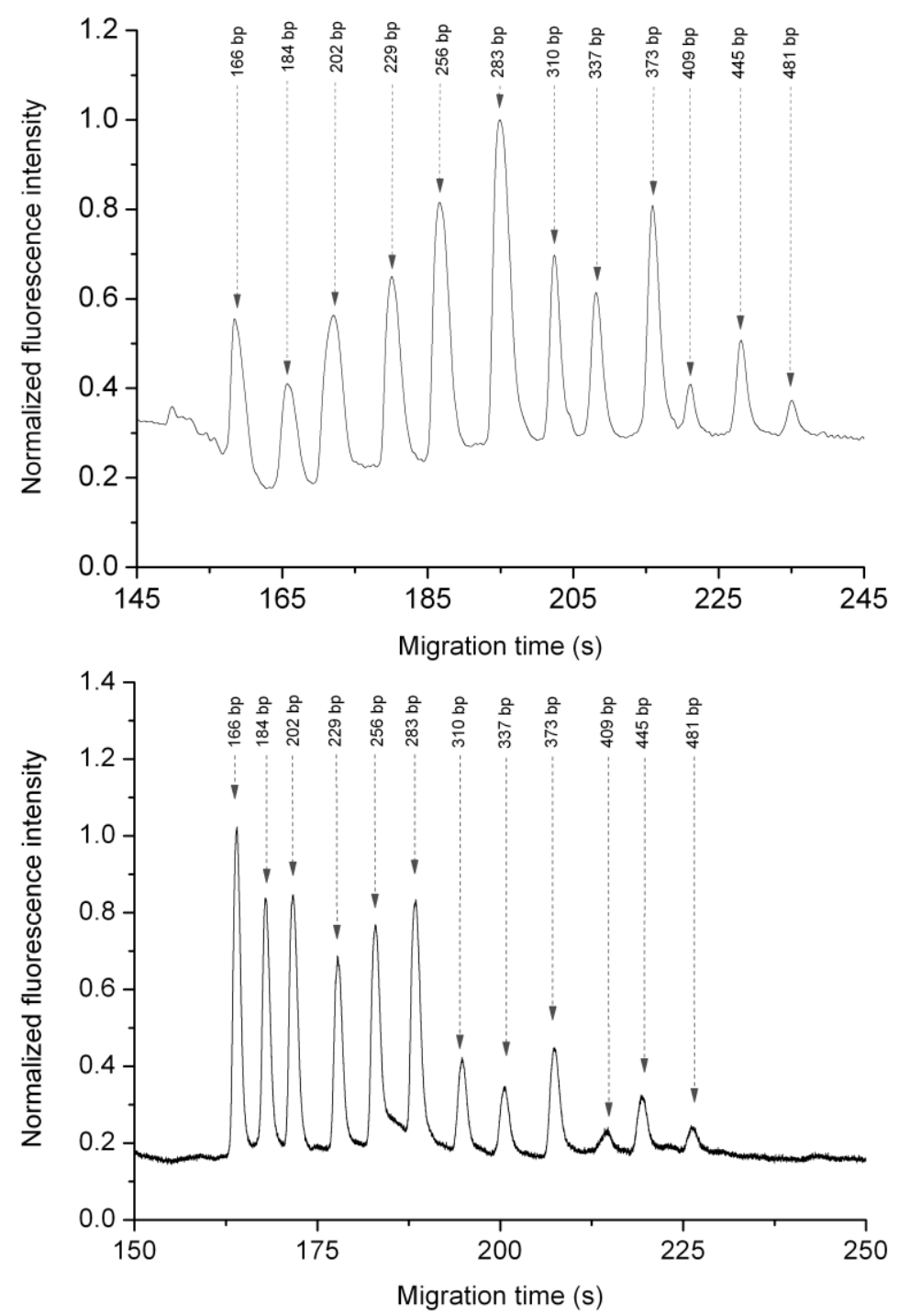

Fig. 5.7 Electropherograms depicting the separation of 12 MLPA probes from the ERBB-2 breast cancer gene detected by means of intercalating dye SYBR Green I (top), and covalent end-label Alexa Fluor 488 (bottom) 


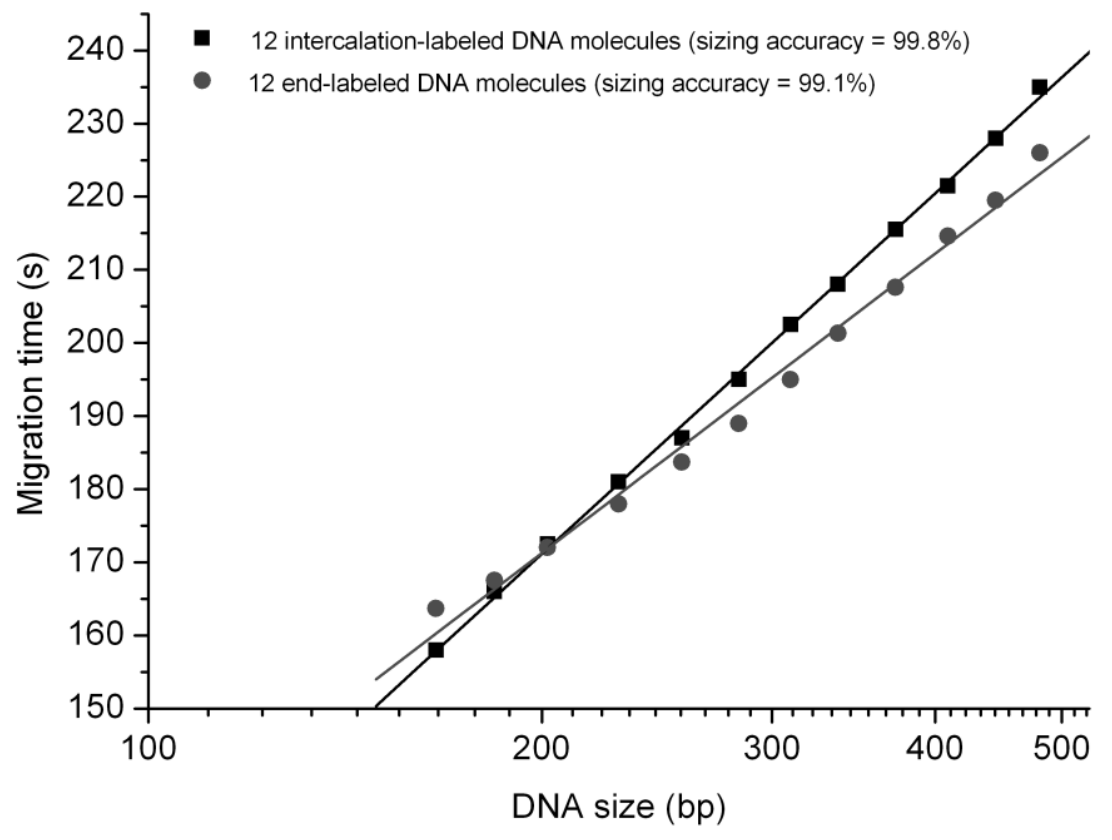

Fig. 5.8 Migration time vs. bp-size (on logarithmic scale) for the separation of 12 MLPA probe dsDNA molecules from the ERBB-2 breast cancer gene detected by means of intercalating dye SYBR Green I (black squares), and covalent end-label Alexa Fluor 488 (red circles) respectively

\subsubsection{Experimental protocol}

The same experimental protocol was implemented as the one described in chapter 3, section 3.3.1, except for the fact that in this case no intercalating dye was used for fluorescence labeling, since the molecules were a priori exclusively end-labeled. As described in section 5.4.1, MLPA probe dsDNA molecules were fluorescently covalently pre-end-labeled with exclusive dye molecules, and injected as a single mixture plug into the MF CE separation channel to be mutually separated and wavelength-selectively excited and detected.

\subsubsection{Fluorescence excitation and detection}

Two laser beams, the 488-nm line from an Ar-ion laser (mechanically amplitude-modulated at $17 \mathrm{~Hz}$ by means of a chopper) and the $635-\mathrm{nm}$ line from a red laser diode (electro-optically amplitude modulated at $31 \mathrm{~Hz}$ ) were combined by an external fiber combiner and simultaneously launched into the excitation WG. For fluorescence detection, a PMT (H7421-40, Hamamatsu Photonics K.K.) cooled down to $203 \mathrm{~K}$ was built onto the output 
port of an inverted microscope (DM5000, Leica Microsystems $\mathrm{GmbH}$ ) and aligned to collect the fluorescence signal through the DW toward the end of the MF CE separation channel, close to reservoir 4, where the plug separation is highest. Combination of excitation/emission band filters (K3, Leica Microsystems $\mathrm{GmbH}$, and XF57, Omega Optical, Inc.) in the fluorescence collection path ensured that only the fluorescence signals emitted by the fluorescently labeled dsDNA molecules reached the PMT, while all other sources of background signal, including the scattered laser excitation signals, were rejected.

\subsubsection{Experimental results}

The cumulative fluorescence signal as measured by the PMT is shown in Fig. 5.9 (top). With the two excitation lasers modulated with different, harmonically uncorrelated frequencies, Fourier analysis of the detected signal, based on the spectrum shown in Fig. 5.9 (bottom), resolves the spatiotemporally overlapping contributions at the two different wavelengths, thus separating similarly or even equally sized dsDNA molecules by identifying their origins via exciting their permanent end-labels with unique temporal signatures, as shown in Fig. 5.10.

These results indicate the potential of this method in multiplex genetic diagnostics, where samples from two independent genes, associated with two different illnesses have been analyzed simultaneously, nevertheless unambiguously. By treating one of the two samples (in this case the 12molecule mixture) as a "known" sample, the sizes of the molecules in the other sample (in this case the 23-molecule mixture), can be calibrated. In Fig. 5.11, we have plotted the a priori known bp-sizes of the MLPA probe dsDNA molecules (both the 12 and 23 molecule sets). Such a curve would need to be generated in the case of an unknown sample in order to use this approach in self-calibration of dsDNA molecular sizes in a single experimental run. 

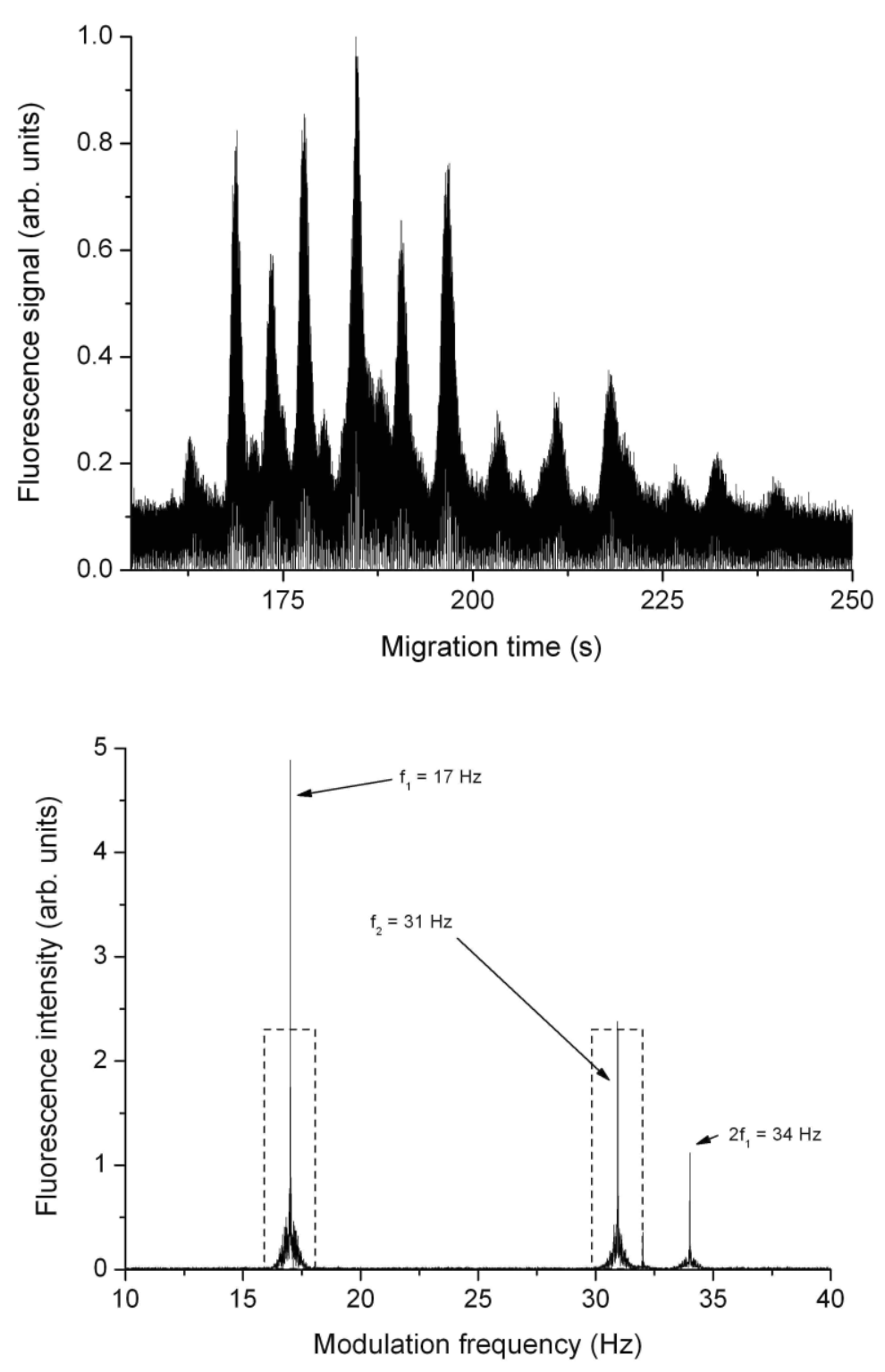

Fig. 5.9 (top) PMT-detected fluorescence signal from 35 end-labeled dsDNA molecules (consisting of $12 \& 23$ MLPA-PCR amplified dsDNA molecules from two chromosome regions) vs. migration time; (bottom) Fourier spectrum and applied transfer function (indicated by the dashed line) 

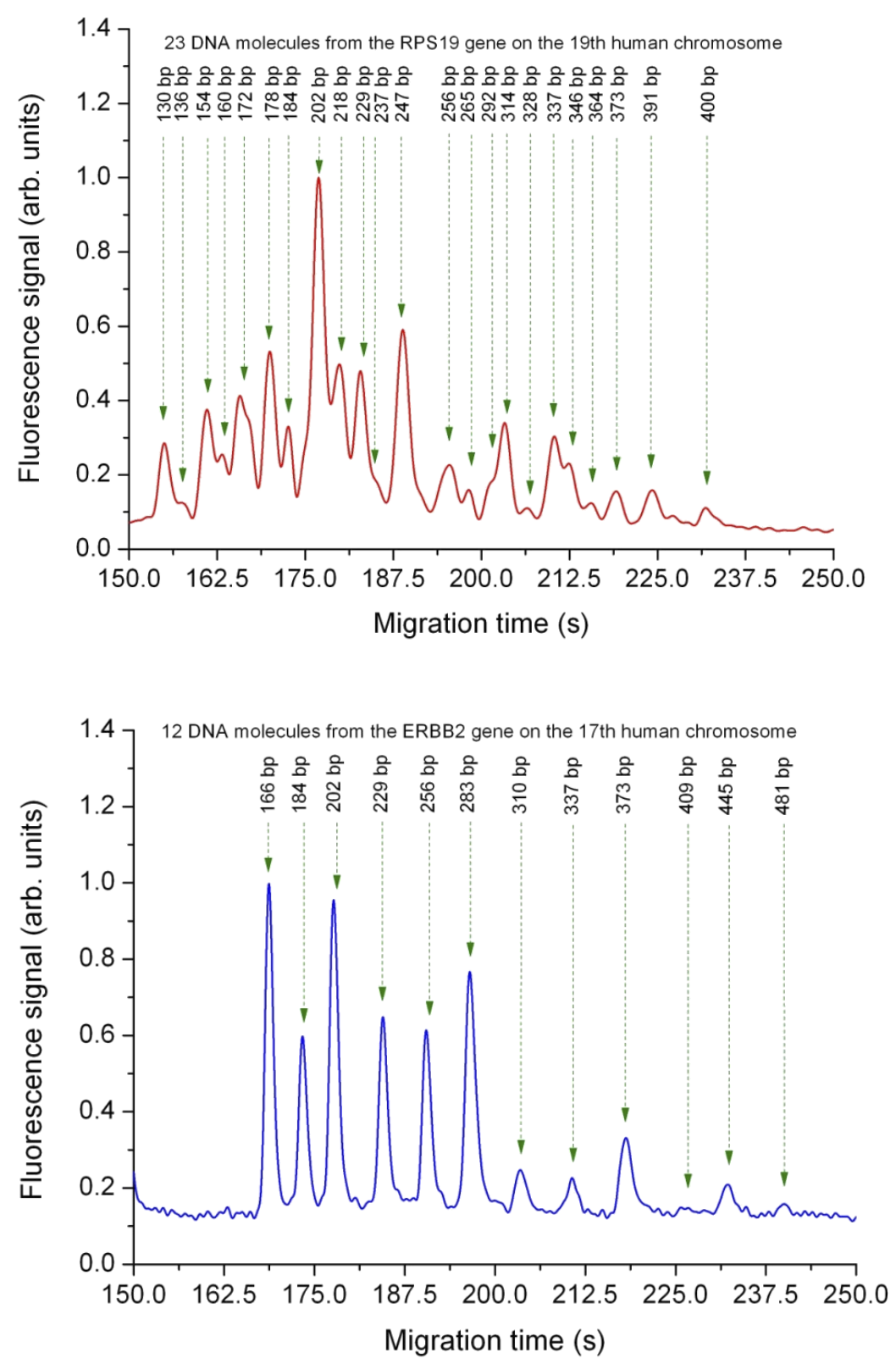

Fig. 5.10 individual signals separated by Fourier analysis of Fig.5.9; (top) 12 dsDNA molecules from a breast cancer gene and (bottom) 23 dsDNA molecules from a Diamond-Blackfan anemia gene. Several fluorescence peaks are below the noise level in Fig. 5.9, but are resolved in Fig. 5.10 by Fourier analysis. 


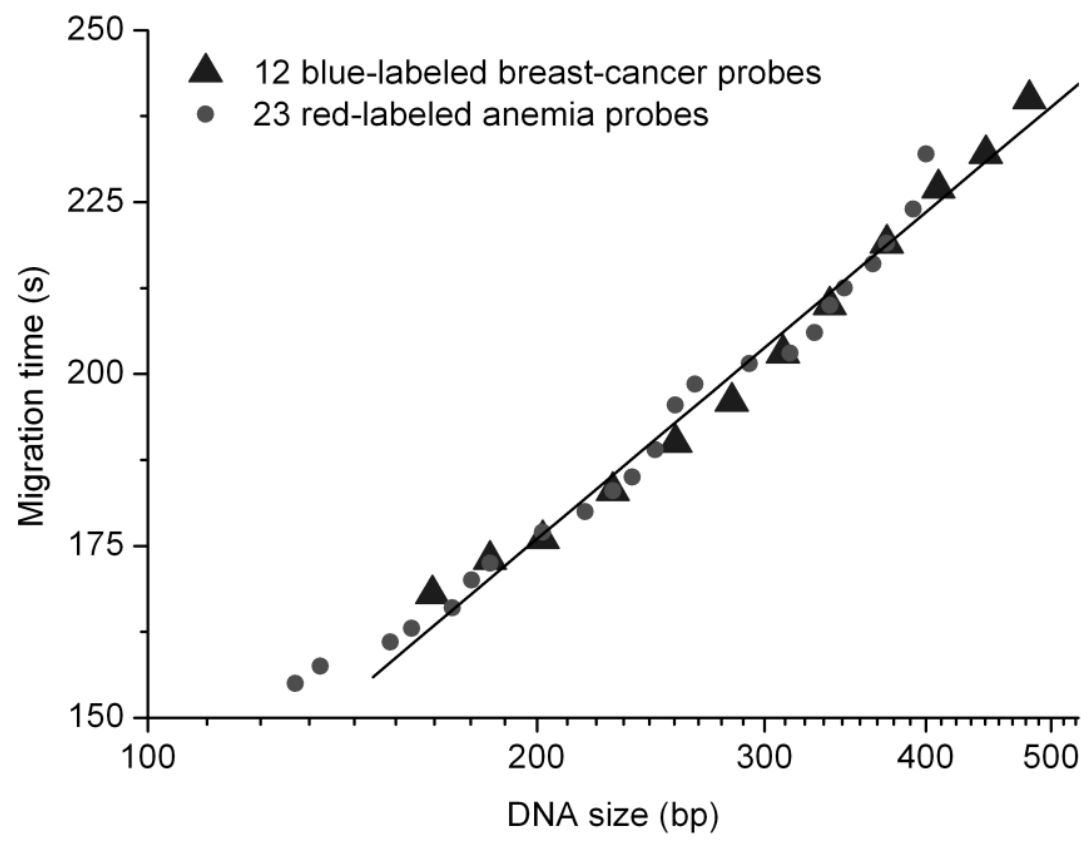

Fig. 5.11 A self-calibration curve for the simultaneous $M F C E$ separation analysis of the mixture of 12 breast cancer MLPA probes (blue triangles) and 23 anemia MLPA probes (red circles)

\subsubsection{Broad potential of the method}

The modulation-encoding and Fourier-decoding scheme described above enables exciting possibilities. In addition to the example described in the previous section, one can intentionally add a standard reference to a diagnostic dsDNA sample, thereby providing intrinsic molecule-size calibration to the set-up, making it independent of experimental parameters such as applied voltage, gel-matrix condition, and ambient temperature. Besides, one can also flow an unknown, potentially malign sample including dsDNA molecules of different sizes from a diagnostically relevant genomic region together with their healthy counterparts of otherwise identical molecules, all exclusively color labeled, thus providing unprecedented resolution to the experiment. In combination with MLPA, this identification scheme will potentially become a powerful tool. Multiple MLPA samples from different genomic regions, associated with predispositions to different diseases, each exclusively color-end-labeled - envisioned in Fig. 5.12 - can in principle be prepared in a single processing step [Bunyan, 2007]. 


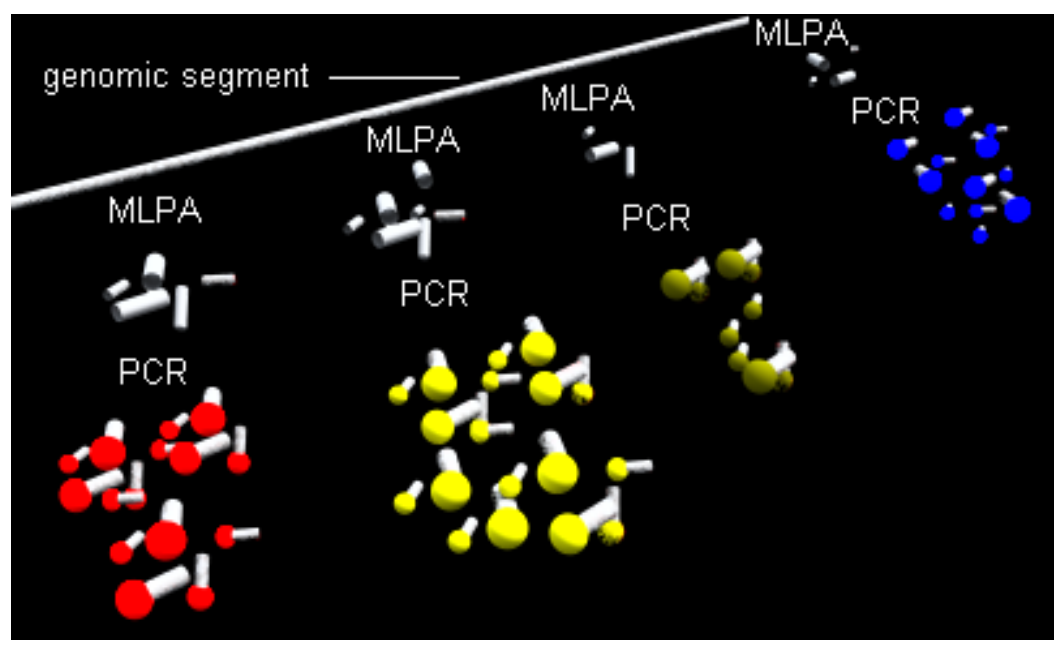

Fig. 5.12 MLPA extraction and exclusive permanent end-labeling during PCR of sets of dsDNA fragments from different gene regions

With the detection principle presented here, simultaneous investigation of several diagnostically relevant regions of the same or different genes becomes feasible in a single few-minute experiment, as partially illustrated in this section, ultimately leading to fast sampling of the complete genome for various defects. The results of Figs. 5.9 and 5.10 are an example of this principle (although in the specific experiment described in section 5.4.4, the two sets of dsDNA molecules were prepared in two separate MLPA experiments and then mixed).

This elegant approach introduces the concept of parallel processing to optical detection in LOCs. The experiment involves only two spectrally wellseparated wavelengths to avoid cross-excitation of overlapping absorption bands of two dyes by a single excitation wavelength. However, ongoing research toward the development and commercial mass-production of tunable, narrow-excitation-band [Walker, 2009] in standard II-VI material quantum-dot labels [Resch-Genger, 2008] can ultimately possibly give rise to much higher degrees of spectral multiplexing, thereby fully exploiting this extra dimension in on-chip biomolecule detection. The envisioned compact and portable dsDNA super-analyzers may address global public health challenges [Yager, 2006] cost-effectively, e.g. in cancer research by early identification of genomic deletions/insertions and in prenatal chromosomeaberration diagnosis, but also whenever time or sample-consumption efficiency is at stake; e.g., imagine an automated sampling robot collecting and analyzing samples on distant planets as pointers to the existence of life, a paleontologist decoding the DNA signature from a Jurassic fossil, or a 
virologist simultaneously analyzing multiple samples faster than the viruses mutate.

\subsection{Summary}

Integrated optical waveguide monitoring and high-resolution electrophoretic dsDNA separation were implemented at a low limit of detection, by employing modulation of excitation light sources. This enables the fluorescence excitation / detection of end-labeled dsDNA molecules at low concentrations. Exclusive modulation of the excitation signal and exclusive color labeling of the analyte dsDNA molecules lead to the possibility of multiplex fluorescent dsDNA analysis, owing to the selective excitation and detection of the concerned molecules, using a single, ultrasensitive but colorblind detector. For the purpose of comparison, another approach to multicolor fluorescent dsDNA analysis was described in this chapter, namely the dual-point, dual-wavelength approach. The advantages and disadvantages of this approach were discussed and it was found that the exclusive modulationbased approach is indeed the most generic, and universally applicable, and therefore is the preferred approach. 


\section{Conclusions and outlook}

This thesis started by introducing the field of on-chip integrated biochemical analyses and the consequent need for optical technologies that can also integrate vital sensing functionality in such labs-on-chips.

In the ensuing second chapter, our strategy of on-chip optofluidic integration was described. The microfluidic chips were fabricated by our partner LioniX BV in fused silica glass, whose material properties are well known to the potential end-users of the chip owing to its ubiquitous use in the world of biochemistry, enabling them to apply and optimize existing protocols at relative ease, as compared to a new material. The use of standard cleanroom-based, wafer-scale batch processing implies that the potential high-volume production of such chips in a commercial foundry would be feasible. The subsequent use of femtosecond laser waveguide writing, as implemented by our collaborators at the Politecnico di Milano, for optofluidic integration in the labs-on-chips, enables one to flexibly choose and locate the photonic circuitry one wishes to integrate, depending on the application. Such fast (few minutes per chip) post-processing thereby fully exploits the existing microfabrication technologies for the production of microfluidic chips, contributing a photonic added-value where appropriate. Optofluidic characterization of the chips confirmed their excellent functionality, and showed the feasibility of our setup to implement diagnostically relevant dsDNA separation/analyses. The optofluidic functionality enabled integrated WG fluorescent excitation during dsDNA analysis by means of electrophoretic separations, as described in the third chapter. High dsDNA sizing accuracies of up to $99 \%$ were achieved, and the potential role of integrated optics for a further 20 -fold enhancement in the separation resolution in future microfluidic chips was explored. The low limit of detection of 2.1 picomolar already provided a sensitivity higher than the values reported in the literature for other integrated optofluidic approaches for monitoring dynamic electrophoretic flow experiments.

While promising electrophoresis results with high sensitivities were described in the third chapter, they all shared a single limitation, namely the use of intercalating fluorescent dyes. These attach and detach themselves at numerous regular positions along an analyte dsDNA molecule, leading to a high relative fluorescence signal as compared to end-labeled molecules. However, due to the transitory nature of the attachment, the selective color coding (by corresponding selective fluorescent labeling) of specific sets of dsDNA probes would be rendered impossible. This would have been a setback in the attempt to construct a setup that could perform multiplex dsDNA analyses in a single electrophoretic experiment, with a unique color 
coding of the different sets of exclusively end-labeled dsDNA probes. As end-labeled dsDNA molecules provide a smaller fluorescence signal as compared to those intercalated with multiple dye molecules, it was therefore critical to enable the detection of low (as typically encountered in real diagnostic tests) concentrations of fluorescently end-labeled dsDNA molecules. The different steps toward such higher sensitivities were described in the fourth chapter. Specifically, the all-numerically implemented lock-in amplification delivered a 10-fold enhancement of the signal-to-noise ratio, leading to a limit of detection of 220 femtomolar. This improvement set the stage for the detection of end-labeled molecules, and made it realistically possible to perform the envisioned multi-color fluorescent dsDNA analysis.

Finally, the two key results described in the third and the fourth chapter were synergically integrated in the fifth and final chapter of the thesis. Our principle of parallel optical processing was based on color encoding by the unique modulation of multiple excitation laser beams to excite equally modulated fluorescence in the individual dsDNA probe sets, and the subsequent Fourier analytical decoding of the signal measured by a colorblind, but ultrasensitive photomultiplier tube. The multi-color signals corresponding to the individual dsDNA probe-sets - which in our example correspond to probes from anemia and breast cancer genes, respectively were thereby simultaneously, nevertheless unambiguously unraveled. This represents the key achievement of this thesis in demonstrating multi-color fluorescent dsDNA analysis in an optofluidic chip, a device capable of simultaneous multiplex dsDNA analysis based on color-coding of the fluorescence signals emitted by the analyte molecules, thanks to modulation of the excitation laser beams.

This approach introduces the concept of parallel processing to optical detection in LOCs, but can also be applied elsewhere. Our proof of principle involves only two spectrally well-separated wavelengths to avoid crossexcitation of overlapping absorption bands of two dyes by a single excitation wavelength. However, applying tunable narrow-band labels can give rise to much higher degrees of spectral multiplexing, thereby fully exploiting this extra dimension in on-chip biomolecule detection. The envisioned compact and portable dsDNA super-analyzers may address global public health challenges cost-effectively, e.g. in cancer research by early identification of genomic deletions/insertions and in prenatal chromosome-aberration diagnosis, but also whenever time or sample-consumption efficiency is at stake within possibly wide-ranging application examples in space research, paleontology, virology, etc, mentioned in chapter 5. Last but not least, two other aspects of the extension of this work in the future could be: 
(i) Integration of the optical detection on the chip, e.g. as a hybrid flip-chip - containing a microlens or even an integrated WG for collecting and focusing the fluorescence signal emitted from the microfluidic channels with a single electron-multiplying CCD or an avalanche photodiode for the sensitive detection of the fluorescence - that can be attached on top of the optofluidic chip.

(ii) Integration of the optical excitation on the chip, once again, e.g. as a hybrid flip-chip - containing solid-state light sources, such as LEDs, diode-lasers, or integrated optical waveguide lasers, e.g. based on rare-earth-ion (e.g. $\mathrm{Pr}^{3+}$ ) doping of $\mathrm{Al}_{2} \mathrm{O}_{3}$ - that can be attached to the side-facet of the optofluidic chip as to directly couple to the waveguides.

These developments will represent not only a significant advance in scientific terms, but also from a commercial, and a broader, societal point-ofview, whereby highly functional, point-of-care optofluidic devices would be deployed in the field, as "enlightened" biochemical labs-on-chips of the future. 


\section{List of abbreviations}

3D

AF

AFM

bp

CCD

$\mathrm{CE}$

$\mathrm{Cy}$

DNA

dsDNA

DW

EPDMA

EOF

fs

FWHM

HEC

HPC

LIF

LOC

LOD

MCE

$\mathrm{MF}$

MLPA

NA

nt

PCR

PMT

SNOM

SNR

ssDNA

WG
Three dimensional

Alexa Fluor

Atomic force microscopy

base pair

charge coupled device

Capillary electrophoresis

Cyanine

Deoxyribo nucleic acid

Double-stranded DNA

Detection window

Epoxy poly(dimethylacrylamide)

Electroosmotic flow

Femtosecond

Full width at half maximum

Hydroxyethyl cellulose

Hydroxypropyl cellulose

Laser induced fluorescence

Lab on a chip

Limit of detection

Microchip capillary electrophoresis

Microfluidic

Multiplex ligation-dependent probe amplification

Numerical aperture

Nucleotide

Polymerase chain reaction

Photomultiplier tube

Scanning near-field optical microscopy

Signal to noise ratio

Single-stranded DNA

Waveguide 


\section{References}

1. http://www3.appliedbiosystems.com/cms/groups/mcb marketing/doc uments/generaldocuments/cms 041990.pdf

2. http://www3.appliedbiosystems.com/AB_Home/applicationstechnolo gies/Real-TimePCR/TaqManvsSYBRGreenChemistries/index.htm

3. http://www.chem.agilent.com/en-us/products/instruments/lab-on-achip/2100bioanalyzer/Pages/default.aspx

4. https://www.beckmancoulter.com/eCatalog/CatalogItemDetails.do?pr oductId=12901

5. http://www.beckmancoulter.com/literature/Bioresearch/607397CEPrimer7.pdf

6. http://www.bio-rad.com

7. http://www.capilix.com

8. http://www.fisi.polimi.it/hibiscus

9. http://tools.invitrogen.com/content/sfs/manuals/cms 042179.pdf

10. http://www.iupac.org/goldbook/P04694.pdf

11. http://www.lionixbv.nl

12. http://www.mrc-holland.com

13. http://www.pacificbiosciences.com

14. http://www.roche-appliedscience.com/PROD_INF/BIOCHEMI/no4_05/pdf/p04.pdf

15. http://www.shimadzu.com

16. http://www.schott.com/lithotec/english/products/fused_silica/fused_s ilica.html 


\section{7. http://www.schott.com/lithotec}

18. http://www.targetdiscovery.com/ tdidocs/EOTrolTechGuideRevC.pd $\underline{\mathrm{f}}$

19. http://www.zebrabioscience.nl

20. Akbari, A., Albregtsen, F., and Lingjaerde, O.C., "Adaptive weighted least squares method for the estimation of DNA fragment lengths from agarose gels", Electrophoresis 23, 176-181 (2002).

21. Albarghouthi, M.N., and Barron, A.E., "Polymeric matrices for DNA sequencing by capillary electrophoresis", Electrophoresis 21, 40964111 (2000).

22. Altshuler, D., Daly, M.J., and Lander, E.S., "Genetic mapping of human disease", Science 322, 881-888 (2008).

23. Applegate Jr, R.W., Squier, J., Vestad, T., Oakey, J., Marr, D.W.M., Bado, P., Dugan, M.A., and Said, A.A., "Microfluidic sorting system based on optical waveguide integration and diode laser bar trapping", Lab Chip 6, 422-426 (2006).

24. Auroux, P.A., Reyes, D.R., Iossifidis, D., and Manz, A., "Micrototal analysis systems 2 . Analytical standard operations and applications," Anal. Chem. 74, 2637-2652 (2002).

25. Bello, M.S., Capelli, L., and Righetti, P.G., "Dependence of the electroosmotic mobility on the applied electric field and its reproducibility in capillary electrophoresis", J. Chromatogr. A 684, 311-322 (1994).

26. Bellouard, Y., Said, A., Dugan, M., and Bado, P., "Monolithic threedimensional integration of micro-fluidic channels and optical waveguides in fused silica," Mater. Res. Soc. Symp. Proc. 782, A3.2.1-A3.2.6 (2004).

27. Biver, T., de Biasi, A., Secco, F., Venturini, M., and Yarmoluk, S., "Cyanine dyes as intercalating agents: kinetic and thermodynamic studies on the DNA/Cyan40 and DNA/CCyan2 systems", Biophys. J. 89, 374-383 (2005).

28. Blair, D.P., and Sydenham, P.H., "Phase sensitive detection as a means to recover signals buried in noise", J. Phys. E 8, 621-627 (1975).

29. Bliss, C.L., McMullin, J.N., and Backhouse, C.J., "Rapid fabrication of a microfluidic device with integrated optical waveguides for DNA fragment analysis," Lab Chip 7, 1280-1287 (2007). 
30. Bliss, C.L., McMullin, J.N., and Backhouse, C.J., "Integrated wavelength-selective optical waveguides for microfluidic-based laser-induced fluorescence detection“, Lab Chip 8, 143-151 (2008).

31. Brody, J.R., and Kern, S.E., "History and principles of conductive media for standard DNA electrophoresis", Anal. Biochem. 333, 1-13 (2004).

32. Brown, L., Koerner, T., Hugh Horton, J., and Oleschuk, R.D., "Fabrication and characterization of poly(methylmethacrylate) microfluidic devices bonded using surface modifications and solvents," Lab Chip 6, 66-73 (2006).

33. Bruin, G.J.M., "Recent developments in electrokinetically driven analysis on microfabricated devices", Electrophoresis 21, 3931-3951 (2000).

34. Bunyan, D.J., Skinner, A.C., Ashton, E.J., Sillibourne, J., Brown, T., Collins, A.L., Cross, N.C., Harvey, J.F., and Robinson, D.O., "Simultaneous MLPA-based multiplex point mutation and deletion analysis of the Dystrophin gene", Mol. Biotechnol. 35, 135-140 (2007).

35. Crespi, A., Gu, Y., Ngamsom, B., Hoekstra, H.J.W.M., Dongre, C., Pollnau, M., Ramponi, R. van den Vlekkert, H.H., Watts, P., Cerullo, G., and Osellame, R., "Three-dimensional Mach-Zehnder interferometer in a microfluidic chip for spatially resolved label free detection", Lab Chip 10, 1167-1173 (2010).

36. Campbell, L.C., Wilkinson, M.J., Manz, A., Camilleri, P., and Humphreys, C.J., "Electrophoretic manipulation of single DNA molecules in nanofabricated capillaries", Lab Chip 4, 225-229 (2004).

37. Cran-McGreehin, S.J., Dholakia, K., and Krauss, T.F., "Monolithic integration of microfluidic channels and semiconductor lasers", Opt. Express 14, 7723-7730 (2006).

38. Ceriotti, L., Weible, K., de Rooij, N.F., and Verpoorte, E., "Rectangular channels for lab-on-a-chip applications", Microelectron. Eng. 67, 865-871 (2003).

39. Cerullo, G., Osellame, R., Taccheo, S., Marangoni, M., Polli, D., Ramponi, R., Laporta, P., and de Silvestri, S., "Femtosecond micromachining of symmetric waveguides at $1.5 \mu \mathrm{m}$ by astigmatic beam focusing", Opt. Lett., 27, 1938 (2002).

40. Chou, H.P., Spence, C., Scherer, A., and Quake, S.A., "A microfabricated device for sizing and sorting DNA molecules" Proc. Natl Acad. Sci. USA 96, 11 (1999).

41. Cleary, A., Garcia-Blanco, S., Glidle, A., Aitchison, J.S., Laybourn, P., and Cooper, J.M., "An integrated fluorescence array as a platform 
for lab-on-a-chip technology using multimode interference splitters," IEEE Sens. J. 5, 1315-1320 (2005).

42. Chiari, M., Damin, F., and Reijenga, J.C., "Characterization of poly(dimethylacrylamide) and the combination of poly(vinyl alcohol) and cetyltrimethylammonium bromide as dynamic electroosmotic flow suppression agents in capillary electrophoresis", J. Chromatogr. A 817, 15-23 (1998)

43. Chiari, M., Cretich, M., Damin, F., Cerotti, L., and Consonni, R, "New adsorbed coatings for capillary electrophroesis", Electrophoresis 21, 909-916 (2000).

44. Craighead, H.G., "Future lab-on-a-chip technologies for interrogating individual molecules" Nature 442, 387-393 (2006).

45. Cretich, M., Chiari, M., Rech, I., and Cova, S., "Use of highmolecular- mass polyacrylamides as matrices for microchip electrophoresis of DNA fragments", Electrophoresis 24, 3793-3799 (2003).

46. Cretich, M., Chiari, M., Pirri, G., and Crippa, A., "Electroosmotic flow suppression in capillary electrophoresis: chemisorptions of trimethoxy silane-modified polydimethylacrylamide", Electrophoresis 26, 1913-1919 (2005).

47. Daniel, D.C., Thompson, M., and Woodbury, N.W., "Fluorescence intensity fluctuations of individual labeled DNA fragments and a DNA binding protein in solution at the single molecule level: a comparison of photobleaching, diffusion and binding dynamics", J. Phys. Chem. B 104, 1382-1390 (2000).

48. Davis, K.M., Miura, K., Sugimoto, N., and Hirao, K., "Writing waveguides in glass with a femtosecond laser,' Opt. Lett. 21, 17291731 (1996).

49. Demana, T., Lanan, M., and Morris, M.D., "Improved separation of nucleic acids with analyte velocity modulation capillary electrophoresis“, Anal. Chem. 63, 2795-2797 (1991).

50. De Mello, A.J., "Control and detection of chemical reactions in microfluidic systems", Nature 442, 394-402 (2006).

51. Deutsch J.M., and Madden, T.L., "Theoretical studies of DNA during gel electrophoresis", J. Chem. Phys. 90, 2476-2486 (1989).

52. Dishinger, J.F., and Kennedy, R.T., "Serial immunoassays in parallel on a microfluidic chip for monitoring hormone secretion from living cells", Anal. Chem. 79, 947-954 (2007).

53. Dittrich, P.S., and Schwille, P., "An integrated microfluidic system for reaction, high-sensitivity detection, and sorting of fluorescent cells and particles", Anal. Chem. 75, 5767-5774 (2003). 
54. Dittrich, P.S., and Manz, A., "Single-molecule fluorescence detection in microfluidic channels - the Holy Grail in $\mu$ TAS?", Anal. Bioanal. Chem. 382, 1771-1782 (2005).

55. Dolnik, V., and Gurske, W.A., "Capillary electrophoresis in sieving matrices: selectivity per base, mobility slope, and inflection slope", Electrophoresis 20, 3373-3380 (1999).

56. Dumais, P., Callender, C.L., Noad, J.P., and Ledderhof, C.J., "Silicaon-silicon optical sensor based on integrated waveguides and microchannels," Photon. Technol. Lett. 17, 441-443 (2005).

57. Dumais, P., Callender, C.L., Ledderhof, C.J., and Noad, J.P., "Monolithic integration of microfluidic channels, liquid-core waveguides, and silica waveguides on silicon”, Appl. Opt. 45, 91829190 (2006).

58. Easley, C.J., Karlinsey, J.M., Bienvenue, J.M., Legendre, L.G., Roper, M.G., Feldman, S.H., Hughes, M.A., Hewlett, E.L., Merkel, T.J., Ferrance, J.P., and Landers, J.P., "A fully integrated microfluidic genetic analysis system with sample-in-answer-out capability", Proc. Natl Acad. Sci. USA 103, 19272-19277 (2006).

59. Eaton, S.M., Chen, S.W., Zhang, L., Zhang, H., Iyer, R., Aitchison, J.S., and Herman, P.R., "Telecom-band directional coupler written with femtosecond fiber laser," IEEE Photon. Technol. Lett. 18, 2174 2176 (2006).

60. Eid, J., Fehr, A., Gray, J., Luong, K., Lyle, J., Otto, G., Peluso, P., Rank, D., Baybayan, P., Bettman, B., Bibillo, A., Bjornson, K., Chaudhuri, B., Christians, F., Cicero, R., Clark, S., Dalal, R., Dewinter, A., Dixon, J., Foquet, M., Gaertner, A., Hardenbol, P., Heiner, C., Hester, K., Holden, D., Kearns, G., Kong, X., Kuse, R., Lacroix, Y., Lin, S., Lundquist., P., Ma, C., Marks, P., Maxham, M., Murphy, D., Park, I., Pham, T., Phillips, M., Roy, J., Sebra, R., Shen, G., Sorenson, J., Tomaney, A., Travers, K., Trulson, M., Vieceli, J., Wegener, J., Wu, D., Yang, A., Zaccarin, D., Zhao, P., Zhong, F., Korlach, J., and Turner, S., "Real-time DNA sequencing from single polymerase molecules", Science 323, 133-138 (2009).

61. Fleger, M., Siepe, D., and Neyer, A., "Microfabricated polymer analysis chip for optical detection", IEE Proc. Nanobiotechnol. 151, 159-161 (2004).

62. Foquet, M., Korlach, J., Zipfel, W., Webb, W.W., and Craighead, H.G., "DNA fragment sizing by single molecule detection in submicrometer-sized closed fluidic channels", Anal. Chem. 74, 14151422 (2002).

63. Fredlake, C.P., Hert, D.G., Kan, C.W., Chiesl, T.N., Root, B.E., Forster, R.E., and Barron, A.E., "Ultrafast DNA sequencing on a 
microchip by a hybrid separation mechanism that gives 600 bases in 6.5 minutes", Proc. Natl. Acad. Sci. USA 105, 476-481 (2008).

64. Friis, P., Hoppe, K., Leistiko, O., Mogensen, K.B., Hübner, J., and Kutter, J.P., "Monolithic integration of microfluidic channels and optical waveguides in silica on silicon", Appl. Opt. 40, 6246-6251 (2001).

65. Fruetel, J.A., Renzi, R.F., Vandernoot, V.A., Stamps, J., Horn, B.A., West, J.A.A., Ferko, S., Crocker, R., Bailey, C.G., Arnold, D., Wiedenman, B., Choi, W.Y., Yee, D., Shokair, I., Hasselbrink, E., Paul, P., Rakestraw, D., and Padgen, D., "Microchip separations of protein biotoxins using an integrated hand-held device", Electrophoresis 26, 1144-1154 (2005).

66. Fu. J.L., Fang, Q., Zhang, T., Jin, X.H., and Fang, Z.L., "Laser induced fluorescence detection system for microfluidic chips based on an orthogonal optical arrangement", Anal. Chem. 78, 3827-3834 (2006).

67. Fundador, E.V., Choudhary, D., Schenkman, J.B., and Rusling, J.F., "Accurate DNA fragment sizing by capillary electrophoresis with laser-induced fluorescence array for detection of sequence specificity of DNA damage", Anal. Chem. 80, 2212-2221 (2008).

68. Gao, Q., and Yeung, E.S., "A matrix for DNA separation: genotyping and sequencing using poly(vinylpyrrolidone) solution in uncoated capillaries", Anal. Chem. 70, 1382-1388 (1998).

69. Gas, B., and Kenndler, E., "Peak broadening in microchip electrophoresis: a discussion of the theoretical background", Electrophoresis 23, 3817-3826, 2002.

70. Gattass, R.R., and Mazur, E., "Femtosecond laser micromachining in transparent materials", Nature Photon 2, 219 (2008).

71. Ghosal, S., "Fluid mechanics of electroosmotic flow and its effect on band broadening in capillary electrophoresis", Electrophoresis 25, 214-228 (2004).

72. Glezer, E.N., Milosavljevic, M., Huang, L., Finlay, R.J., Her, T.H., Callan, J.P., and Mazur, E., "Three-dimensional optical storage inside transparent materials", Opt. Lett. 21, 2023-2025 (1996).

73. Goetz, S. and Karst, U., "Wavelength-resolved fluorescence detector for microchip capillary electrophoresis separations", Sens. Actuators B 123, 622-627 (2007).

74. Goetz, S. and Karst, U.,"Recent developments in optical detection methods for microchip separations", Anal. Bioanal. Chem. 387, 183192 (2007).

75. Gong, M., Wehmeyer, K.R., Limbach, P.R., Arias, F., and Heineman, W.R., "On-line sample preconcentration using field-amplified 
stacking injection in microchip capillary electrophoresis", Anal. Chem. 78, 3730-3737 (2006).

76. Gonzalez, M.G., Santiago, G.D., Slezak, V.B., and Peuriot, A.L., "Simple synchronic detection at audio frequencies through a PC sound card", Rev. Sci. Instrum. 78, 055108 (2007).

77. Guttman, A., Cohen, A.S., Heiger, D.N., and Karger, B.L., "Analytical and micropreparative ultrahigh resolution of oligonucleotides by polyacrylamide gel high-performance capillary electrophoresis", Anal. Chem. 62, 137-141 (1990).

78. Guttman, A., Cooke, N. J., "Effect of temperature on the separation of DNA restriction fragments in capillary gel electrophoresis", J. Chromatogr. 559, 267-283 (1991).

79. Han, J. and Craighead, H.G., "Separation of long DNA molecules in a microfabricated entropic trap array" Science 288, 1026 (2000).

80. Harrison, D.J., Fluri, K., Seiler, K., Fan, Z.H., Effenhauser, C.S., and Manz, A., "Micromachining a miniaturized capillary electrophoresis based chemical analysis system on a chip", Science 261, 895-897 (1993).

81. Heller, C., Pakleza, C., and Viovy J.L., "DNA separation with field inversion capillary electrophoresis", Electrophoresis 16, 1423-1428 (1995).

82. Herold, K., "Lab on a chip technology: fabrication and microfluidics", (Ed. A. Rasooly), Caister Academic Press (2009).

83. Herold, K., "Lab on a chip technology: biomolecular separation and analysis", (Ed. A. Rasooly), Caister Academic Press (2009).

84. Hjerten, S., and Srichaiyo, T., "Multi-point detection method for electrophoresis and chromatography in capillaries", US Patent 5114551 (1992).

85. Horlick, G., "Reduction of quantization effects by time averaging with added random noise", Anal. Chem. 47, 352-354 (1975).

86. Hovrath, J., and Dolnik, V., "Polymer wall coatings for capillary electrophoresis", Electrophoresis 22, 644-655 (2001).

87. Huang, X., Gordon, M.J., and Zare, R.N., "Current-monitoring method for measuring the electroosmotic flow rate in capillary zone electrophoresis", Anal. Chem. 60, 1837-1838 (1988).

88. Hubner, J., Mogensen, K.B., Jorgensen, A.M., Friis, P., Telleman P., and Kutter, J.P., "Integrated optical measurement system for fluorescence spectroscopy in microfluidic channels," Rev. Sci. Instrum. 72, 229-234 (2001).

89. Hunt, H.C., and Wilkinson, J.S., "Optofluidic integration for microanalysis", Microfluid Nanofluid 4, 53-79 (2008). 
90. Ishido, T., Ishikawa, M., and Hirano, K., "Analysis of supercoiled DNA by agarose gel electrophoresis using low-conducting sodium threonine medium", Anal. Biochem. 400, 148-150 (2010).

91. Iten, R., "Apparatus for emitting and detecting light in a nucleic acid amplification reaction," European Patent 1962084 (2007).

92. Itoh, K., Watanabe, W., Nolte, S., and Schaffer, C., "Ultrafast processes for bulk modification of transparent materials", MRS Bulletin 31, 620-625 (2006).

93. Jacobson, S.C., and Ramsey, J.M., "Microchip electrophoresis with sample stacking", Electrophoresis 16, 481-486 (2005).

94. Jakeway, S.C., de Mello, A.J., and Russell, E.L., "Miniaturized total analysis systems for biological analysis", Fresenius J. Anal. Chem. 366, 525-539 (2000).

95. Jiang, G., Attiya, S., Ocvirk, G., Lee, W.E., and Harrison, D.J., "Red diode laser induced fluorescence detection with a confocal microscope on a microchip for capillary electrophroesis", Biosens. Bioelectron. 14, 861 (2000).

96. Johnson, M.E., and Landers, J.P., "Fundamentals and practice for ultrasensitive laser-induced fluorescence detection in microanalytical systems", Electrophoresis 25, 3513-3527 (2004).

97. Jorgenson, J.W., and Lukacs, K.D., "Capillary zone electrophoresis", Science 222, 266-272 (1983).

98. Jung, H.J., and Bae, Y.C., "Theory for the capillary electrophoresis separation of DNA in polymer solutions", J. Chromatogr. A 967, 279-287 (2002).

99. Kalyakin, S.L., Sursyakova, V.V., Burmakina, G.V., and Rubailo, A.I., "Hydrodynamic suppression of the electroosmotic flow in capillary electrophroesis with indirect spectrophotometric detection", J. Anal. Chem. 64, 398-403 (2009).

100. Kaneta, T., Ueda, T., Hata, K., and Totarolmasaka, "Suppression of electroosmotic flow and its application to determination of electrophoretic mobilities in a poly (vinylpyrrolidone) coated capillary", J. Chromatogr. A 1106, 52-55 (2006).

101. Kang, S.H., Lee, S., and Yeung, E.S., “Atypical mobilities of single native DNA molecules in microchip electrophoresis revealed by differential interference contrast microscopy", Electrophoresis 27, 4149-4157 (2006).

102. Karger, A.E., Harris, J.M., and Gesteland, R.F., "Multiwavelength fluorescence detection for DNA sequencing using capillary electrophoresis", Nucleic Acids Res. 19, 4955-4962 (1991). 
103. Kim, Y., and Morris, M.D., "Pulsed-field capillary electrophoresis of multikilobase length nucleic acids in dilute methyl cellulose solutions", Anal. Chem. 66, 3081-3085 (1994).

104. Kim, Y., and Morris, M.D., "Separation of nucleic acids by capillary electrophoresis in cellulose solutions with mono- and bisintercalating dyes", Anal. Chem. 66, 1168-1174 (1994).

105. Kim, Y., and Yeung, E.S., "Separation of DNA sequencing fragments up to 1000 bases by using poly(ethylene oxide) filled capillary electrophoresis", J. Chromatogr. A 781, 315-325 (1997).

106. Krawczyk, M.J., Dulak, J., and Kulakowski, K., "Mean free path and peak dispersion in the geometration motion in gel electrophoresis," Electrophoresis 23, 182-185 (2002).

107. Kuswandi, B., Nuriman, Huskens, J., and Verboom, W., "Optical sensing systems for microfluidic devices: a review", Anal. Chim. Acta 601, 141 (2007).

108. Lagally, E.T., Emrich, C.A., and Mathies, R.A., "Fully integrated PCR-capillary electrophoresis microsystem for DNA analysis", Lab Chip 1, 102-107 (2001).

109. Lagally, E.T., and Mathies, R.A., "Integrated genetic analysis Microsystems”, J. Phys. D: Appl. Phys. 37, 245-261 (2004).

110. Lambeck, P.V., "Integrated optical sensors for the chemical domain", Meas. Sci. Technol. 17, 93-102 (2006).

111. Lander, E.S., et al, "Initial sequencing and analysis of the human genome", Nature 409, 860-921 (2001).

112. Landers, J.P., "Molecular diagnostics on electrophoretic microchips: a review", Anal. Chem. 75, 2919-2926 (2003).

113. Lee, G.B., Lin, C.H., and Guan, G.L., "Micro flow cytometers with buried SU-8_SOG optical waveguides," Sens. Actuators A 103, 165-170 (2003).

114. Leeds, A.R., van Keuren, E.T., Durst, M.E., Schneider, T.W., Currie, J.F., and Paranjape, M., "Integration of microfluidic and microoptical elements using a single-mask photolithographic step," Sens. Actuators A 115, 571-580 (2004).

115. Lei H.J., and Crothers, D.M., "Relaxation studies of the proflavine-DNA complex: the kinetics of an intercalation reaction", J. Mol. Biol. 39, 461-477 (1969).

116. Lerman, L.S., and Frisch, H.L, "Why does the electrophoretic mobility of DNA in gels vary with the length of the molecule?", Biopolymers 21, 995-997 (1982).

117. Levene, M.J., Korlach, J., Turner, S.W., Foquet, M., Craighead, H.G., and Webb, W.W., "Zero-mode waveguides for 
single-molecule analysis at high concentrations", Science 299, 682687 (2003).

118. Li, T., Tang, H., Luo, M., and Chen, G., "Single-cell analysis in a plastic microfluidic channel with a hadamard transform microscopic fluorescence imaging system", Anal. Lett. 37, 20532065 (2004).

119. Li, Z., Zhang, Z., Emery, T., Scherer, A., and Psaltis, D., "Single mode optofluidic distributed feedback dye laser", Opt. Express 14, 696-701 (2006).

120. Lien, V., Berdichevsky, Y., and Lo, Y.H., "A prealigned process of integrating optical waveguides with microfluidic devices," IEEE Photon. Technol. Lett. 16, 1525-1527 (2004).

121. Lien, V., Zhao, K., Berdichevsky, Y., and Lo, Y.H., "High sensitivity cytometric detection using fluidic-photonic integrated circuits with array waveguides," IEEE J. Sel. Top. Quantum Electron. 11, 827-834 (2005).

122. Lin, Y.C., "Design of low voltage driven capillary electrophoresis chips without moving electrical fields", Sens. Actuators B 80, 33-40 (2001).

123. Lin, Y.C., Ho, H.C., Tseng, K., and Hou, S.Q., "A polymethylmethacrylate electrophoresis microchip with sample preconcentrator", J. Micromech. Microeng. 11, 189-194 (2001).

124. Lin, C.H., Lee, G.B., Fu, L.M., and Chen, S.H., "Integrated optical fiber capillary electrophoresis microchips with novel spin on glass surface modification", Biosens. Bioelectron. 20, 83-90 (2004).

125. Lin, Y.W., and Chang, H.T., "Analysis of double-stranded DNA by capillary electrophoresis using poly(ethylene oxide) in the presence of hexadecyltrimethylammonium bromide", J. Chromatogr. A. 1130, 206-211 (2006).

126. Lin, C.H., Wang, J.H., and Fu, L.M., "Improving the separation efficiency of DNA biosamples in capillary electrophoresis microchips using high-voltage pulsed DC electric fields", Microfluid. Nanofluid. 5, 403-410 (2008).

127. Lin, S.W., Chang, G.L., and Lin, C.H., "Novel wavelengthresolved fluorescence detection for a high-throughput capillary electrophoresis system under a diascopic configuration", J. Chromatogr. A 1192, 198-201 (2008).

128. Liu, X., Du, D., and Mourou, G., "Laser ablation and micromachining with ultrashort laser pulses", IEEE J. Quantum Electron. 33, 1706-1716 (1997). 
129. Liu, C., Xu, X., Wang, Q., and Chen, J., "Mathematical model for DNA separation by capillary electrophoresis in entangled polymer solutions", J. Chromatogr. A 1142, 222-230 (2007).

130. Loughran, M., Cretich, M., Chiari, M., and Suzuki, H., "Separation of DNA in a versatile microchip", Sens. Actuators B 107, 975-979 (2005).

131. Lundqvist, L., Chiu, D.T., and Orwar, O., "Electrophoretic separation and confocal laser-induced fluorescence detection at ultralow concentrations in constricted fused silica capillaries", Electrophoresis 24, 1737-1744 (2003).

132. Mach, P., Dolinski, M., Baldwin, K.W., Rogers, J.A., Kerbage, C., Windeler, R.S., and Eggleton, B.J., "Tunable microfluidic optical fiber", Appl. Phys. Lett. 80, 4294-4296 (2002).

133. Marcinkevicius, A., Juodkazis, S., Watanabe, M., Miwa, M., Matsuo, S., and Misawa, H., "Femtosecond laser-assisted threedimensional microfabrication in silica", Opt. Lett. 26, 277-279 (2001).

134. Malcik, N., Ferrance, J.P., Landers, J.P., and Caglar, P., "The performance of a microchip based fiber optic detection technique for the determination of $\mathrm{Ca} 2+$ ions in urine", Sens. Actuators B 107, 2431 (2007).

135. Manz, A., Graber, N., and Widmer, H.M., "Miniaturized total chemical analysis systems: a novel concept for chemical sensing", Sens. Actuators B 1, 244-248 (1990).

136. Martinez Vazquez, R., Osellame, R., Nolli, D., Dongre, C., van den Vlekkert, H.H., Ramponi, R., Pollnau, M., and Cerullo, G., "Integration of femtosecond laser written optical waveguides in a labon-chip," Lab Chip 9, 91-96 (2009).

137. Martinez Vazquez, R., Osellame, R., Cretich, M., Chiari, M., Dongre, C., Hoekstra, H.J.W.M., Pollnau, M., van den Vlekkert, H.H., Ramponi, R., and Cerullo, G., "Optical sensing in microfluidic lab-on-a-chip by femtosecond-laser-written waveguides", Anal. Bioanal. Chem. 393, 1209-1216 (2009).

138. Martinez Vazquez, R., Osellame, R., Crespi, A., Dongre, C., Hoekstra, H.J.W.M., Pollnau, M., van den Vlekkert, H.H., van Weeghel, R., Watts, P., Ramponi, R., and Cerullo, G., "Threedimensional photonic devices fabricated by ultrafast lasers for optical sensing in lab-on-a-chip", Proceedings of the SPIE 7203, 720313 (2009).

139. Mazurczyk, R., Vieillard, J., Bouchard, A., Hannes, B., and Krawczyk, S., "A novel concept of the integrated fluorescence 
detection system and its application in a lab-on-a-chip microdevice", Sens. Actuators B 118, 11-19 (2006).

140. McDonald, J.C., Duffy, D.C., Anderson, J.R., Chiu, D.T., Wu, H., Schueller, O.J.A., and Whitesides, G.M., "Fabrication of microfluidic systems in poly(dimethylsiloxane)," Electrophoresis 21, 27-40 (1999).

141. Misiakos, K., Kakabakos, S.E., Petrou, P.S., and Ruf, H., “A monolithic silicon optoelectronic transducer as a real time affinity biosensor", Anal. Chem. 76, 1366-1373 (2004).

142. Miura, K., Qiu, J., Inouye, H., Mitsuyu, T., and Hirao, K., "Photowritten optical waveguides in various glasses with ultrashort pulse laser", Appl. Phys. Lett. 71, 3329-3331, (1997).

143. Mogensen, K.B., Friis, P., Hübner, J., Petersen, N., Jorgensen, A.M., Telleman, P., and Kutter, J.P., "Ultraviolet transparent silicon oxynitride waveguides for biochemical microsystems," Opt. Lett. 26, 716-718 (2001).

144. Mogensen, K.B., El-Ali, J., Wolff, A., and Kutter, J.P., "Integration of polymer waveguides for optical detection in microfabricated chemical analysis systems," Appl. Opt. 42, 40724079 (2003).

145. Mogensen, K.B., Klank, H., and Kutter, J.P., "Recent developments for detection in microfluidic systems", Electrophoresis 25, 3498-3512 (2004).

146. Monat, C., Domachuk, P., and Eggleton, B.J., "Integrated optofluidics: a new river of light", Nature Photon. 1, 106-114 (2007).

147. Myers F.B., and Lee, L.P., "Innovations in optical microfluidic technologies for point-of-care diagnostics", Lab Chip 8, 2015-2031 (2008).

148. Namasivayam, V., Lin, R., Johnson, B., Brahmasandra, S., Razzacki, Z., Burke, D.T., and Burns, M.A., "Advances in on-chip photodetection for applications in miniaturized genetic analysis systems", J. Micromech. Microeng. 14, 81-90 (2004).

149. Nolte, S., Will, M., Burghoff, J., and Tuennermann, A., "Femtosecond laser waveguide writing: a new avenue to threedimensional integrated optics", Appl. Phys. A 77, 109 (2003).

150. Novak, L., Neuzil, P., Pipper, J., Zhang, Y., and Lee, S., “An integrated fluorescence detection system for lab-on-a-chip applications", Lab Chip 7, 27-29 (2007).

151. Obeid, P.J., and Christopoulos, T.K., "Continuous flow DNA and RNA amplification chip combined with laser-induced fluorescence detection”, Anal. Chim. Acta 494, 1-9 (2003). 
152. Ogston, A.G., "The spaces in a uniform random suspension of fibers", Trans. Faraday Soc. 54, 1754-1757 (1958).

153. Okamura, Y., Yoshinaka, S., and Yamamoto, S., "Measuring mode propagation losses of integrated optical waveguides: a simple method," Appl. Opt. 22, 3892-3894 (1983).

154. Olivares, J.A., Stark, P.C., and Jackson, P., "Liquid core waveguides for full imaging of electrophoretic separations", Anal. Chem. 74, 2008-2013 (2002).

155. Olson, N.A., Khandurina, J., and Guttman, A., "DNA profiling by capillary array electrophoresis with non-covalent fluorescent labeling", J. Chromatogr. A 1051, 155-160 (2004).

156. Osellame, R., Taccheo, S., Marangoni, M., Ramponi, R., Laporta, P., Polli, D., de Silvestri, S., and Cerullo, G., "Femtosecond laser writing of active optical waveguides with astigmatically shaped beams," J. Opt. Soc. Am. B 20, 1559-1567 (2003).

157. Osellame, R., Chiodo, N., Della Valle, G., Taccheo, S., Ramponi, R., Cerullo, G., Killi, A., Morgner, U., Lederer, M., Kopf, D., "Optical waveguide writing with a diode-pumped femtosecond oscillator", Optics Letters 29, 1900-1902 (2004).

158. Osellame, R., Chiodo, N., Maselli, V., Yin, A., ZavelaniRossi, M., Cerullo, G., Laporta, P., Aiello, L., De Nicola, S., Ferraro, P., Finizio, A., and Pierattini, G., "Optical properties of waveguides written by a $26 \mathrm{MHz}$ stretched cavity Ti:sapphire femtosecond oscillator", Optics Express 13, 612-620 (2005).

159. Osellame, R., Chiodo, N., Della Valle, G., Cerullo, G., Ramponi, R., Laporta, P., Killi, A., Morgner, U., Svelto, O., "Waveguide lasers in the C-band fabricated by laser inscription with a compact femtosecond oscillator", J. Sel. Top. Quant. Electron. 12, 277285 (2006).

160. Osellame, R., Maselli, V., Martinez Vazquez, R., Ramponi, R., and Cerullo, G., "Integration of optical waveguides and microfluidic channels both fabricated by femtosecond laser irradiation," Appl. Phys. Lett. 90, 231118 (2007).

161. Paegel, B.M., Emrich, C.A., Wedemeyer, G.J., Scherer, J.R., and Mathies, R.A., "High throughput DNA sequencing with a microfabricated 96-lane capillary array electrophoresis bioprocessor", Proc. Natl Acad. Sci. USA 99, 574-579 (2002).

162. Pariat, Y.F., Berka, J., Heiger, D.N., Schmitt, T., Cohen, A.S., Foret, F., and Karger, B.L., "Separation of DNA fragments by capillary electrophoresis using replacable linear polyacrylamides", J. Chromatogr. 652, 57 (1993). 
163. Park, J., Lee, D., Kim, W., Horiike, S., Nishimoto, T., Lee, S.H., and Ahn, C.H., "Fully packed capillary electrochromatographic microchip with self-assembly colloidal silica beads", Anal. Chem. 79, 3214-3219 (2007).

164. Pile, D., "Eavesdropping on DNA replication", Nature Photon. 3, 79-80 (2009).

165. Psaltis, D., Quake, S.R., and Yang, C., "Developing optofluidic technology through the fusion of microfluidics and optics," Nature 442, 381-386 (2006).

166. Popelka, S., Kabatek, Z., Viovy, J.L., and Gas, B., "Peak dispersion due to geometration motion in gel electrophoresis of macromolecules", J. Chromatogr. A 838, 45-53 (1999).

167. Poterasu, M., "Time drift compensation of the electronic balance during the measurement of liquid crystal density", Meas. Sci. Technol. 19, 025102 (2008.)

168. Rech, I., Cova, S., Restelli, A., Ghioni, M., Chiari, M., and Cretich, M., "Microchips and single-photon avalanche diodes for DNA separation with high sensitivity", Electrophoresis 27, 37973804 (2006).

169. Reed, G.T., "Methods of measurement of passive integrated optical waveguides," Proc. IEE Colloquium on Measurements on Optical Devices, Digest No. 210, 21-27 (1992).

170. Resch-Genger, U., Grabolle, M., Cavaliere-Jaricot, S., Nitschke, R., and Nann, T., "Quantum dots versus organic dyes as fluorescent labels", Nat. Methods 5, 763-775 (2008).

171. Reyes, R.D., Iossifidis, D., Auroux, P.A., and Manz, A., "Micrototal analysis systems 1. Introduction, theory, and technology," Anal. Chem. 74, 2623-2636 (2002).

172. Rivera, L., Puyol, M., Villuendas, F., and Alonso, J., "Miniaturized setup for fluorescence sensing with optodes: characterization of a new hemicyanine ion-selective-based membrane", Sens. Actuators B 134, 863-868 (2008).

173. Rousseau, J., Drouin, G., and Slater, G.W., "Entropic trapping of DNA during gel electrophoresis: effect of field intensity and gel concentration", Phys. Rev. Lett. 79, 1945-1948 (1997).

174. Ruano, J.M., Benoit, V., Aitchison, J.S., and Cooper, J.M., "Flame hydrolysis deposition of glass on silicon for the integration of optical and microfluidic devices," Anal. Chem. 72, 1093-1097 (2000).

175. Saiki, R.K., Gelfand, D.H., Stoffel, S., Scharf, S.J., Higuchi, R., Horn, G.T., Mullis, K.B., and Erlich, H.A., "Primer directed 
enzymatic amplification of DNA with a thermostable DNA polymerase", Science 239, 487-491 (1988).

176. Salim, M., Wright, P.C., and McArthur, S.L., "Studies of electroosmotic flow and the effects of protein adsorption in plasmapolymerized microchannel surfaces", Electrophoresis 30, 1877-1887 (2009).

177. Salas-Solano, O., Carrilho, E., Kotler, L., Miller, A.W., Goetzinger, L., Sosic, Z., and Karger, B.L., "Routine DNA sequencing of 1000 bases in less than one hour by capillary electrophoresis with replacable linear polyacrylamide solutions", Anal. Chem. 70, 3996-4003 (1998).

178. Salieb-Beugelaar, G.B., Dorfman, K.D., van den Berg, A., and Eijckel, J.C.T., "Electrophoretic separation of DNA in gels and nanostructures", Lab Chip 9, 2508-2523 (2009).

179. Sanders, J.C., Breadmore, M.C., Kwok, Y.C., Horsman, K.M., and Landers, J.P., "Hydroxypropyl cellulose as an adsorptive coating sieving matrix for DNA separations: Artificial neural network optimization for microchip analysis", Anal. Chem. 75, 986-994 (2003).

180. Sartori, A., Barbier, V., and Viovy, J.L., "Sieving mechanisms in polymeric matrices", Electrophoresis 24, 421-440 (2003).

181. Schmidt, B.S., Yang, A.H., Erickson, D., and Lipson, M., "Optofluidic trapping and transport on solid core waveguides within a microfluidic device", Opt. Express 15, 14322 (2007).

182. Schmidt, O., Bassler, M., Kiesel, P., Knollenberg, C., and Johnson, N., "Fluorescence spectrometer-on-a-fluidic-chip", Lab Chip 7, 626-629 (2007).

183. Schouten, J.P., McElgunn, C.J., Waaijer, R., Zwijnenburg, D., Diepvens, F., and Pals, G., "Relative quantification of 40 nucleic acid sequences by multiplex ligation-dependent probe amplification", Nucleic Acids Res. 30, 57-69 (2002).

184. Schwarz, M.A. and Hauser, P.C., "Recent developments in detection methods for microfabricated analytical devices", Lab Chip 1, 1-6 (2001).

185. Scofield, J.H., "Frequency-domain description of a lock-in amplifier", Am. J. Phys. 62, 129-134 (1994).

186. Shen, F., Yu, Y., Yang, M., and Kang, Q., "Dual confocal laser induced fluorescence / movable contactless conductivity detector for capillary electrophoresis microchip", Microsyst. Technol. $15,881-885$ (2009). 
187. Siemsen K.J. and Bernard, J.E., "A Phase sensitive technique to measure small changes in laser frequency: application to measure the shift and broadening of a saturated absorption line of OsO4", Appl. Phys. B 81, 497-502 (2005).

188. Slater, G.W., "Theory of band broadening for DNA gel electrophoresis and sequencing", Electrophoresis 14, 1-7 (1993).

189. Slater, G.W., Mayer, P., and Grossman, P.D., "Diffusion, Joule heating, and band broadening in capillary gel electrophoresis of DNA", Electrophoresis 16, 75-83 (1995).

190. Slater, G.W., Guillouzic, S., Gauthier, M.G., Mercier, J.F., Kenward, M., McCormick, L.C., and Tessier, F., "Theory of DNA electrophoresis", Electrophoresis 23, 3791-3816 (2002).

191. Slater, G.W., Kenward, M., McCormick, L.C., and Gauthier, M.G., "The theory of DNA separation by capillary electrophoresis", Current Opinion Biotechnol. 14, 58-64 (2003).

192. Slater, G.W., Tessier, F., and Kopecka, K., "The electroosmotic flow (EOF): a review", Methods Mol. Biol. 583, 121134 (2010).

193. Soper, S.A., Flanagan, J.H., Legendre, B.L., Williams, D.C., and Hammer, D.N., "Near infrared laser induced fluorescence detection for DNA sequencing applications", J. Sel. Top. Quant. Electron. 2, 1129-1140 (1996).

194. Song, C., Nguyen, N.T., Tan, S.H., and Asundi, A.K., "Modelling and optimization of micro optofluidic lenses", Lab Chip 9, 1178-1184 (2009).

195. Srichaiyo, T., and Hjerten, S., "Simple multi-point detection method for high-performance capillary electrophoresis", J. Chromatogr. A 604, 85-89 (2001).

196. Stefanowicz-Hajduk, J., Filipowicz, M., Kosinksi, I., and Ochocka, J.R., "Capillary electrophoresis with dual laser detection in separation of amplified fragment length polymorphism fragments", J. Sep. Sci. 32, 3539-3543 (2009).

197. Sudor, J., and Novotny, M.V., "Separation of large DNA fragments by capillary electrophoresis under pulsed field conditions", Anal. Chem. 66, 2446-2450 (1994).

198. Sugino, H., Ozaki, K., Shirasaki, Y., Arakawa, T., Shoji, S., and Funatsu, T., "On-chip microfluidic sorting with fluorescence spectrum detection and multiway separation", Lab Chip 9, 1254-1260 (2009).

199. Sun, P. and Hartwick, R.A., "On-line kinetic monitoring for biochemical reactions using multi-point detection in high- 
performance capillary electrophoresis", J. Chromatogr. A 695, 279285 (1995).

200. Sun, Y., Kwok, Y.C., and Nguyen, N.T., "Modelling and experimental characterization of peak tailing in DNA gel electrophoresis", Microfluid. Nanofluid. 3, 323-332 (2007).

201. Temple, P.A., "An introduction to phase-sensitive amplifiers: an inexpensive student instrument", Am. J. Phys. 43, 801-807 (1975). 202. Terabe, S., Otsuka, K., and Ando, T., "Band broadening in electrokinetic chromatography with micellar solutions and opentubular capillaries", Anal. Chem., 61, 251-260 (1989).

203. Tian, H., and Landers, J.P., "Hydroxyethylcellulose as an effective polymer network for DNA analysis in uncoated glass microchips: optimization and application to mutation detection via heteroduplex analysis", Anal. Biochem. 309, 212-223 (2002).

204. Uchida, H., Zhang, W.Y., and Katsube, T., "High speed chemical image sensor with digital LAPS system", Sens. Actuators B 34, 446-449 (1996)

$205 . \quad$ Veerman, J.A., Otter, A.M., Kuipers, L., and van Hulst, N.F., "High definition aperture probes for near-field optical microscopy fabricated by focused ion beam milling," Appl. Phys. Lett. 72, 31153117 (1998).

206. Verpoorte, E., "Chip vision - optics for microchips," Lab Chip 3, 42-52 (2003).

207. Vezenov, D.V., Mayers, B.T., Conroy, R.S., Whitesides, G.M., Snee, P.T., Chan, Y., Nocera, D.G., and Bawendi, M.G., "A low-threshold, high-efficiency microfluidic waveguide laser", J. Am. Chem. Soc. 127, 8952-8953 (2005).

208. Vieillard, J., Mazurczyk, R., Morin, C., Hannes, B., Chevelot, Y., Desbene, P.L., Krawczyk, S., "Application of microfluidic chip with integrated optics for electrophoretic separations of proteins", J. Chromatogr. B 845, 218-225 (2007).

209. Viovy, J.L., "Electrophoresis of DNA and other polyelectrolytes: Physical mechanisms", Rev. Mod. Phys. 72, 813872 (2000).

210. Vogelgesang, J., "Limit of detection and limit of determination: application of different statistical approaches to an illustrative example of residue analysis", Fr. J. Anal. Chem. 328, 213220 (1987).

211. Von Bibra, M.L., and Roberts, A., "Refractive index reconstruction of graded-index buried channel waveguides from their mode intensities," J. Lightwave Technol. 15, 1695-1699 (1997). 
212. Walker, B.J., Nair, G.P., Marshall, L.F., Bulovic, V., and Bawendi, M.G., "Narrow-band absorption-enhanced quantum dot / Jaggregate conjugates", J. Am. Chem. Soc. 131, 9624-9625 (2009).

213. Wang, Y., Lin, Q., and Mukherjee, T., "A model for Joule heating induced dispersion in microchip electrophoresis", Lab Chip 4, 625-631 (2004).

214. Wang, Z., Sekulovic, A., Kutter, J.P., Bang, D.B., and Wolff, A., "Towards a portable microchip system with integrated thermal control and polymer waveguides for real-time PCR", Electrophoresis 27, 5051-5058 (2006).

215. Whitesides, G.M., "The origins and the future of microfluidics", Nature 442, 368-373 (2006).

216. Witek, M.A., Llopis, S.D., Wheatley, A., McCarley, R.L., and Soper, S.A., "Purification and preconcentration of genomic DNA from whole cell lysates using photoactivated polycarbonate (PPC) microfluidic chips", Nucleic Acids Res. 34, e74 (2006).

217. Wolfe, D.B., Vezenov, D.V., Mayers, B.T., Whitesides, G.M., Conroy, R.S., and Prentiss, M.G., "Diffusion controlled optical elements for optofluidics", Appl. Phys. Lett. 87, 181105 (2005).

218. Wolfson, R., "The lock-in amplifier: a student experiment", Am. J. Phys. 59, 569-572 (1991).

219. Yager, P., Edwards, T., Fu, E., Helton, K., Nelson, K., Tam, M.R., and Weigl, B.H., "Microfluidic diagnostic technologies for global public health", Nature 442, 381-393 (2006).

220. Yeung, K.C., and Lucy, C.A., "Suppression of electroosmotic flow and prevention of wall adsorption in capillary zone electrophoresis using zwitterionic surfactants", Anal. Chem. 69, 3435-3441 (1997).

221. Yin, D., Deamer, D.W., Schmidt, H., Barber, J.P., and Hawkins, A.R., "Single molecule detection sensitivity using planar integrated optics on a chip", Opt. Lett. 31, 2136-2138 (2006).

222. Yin, D., Lunt, E.J., Rudenko, M.I., Deamer, D.W., Hawkins, A.R., and Schmidt, H., "Planar optofluidic chip for single particle detection, manipulation, and analysis", Lab Chip 7, 1171-1175 (2007).

223. Zhang, L., Dang, F., and Baba, Y., "Microchip electrophoresis-based separation of DNA", J. Pharm. Biomed. Anal. 30, 1645-1654 (2003).

224. Zhang, H., Ho, S., Eaton, S.M., Li, J., and Herman, P.R., "Three-dimensional optical sensing network written in fused silica glass with femtosecond laser", Opt. Express 16, 14015-14023 (2008). 
225. Zhu, L., Stryjewski, W.J., and Soper, S.A., "Multiplexed fluorescence detection in microfabricated devices with both timeresolved and spectral-discrimination capabilities using near-infrared fluorescence", Anal. Biochem. 330, 206-218 (2004).

226. Zipper, H., Brunner, H., Bernhagen, J., and Vitzthum, F., "Investigations on DNA intercalation and surface binding by SYBR Green I, its structure determination and methodological implications", Nucleic Acids Res. 32, e103 (2004). 


\section{List of publications}

\section{Peer reviewed international journals}

1. C. Dongre, R. Dekker, H. J. W. M. Hoekstra, M. Pollnau, R. Martinez Vazquez, R. Osellame, G. Cerullo, R. Ramponi, R. van Weeghel, G. A. J. Besselink and H.H. van den Vlekkert, "Fluorescence monitoring of microchip capillary electrophoresis separation with monolithically integrated waveguides", Opt. Lett. $33,2503-2505$ (2008).

2. R. Martinez Vazquez, R. Osellame, M. Cretich, M. Chiari, C. Dongre, H.J.W.M. Hoekstra, M. Pollnau, H.H. van den Vlekkert, R. Ramponi and G. Cerullo, "Optical sensing in microfluidic lab-on-a-chip by femtosecond-laser-written waveguides", Anal. Bioanal. Chem. 393, 1209-1216 (2009).

3. (cover story) R. Martinez Vazquez, R. Osellame, D. Nolli, C. Dongre, H.H. van den Vlekkert, R. Ramponi, M. Pollnau and G. Cerullo, " Integration of femtosecond laser written optical waveguides in a lab-on-chip", Lab Chip 9, 91-96 (2009).

4. A. Crespi, Y. Gu, B. Ngamsom, H.J.W.M. Hoekstra, C. Dongre, M. Pollnau, R. Ramponi, H.H. van den Vlekkert, P. Watts, G. Cerullo, and R. Osellame, "Three-dimensional Mach-Zehnder interferometer in a microfluidic chip for spatially-resolved labelfree detection”, Lab Chip 10, 1167-1173 (2010).

5. C. Dongre, J. van Weerd, G.A.J. Besselink, R. van Weeghel, R. Martinez Vazquez, R. Osellame, G. Cerullo, M. Cretich, M. Chiari, H.J.W.M. Hoekstra, and M. Pollnau, "High-resolution electrophoretic separation and integrated-waveguide excitation of fluorescent DNA molecules in a lab on a chip", Electrophoresis 31, 2584-2588 (2010).

6. (invited review) R. Osellame, C. Dongre, R. Martinez Vazquez, R. Ramponi, H.J.W.M. Hoekstra, G. Cerullo, and M. Pollnau, "Femtosecond laser microstructuring: an enabling tool for optical sensing in labs-on-a-chip", submitted. 
7. C. Dongre, J. van Weerd, G.A.J. Besselink, R. Martinez Vazquez, R. Osellame, G. Cerullo, R. van Weeghel, H.H. van den Vlekkert, H.J.W.M. Hoekstra, and M. Pollnau, "Multi-color fluorescent DNA analysis in an optofluidic chip", submitted.

8. C. Dongre, J. van Weerd, R. van Weeghel, N. Bellini, R. Osellame, G. Cerullo, H.J.W.M. Hoekstra, and M. Pollnau, "Dual-point dualwavelength fluorescence monitoring of DNA separation in a lab on a chip", submitted.

9. C. Dongre, M. Pollnau, and H.J.W.M. Hoekstra, "All-numerical noise filtering of fluorescence signals in capillary electrophoresis", submitted.

\section{Conference proceedings}

10. R. Martinez Vazquez, R. Osellame, A. Crespi, C. Dongre, H.J.W.M. Hoekstra, M. Pollnau, H.H. van den Vlekkert, R. van Weeghel, P. Watts, R. Ramponi and G. Cerullo, "Threedimensional photonic devices fabricated by ultrafast lasers for optical sensing in lab-on-a-chip”, Proc. SPIE 7203, 720313 (2009).

\section{Book chapters}

11. (invited) C. Dongre, H.J.W.M. Hoekstra, and M. Pollnau, "Capillary electrophoresis and multi-color fluorescent DNA analysis in an optofluidic chip", In Fundamental Concepts, Practical Applications, and Limitations of Capillary Electrophoresis and Microchip Capillary Electrophoresis, Edited by C.D. Garcia and E. Carrilho, Wiley \& Sons (2011), submitted.

\section{Conference contributions}

12. C. Dongre, R. Dekker, H. J. W. M. Hoekstra, D. Nolli, R. Martinez-Vazquez, R. Osellame, P. Laporta, G. Cerullo, and M. Pollnau, "Characterization of femtosecond laser written waveguides for integrated biochemical sensing", Proceedings of the annual symposium of the IEEE LEOS - Benelux chapter, Brussels, Belgium, 2007, pp. 27-30 (Oral presentation). 
13. H. J. W. M. Hoekstra, R. Dekker, M. Dijkstra, C. Dongre, M. Hoekman, L. J. Kauppinen, P. V. Lambeck, M. Pollnau, and H. P. Uranus, "Integrated optics sensors for multi-sensing platforms", Proceedings CD-ROM of the STW Conference - The Sense of Contact, Zeist, The Netherlands, 2007 (Poster presentation).

14. C. Dongre, R. Dekker, H. J. W. M. Hoekstra, D. Nolli, R. Martinez-Vazquez, R. Osellame, P. Laporta, G. Cerullo, G. A. J. Besselink, R. van Weeghel and M. Pollnau, "Femtosecond laser written waveguides for fluorescence sensing during microchip capillary electrophoresis", Book of abstracts: $9^{\text {th }}$ European Conference on Optical Chemical Sensors and Biosensors (Europtrode), Dublin, Ireland, 2008, pp. 116-117 (Poster presentation).

15. C. Dongre, R. Dekker, H. J. W. M. Hoekstra, D. Nolli, R. Martinez-Vazquez, R. Osellame, P. Laporta, G. Cerullo, G. A. J. Besselink, R. van Weeghel and M. Pollnau, "Femtosecond laser written waveguides for fluorescence sensing during microchip capillary electrophoresis", Proceedings CD-ROM of the STW Conference - The Sense of Contact, Zeist, The Netherlands, 2008 (Poster presentation).

16. R. Osellame, R. Martinez-Vazquez, C. Dongre, R. Dekker, H. J. W. M. Hoekstra, R. Ramponi, M. Pollnau, and G. Cerullo, "Femtosecond laser fabrication for the integration of optical sensors in microfluidic lab-on-a-chip devices", Proceedings CDROM of the Conference on Ultrafast Phenomena, Stresa, Italy, 2008 (Oral presentation).

17. R. Osellame, R. Martinez-Vazquez, R. Ramponi, G. Cerullo, C. Dongre, R. Dekker, H. J. W. M. Hoekstra, and M. Pollnau, "Integrated optical sensing in a lab-on-a-chip by femtosecond laser written waveguides", Proceedings CD-ROM of the Conference on Lasers and Electro-Optics (CLEO), San José, California, USA, 2008 (Oral presentation). 
18. C. Dongre, R. Dekker, H. J. W. M. Hoekstra, D. Nolli, R. Martinez-Vazquez, R. Osellame, G. Cerullo, G. A. J. Besselink, and M. Pollnau, "Fluorescence sensing with femtosecond laser written waveguides in a capillary electrophoresis chip for monitoring molecular separation", Proceedings of the $14^{\text {th }}$ European Conference on Integrated Optics (ECIO), Eindhoven, The Netherlands, 2008, pp. 107-110 (Oral presentation, First prize - best paper award).

19. R. Osellame, R. Martinez Vazquez, R. Ramponi, G. Cerullo, C. Dongre, R. Dekker, H. J. W. M. Hoekstra, M. Pollnau, "Femtosecond laser microfabrication of optical waveguides in commercial microfluidic lab-on-a-chip", Proceedings CD-ROM of SPIE Photonics West, Conference 6886 "Microfluidics, BioMEMS, and Medical Microsystems VI", San José, USA, 2008 (Oral presentation).

20. C. Dongre, R. Dekker, H. J. W. M. Hoekstra, D. Nolli, R. Martinez-Vazquez, R. Osellame, G. Cerullo, G. A. J. Besselink, and M. Pollnau, "Monitoring fluorescently labelled DNA during capillary electrophoresis in a lab-on-a-chip with integrated femtosecond laser written waveguides", Proceedings CD-ROM of the Annual Meeting of the European Optical Society (Topical Meeting 1 - Biophotonics), Paris, France, 2008 (Oral presentation).

21. R. Martinez-Vazquez, D. Nolli, R. Osellame, C. Dongre, M. Pollnau, G. Cerullo, R. Ramponi, "Integration of femtosecond laser written waveguides for optical detection in microfluidic chips", Proceedings CD-ROM of the Annual Meeting of the European Optical Society (Topical Meeting 1 - Biophotonics), Paris, France, 2008 (Oral presentation).

22. C. Dongre, R. Dekker, H. J. W. M. Hoekstra, R. MartinezVazquez, R. Osellame, G. Cerullo, R. Ramponi, R. van Weeghel, G. A. J. Besselink, H.H. van den Vlekkert, and M. Pollnau, "Fluorescence monitoring of capillary electrophoresis separation in a lab-on-a-chip with monolithically integrated waveguides", Proceedings of the Annual Symposium of the IEEE LEOS Benelux Chapter, Enschede, The Netherlands, 2008, pp. 159-162 (Poster presentation). 
23. (invited) M. Pollnau, C. Dongre, R. Dekker, H. J. W. M. Hoekstra, R. Martinez-Vazquez, R. Osellame, G. Cerullo, R. Ramponi, R. van Weeghel, G. A. J. Besselink, H.H. van den Vlekkert, "Monitoring of DNA molecules in a lab on a chip with femtosecond laser written waveguides", Proceedings of the 39th Winter Colloquium on The Physics of Quantum Electronics Session "Medical and Bio Physics", Snowbird, Utah, USA, 2009, pp. 213 (Oral presentation).

24. R. Martinez Vazquez, R. Osellame, M. Cretich, C. Dongre, H.J.W.M. Hoekstra, H.H. van den Vlekkert, R. Ramponi, M. Pollnau, M. Chiari, and G. Cerullo, "Optical sensing in microchip capillary electrophoresis by femtosecond laser written waveguides", Proceedings of the 23rd International Symposium on Microscale Bioseparations, Boston, Massachusetts, USA, 2009, pp. 250 (Oral presentation).

25. C. Dongre, J. van Weerd, R. van Weeghel, R. Martinez Vazquez, R. Osellame, R. Ramponi, G. Cerullo, R. Dekker, G.A.J. Besselink, H.H. van den Vlekkert, H.J.W.M. Hoekstra, and M. Pollnau, "Integrated fluorescence sensing in a lab-on-a-chip for DNA analysis", Proceedings CD-ROM of the STW Conference - The Sense of Contact, Zeist, The Netherlands, 2009 (Poster presentation).

26. C. Dongre, R. Dekker, H. J. W. M. Hoekstra, R. Martinez Vazquez, R. Osellame, G. Cerullo, R. Ramponi, R. van Weeghel, G. A. J. Besselink and H.H. van den Vlekkert, and M. Pollnau "Fluorescence monitoring of capillary electrophoresis separation of biomolecules with monolithically integrated optical waveguides", Proceedings CD-ROM of the $3^{\text {rd }}$ Lab on a Chip European Congress, Stockholm, Sweden, 2009 (Poster presentation).

27. C. Dongre, R. Dekker, H. J. W. M. Hoekstra, R. Martinez Vazquez, R. Osellame, G. Cerullo, R. Ramponi, R. van Weeghel, G. A. J. Besselink and H.H. van den Vlekkert, and M. Pollnau "Fluorescence monitoring of microchip capillary electrophoresis separation with monolithically integrated waveguides", Technical Digest CD-ROM of the Conference on Lasers and Electro-Optics (CLEO), Baltimore, Maryland, USA, 2009 (Oral presentation). 
28. R. Martinez Vazquez, R. Osellame, M. Cretich, M. Chiari, C. Dongre, H.J.W.M. Hoekstra, M. Pollnau, H.H. van den Vlekkert, R. Ramponi and G. Cerullo, "Optical sensing by femtosecond laser written waveguides in a microfluidic chip for capillary electrophoresis ", Technical Digest CD-ROM of the Conference on Lasers and Electro-Optics (CLEO), Baltimore, Maryland, USA, 2009 (Oral presentation).

29. C. Dongre, J. van Weerd, R. van Weeghel, R. Martinez Vazquez, R. Osellame, R. Ramponi, G. Cerullo, R. Dekker, G.A.J. Besselink, H.H. van den Vlekkert, H.J.W.M. Hoekstra, and M. Pollnau, "Multi-point, Multi-wavelength Fluorescence Monitoring of DNA Separation in a Lab-on-a-chip with Monolithically Integrated Femtosecond-laser-written Waveguides", Technical Digest CDROM of the European Conference on Biomedical Optics (ECBO), Munich, Germany, 2009 (Oral presentation).

30. C. Dongre, J. van Weerd, G.A.J. Besselink, R. Martinez Vazquez, R. Osellame, R. Ramponi, G. Cerullo, R. van Weeghel, H.H. van den Vlekkert, H.J.W.M. Hoekstra, and M. Pollnau, "DNA separation and fluorescence monitoring by integrated waveguides in an optofluidic chip", Proceedings of the Annual Symposium of the IEEE Photonics Society - Benelux Chapter, Brussels, Belgium, 2009 (Oral presentation).

31. C. Dongre, J. van Weerd, G. A. J. Besselink, R. Martinez Vazquez, R. Osellame, G. Cerullo, R. van Weeghel, H.H. van den Vlekkert, H. J. W. M. Hoekstra, and M. Pollnau, "Multi-color fluorescent DNA analysis in an optofluidic chip", Conference on Lasers and Electro-Optics (CLEO), San Jose, CA, USA, 2010 (Oral presentation).

32. C. Dongre, M. Pollnau, and H.J.W.M. Hoekstra, "Modulationfrequency encoding/decoding for parallel detection in biophotonic sensing", Conference on Lasers and Electro-Optics (CLEO), San Jose, CA, USA, 2010 (Oral presentation). 
33. A. Crespi, Y. Gu, B. Ngamsom, H.J.W.M. Hoekstra, C. Dongre, M. Pollnau, R. Ramponi, H.H. van den Vlekkert, P. Watts, G. Cerullo, and R. Osellame, "Label-free detection in a lab-on-a-chip with a three-dimensional Mach-Zehnder interferometer", Conference on Lasers and Electro-Optics (CLEO), San Jose, CA, USA, 2010 (Oral presentation).

34. C. Dongre, J. van Weerd, G. A. J. Besselink, R. Martinez Vazquez, R. Osellame, G. Cerullo, R. van Weeghel, H.H. van den Vlekkert, H. J. W. M. Hoekstra, and M. Pollnau, "Multi-wavelength fluorescence sensing with integrated waveguides in an optofluidic chip", European Conference on Integrated Optics (ECIO), Cambridge, UK, 2010 (Oral presentation).

35. C. Dongre, J. van Weerd, R. van Weeghel, R. Martinez Vazquez, R. Osellame, G. Cerullo, G.A.J. Besselink, H.H. van den Vlekkert, H.J.W.M. Hoekstra, and M. Pollnau, "Multi-color fluorescent DNA analysis in an integrated optofluidic lab-on-a-chip", STW Conference - The Sense of Contact, Zeist, The Netherlands, 2010 (Poster presentation).

36. C. Dongre, H.J.W.M. Hoekstra, and M. Pollnau, "Electrophoretic separation and detection of a few DNA molecules in an optofluidic chip", Frontiers in Optics - Annual meeting of the Optical Society of America (OSA), Rochester, NY, USA, 2010 (Oral presentation).

37. C. Dongre, J. van Weerd, G.A.J. Besselink, R. Martinez Vazquez, R. Osellame, G. Cerullo, R. van Weeghel, H.H. van den Vlekkert, H.J.W.M. Hoekstra, and M. Pollnau, "Advances in fluorescent detection of molecules in an optofluidic chip", Annual meeting of the European Optical Society, Paris, France, 2010 (Oral presentation). 


\section{Samenvatting}

Het scheiden en sorteren van DNA moleculen, zoals ontwikkeld in de kader van het menselijk genoomproject, heeft het mogelijk gemaakt om verschillende ziektes genetisch in kaart te brengen. In dit proefschrift wordt onderzoek naar de mogelijkheden van een optofluidische chip besproken, waarbij de functies capillaire electroforese en laser-geïnduceerde fluorescentie op de chip geïntegreerd zijn. Excitatie van de gelabelde moleculen vindt hierbij plaats door licht in een optische golfgeleider die gefabriceerd is met femtoseconde-laser pulsen.

Geïntegreerde optica, voor de excitatie van fluorescentie, maakt een hoge ruimtelijke resolutie (12 $\mu \mathrm{m}$, doorsnede van de excitatiebundel) mogelijk in de electroforetische scheidingskanalen. Doordat deze bundeldiameter betrekkelijk klein is ten opzichte van de typische plugbreedte van een langskomende DNA plug $(\sim 250 \mu \mathrm{m})$ is een 20 -voudige verbetering van de resolutie in principe mogelijk indien men in staat zou zijn de electroforetische scheiding te verbeteren.

Tijdens de detectie van fluorescentie-gelabelde DNA moleculen, geëxciteerd via geïntegreerde optische golfgeleiders, bereikten we een nauwkeurigheid van $99 \%$ in het bepalen van de grootte van de DNA moleculen en een zeer hoge gevoeligheid. Een detectiegrens van 220 femtomolair is aangetoond, wat betekent dat de aanwezigheid van slechts 6 moleculen in de excitatievolume nog gedetecteerd kan worden.

Verder implementeerden wij het principe van parallele optische detectie in zo'n optofluidisch lab op een chip. Hierbij worden verschillende groepen van exclusief kleur-gelabelde DNA moleculen - die normaal gesproken niet onderscheidbaar zouden zijn, omdat ze ruimtelijk (in het scheidingskanaal) en qua detectietijd (in het electroferogram) samenvallen - toch optisch gescheiden. Het betreffende label is hierbij herkenbaar door het coderen van de excitatiebundel en het decoderen van het totale signal (via Fourier analyse), afkomstig van een kleurenblinde fotondetector. Als een demonstratie van bovengenoemd parallelle-detectie principe werden DNA monsters afkomstig van MLPA (engels: Multiplex Ligation-dependent Probe Amplification) van twee onafhankelijke genetische DNA-segementen, gerelateerd aan borstkanker en bloedarmoede, tegelijkertijd geanalyseerd.

De techniek voor het gelijktijdig en eenduidig analyseren van meerdere groepen van verschillend gelabelde biomoleculen, zoals in dit proefschrift beschreven, is zeer relevant voor een breed scala aan toepassingen in toekomstige lab-op-een-chip systemen. 


\section{Acknowledgements}

The past four years of doctoral research and life in Europe have been absolutely marvelous, and I owe my gratitude to many people for making this possible.

- First and foremost, my supervisor and promoter Prof. Dr. Markus Pollnau, and my daily supervisor and assistant-promoter Dr. Hugo Hoekstra, for giving me the opportunity to join the path of scientific research by joining the IOMS group as a PhD student on the EU project HIBISCUS four years ago, and for their extremely motivating presence that not only helped achieve wonderful results, but also helped me grow professionally into an independent researcher.

- My graduation committee: Prof. Dr. Christian Depeursinge, Prof. Dr. Vinod Subramaniam, Prof. Dr. Han Gardeniers, and Dr. Geert Besselink, for their valuable input, time and efforts to review this thesis.

- Jasper van Weerd, Geert Besselink, and Ronald Dekker for their vital contributions to the experiments.

- Anton Hollink, Henk van Wolferen, Meindert Dijkstra, Melis Jan Gilde, Theo Veenstra, and Albert Prak for their superb technical support and many fruitful discussions that helped optimize the experimental setup.

- Hans van den Vlekkert, René Heideman, and Rob van Weeghel for always providing their pragmatic industry perspective to the research.

- Giulio Cerullo and Roberto Osellame for the ambitious management of the EU project HIBISCUS, and Rebeca Martinez Vazquez, Nicola Bellini, Daniela Nolli, and Andrea Crespi for the femtosecond-laser waveguide writing, and for their amazing hospitality during my visits to Milano.

- Marina Cretich and Marcella Chiari for providing their "magic potion" coating for the microfluidic channel walls.

- Jan van Nieuwkasteele and Georgette Salieb-Beugelaar for fruitful discussions about the microfluidic setup and experiments. 
- Jincy Jose, Frans Segerink, Jeroen Korterik, Herman Offerhaus, Leo Chauvin, and Francois Parsy for their help in performing the waveguide cross-section refractive index measurements.

- Marcel Hoekman and Nur Ismail for sharing their amazing software skills.

- Joe Dragavon, Paul Watts, Matthias Pospiech, Moritz Simons, Guido Palmer, Uwe Morgner, Anne-Laure Calendron, Max Lederer, and Ron Suk for the many interesting multidisciplinary exchanges during the EU project meetings.

- Officemates: the omnipresent and omniscient Dimitri "de laserboer" my unofficial Dutch-integration consultant and swimming partner, Edward "the rugbyman" who taught me to speak Afrikaans, La Laura "the runner" for the "real" coffee, Ronald - whose sense of humor always made research (and conferences) highly enjoyable, and Gamar for ensuring a smooth landing during my first months in Enschede (and for the great Sudanese food).

- Colleagues: Nur - per tutte le serrate italiane, Marcel - for the many exchanges on Buddhism, Lasse - for his pleasant company during many "conferences", especially the one in Copenhagen (-), Imran, Feridun "Aabee" and Fehmi - for sharing with me Turkish culture (and food), So - for the discussions on Vipassana, Fei (company) for explaining the mysteries of the Chinese calender, Abu - who made sure that I never missed the spicy indian food, and Marko - who introduced me to the life on a Dutch farm.

- IOMS staff: Dr. Kerstin Wörhoff, Dr. René de Ridder, Prof. Dr. Alfred Driessen, Prof. Dr. Paul Lambeck, and Gabriel Sengo for the many inspiring talks and for their motivating presence.

- The IOMS secretaries Rita and Annitta for the always so smooth administration.

- Former colleagues: Gamar, Ronald, Wico, Jonathan, Christos, Edwin, Murali, Jing, Henry (Kelderman), Henri (Uranus), Mart, Dorleta, Amaia, and so on...for making my four years at IOMS a memorable learning experience. 
- ALL my friends in Enschede, in the Netherlands, and in Europe, who made absolutely sure that I NEVER felt homesick, even at Christmas!

- My parents for the value they placed on a good education, for (hopefully) not missing me too much the last four years, and for their constant encouragement from thousands of miles away (including the design of the front cover, and the Sanskrit phase on the back cover of this book).

- Et finalement, ma chère Audrey, merci d'être aussi patiente, pragmatique, positive, toujours raisonable, et pour tellement supporter et encourager ton "châtaigne" pendant des temps "un peu" stressants!

To all of you I owe this thesis! Thank you! 